\title{
Particle Image Velocimetry Measurements of an Airfoil-Vortex Interaction Event in a Two-Dimensional Wind Tunnel
}

\author{
by \\ Wesley M. Burwash, B.Sc. Engineering Physics
}

A Thesis submitted to

the Faculty of Graduate Studies and Research

in partial fulfilment of

the requirements for the degree of

Master of Applied Science

in Aerospace Engineering

Ottawa-Carleton Institute for

Mechanical and Aerospace Engineering

Department of Mechanical and Aerospace Engineering
Carleton University
Ottawa, Ontario, Canada

August 31, 2007

Copyright (C)

2007 - Wesley M. Burwash 


$\begin{array}{ll}\begin{array}{l}\text { Library and } \\ \text { Archives Canada }\end{array} & \begin{array}{l}\text { Bibliothèque et } \\ \text { Archives Canada }\end{array} \\ \begin{array}{l}\text { Published Heritage } \\ \text { Branch }\end{array} & \begin{array}{l}\text { Direction du } \\ \text { Patrimoine de l'édition }\end{array} \\ \begin{array}{l}\text { 395 Wellington Street } \\ \text { Ottawa ON K1A ON4 }\end{array} & \begin{array}{l}\text { 395, rue Wellington } \\ \text { Ottawa ON K1A ON4 } \\ \text { Canada }\end{array}\end{array}$

Your file Votre référence ISBN: 978-0-494-33640-3 Our file Notre référence ISBN: $978-0-494-33640-3$

NOTICE:

The author has granted a nonexclusive license allowing Library and Archives Canada to reproduce, publish, archive, preserve, conserve, communicate to the public by telecommunication or on the Internet, loan, distribute and sell theses worldwide, for commercial or noncommercial purposes, in microform, paper, electronic and/or any other formats.

The author retains copyright ownership and moral rights in this thesis. Neither the thesis nor substantial extracts from it may be printed or otherwise reproduced without the author's permission.
AVIS:

L'auteur a accordé une licence non exclusive permettant à la Bibliothèque et Archives Canada de reproduire, publier, archiver, sauvegarder, conserver, transmettre au public par télécommunication ou par l'Internet, prêter, distribuer et vendre des thèses partout dans le monde, à des fins commerciales ou autres, sur support microforme, papier, électronique et/ou autres formats.

L'auteur conserve la propriété du droit d'auteur et des droits moraux qui protège cette thèse. $\mathrm{Ni}$ la thèse ni des extraits substantiels de celle-ci ne doivent être imprimés ou autrement reproduits sans son autorisation.
In compliance with the Canadian

Privacy Act some supporting forms may have been removed from this thesis.

While these forms may be included in the document page count, their removal does not represent any loss of content from the thesis.
Conformément à la loi canadienne sur la protection de la vie privée, quelques formulaires secondaires ont été enlevés de cette thèse.

Bien que ces formulaires aient inclus dans la pagination, il n'y aura aucun contenu manquant.

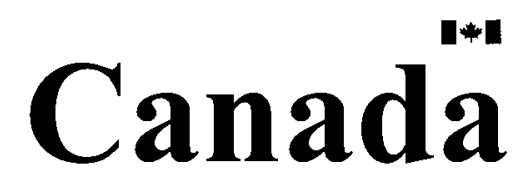




\section{Abstract}

The undesirable levels of noise and vibration generally associated with helicopters are, in much part, due to the rotor wake and related interactions of the trailing vorticity sheet with the rotor blades. These trailing vortices cause the rotor wake flow to be extremely complex, due to the large variations in the flow trajectory and intensities. Some insight into the rotor wake can be made using fluid flow measurements, enabling the validation of models used in computational fluid dynamics (CFD) simulations.

The flow complexity in the rotor wake makes a comprehensive fluid flow survey using point-measurement techniques such as hot-wire anemometry and laser Doppler velocimetry difficult, requiring thousands of individual measurements to resolve the flow. Particle image velocimetry (PIV) allows for the velocity measurements of entire two-dimensional flow fields to be acquired at once. This ability to measure the in-plane velocity of an entire flow field simultaneously makes PIV the obvious choice for this type of investigation, especially in the case where the vortex axis is parallel to the blade axis, effectively collapsing the flow phenomenon to two-dimensions.

Small olive oil droplets $\left(\rho=0.92 \mathrm{~g} / \mathrm{cm}^{3}\right)$, approximately $0.5 \mu \mathrm{m}$ in diameter, were used as fluid flow tracers and seeded downstream of the test section in a closed loop wind tunnel constructed with a pseudo two-dimensional test section. The tracers allow the PIV technique to 'see' how the flow is behaving, and the size and density of the seeding particles is of great importance when evaluating the accuracy of the measurements. In general, smaller seeding particles in large concentrations are ideal, however this comes at the cost of increased required laser illumination power such that a compromise must be made.

Two pulsed Nd:YAG lasers (Model: Gemini 15, New Wave Research, Inc, Fremont CA) 
with energies up to $120 \mathrm{~mJ} /$ pulse and a $15 \mathrm{~Hz}$ double-pulsing repetition rate were used to illuminate the two-dimensional plane (coplanar light sheets) around the test rotor. After selecting an adequate time delay between the pulses of each laser (depending on mean velocities and the image magnification), the flow following oil droplets were illuminated and a sequence of image pairs, each image being recorded in synchronization with the two laser illumination pulses, were obtained by a charge coupled device (CCD) camera $(1008 \times 1016$ pixels, 30 frames/sec). Reconstructing the two CCD camera images by using calibration parameters (distance from the camera to the light sheet and angle between the light sheet and the camera), the real displacement of sub-areas (interrogation areas) in the measurement plane can be obtained through statistical methods (cross-correlations). Knowing the time delay between pulses and the displacement vectors, an instantaneous twodimensional velocity vector map of the CCD camera's field of view in the laser illuminated plane was obtained. The $15 \mathrm{~Hz}$ repetition rate of each laser cavity allowed for a large number of fluid-flow measurements to be acquired during a short time period, of which were subsequently averaged (where applicable) to obtain a statistically valid steady-state velocity profile.

The test section included a stationary test rotor blade downstream of a vortex generating pitching airfoil (both NACA 0012, $\bar{c}=200 \mathrm{~mm}$ ). The vortex generating unit created a starting vortex at the inlet to the test section, which imitates the trailing vortex observed in rotorcraft parallel blade-vortex interactions (BVI). The vortex convects downstream to impinge onto the test rotor and a BVI measurement was achieved.

Through the use of a novel timing circuit, $16 \mathrm{~ms}$ long time laps measurements (long enough for the vortex to pass by the test rotor completely) of a vortex in free flow and a vortex impacting a rotor at a miss distance of $h=0.05 \bar{c}$ were measured. In addition, in order to showcase the capabilities of the PIV system within the two-dimensional wind tunnel, measurements of the flow velocities in the wake of the vortex generating blade were conducted, and from this lower-bound turbulence level estimations were made. Measurements of the well known flow about the NACA 0012 test rotor were also conducted giving a good base case for comparison. 
The two-dimensional wind tunnel, designed specifically for PIV measurements of BVI events, is now ready for comprehensive experiments to be made with BVI noise reduction as the objective. The PIV system has been fully installed along with a successful first design of a vortex generating unit. This, along with certain modifications to the wind tunnel to allow for tracer particle introduction, have allowed for a relatively inexpensive method to investigate the BVI phenomenon in rotorcraft.

Chapter 1 will introduce the main topics of this thesis. Chapter 2 will elaborate on the measurement method which is used, particle image velocimetry. In chapter 3 , the fluid flow phenomenon which this work is intended to enable the measurement of, will be described in full. Chapter 4 discusses the methods used to make the measurements as well as the modifications that were made to the wind tunnel to make the measurements possible. Chapter 5 displays the capabilities of the PIV system with respect to blade-vortex interaction measurements, among others, as installed in the wind tunnel. A conclusion chapter will follow to summarize the work. 
Dedicated to my Mom and Dad, for their love and support. 


\section{Acknowledgments}

I would like to acknowledge my Master's degree co-supervisors, Doctor Edgar Matida and Doctor Fred Nitzsche from the Department of Mechanical Engineering at Carleton University. I am forever grateful for the opportunity to work with them both on this project and also to work with equipment that is available to only a handful of researchers in Canada. I am also very appreciative for their financial support throughout my extended stay here in Ottawa.

I would like to thank Doctor Junjie Gu, Doctor Matthew Johnson and their respective research groups for including me in the bi-weekly research meetings to watch others present work and allowing me to present mine. This opportunity gave me the chance to fine tune the verbal delivery of my work and gather valuable comments and suggestions from those attending the seminars.

This work could not have been possible if not for the Mechanical and Aerospace Engineering office staff, Christie Egbert, Marlene Groves and Nancy Powell. Their hard work allowed my rushed application to pass through with ease, my research to take precedence over administrative tasks and the purchasing of equipment to be a much smoother process than it is at many other facilities, known from personal experience.

Also, I would like to thank the members of the Carleton University Mechanical Engineering Laboratory and Machine Shop, specifically Alex Proctor and Kevin Sangster for extensive machine shop advice and fabrication of the airfoils used in the wind tunnel, Gary Clements, Steve Truttman and Fred Barrett for reliable electronic knowledge and helpful suggestions.

I would also like to acknowledge my family, Rick, Pat, Gregory, Rebecca and Jenine 
Burwash for their support throughout both my undergraduate and graduate degrees. Last, but certainly not least, my girlfriend Whitney Exton, who put up with my extended absence with grace and provided me with invaluable encouragement and support throughout as well as lending her expertise in professional writing to aid with the editing of this document.

viii 


\section{Table of Contents}

Abstract $\quad$ iii

List of Tables $\quad$ xii

List of Figures $\quad$ xiii

Nomenclature $\quad$ xvi

1 Introduction and Motivation $\quad 1$

1.1 Blade-Vortex Interaction . . . . . . . . . . . . . . . . . . . . . . 1

1.2 Particle Image Velocimetry $\ldots \ldots \ldots \ldots \ldots$

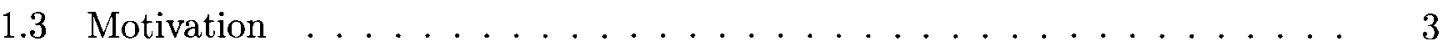

2 Particle Image Velocimetry 5

2.1 Tracer Particles . . . . . . . . . . . . . . . . . . . . 6

2.1.1 Traceability of Suspended Particles . . . . . . . . . . 8

2.1.2 Light Scattering Characteristics of Small Particles . . . . . . . . 10

2.1.3 Tracer Particle Generation and Delivery . . . . . . . . . . . . . . . 10

2.2 Laser Theory . . . . . . . . . . . . . . . . . . . . . . . 13

2.3 Charge-Coupled Device Camera Theory . . . . . . . . . . . . . . . . 18

2.4 Particle Image Velocimetry Image Processing . . . . . . . . . . . . . . 20

2.4 .1 Cross-Correlation . . . . . . . . . . . . . . . . . . 21

2.4 .2 Auto-Correlation . . . . . . . . . . . . . . . . 24

2.4.3 Peak Detection and Sub-Pixel Resolution Via Interpolation . . . . . 25 
2.4 .4 Fast Fourier Transform Correlation . . . . . . . . . . . . . . . 27

2.4.5 Window and Filter Functions to Compliment the FFT Cross-Correlation 30

2.4.6 Discrete Interrogation Window Overlap and Offset . . . . . . . . 32

2.5 Particle Image Velocimetry Image Post-Processing Methods . . . . . . . 33

2.5.1 Validation Techniques: Peak Validation . . . . . . . . . . 34

2.5.2 Validation Techniques: Range Validation . . . . . . . . . . 35

2.5.3 Validation Techniques: Moving Average . . . . . . . . . . . 35

2.5.4 Validation Techniques: Masking . . . . . . . . . . 36

2.5.5 Filtering: Average Filter . . . . . . . . . . . . . . 37

2.6 Accuracy Improving Methods for Particle Image Velocimetry . . . . . . . 37

2.6.1 Recursive Local-Correlation . . . . . . . . . . . . . . . . . 39

2.7 Particle Image Velocimetry Measurement Error Analysis . . . . . . . . . . 41

3 Blade (Airfoil)-Vortex Interaction $\quad 43$

3.1 Mechanics of the Blade-Vortex Interaction . . . . . . . . . . . . 47

3.2 Wind Tunnel Vortex Creation Methods . . . . . . . . . . . . . . 50

3.2 .1 Starting Vortex . . . . . . . . . . . . . . 52

3.2 .2 von Kármán Vortices . . . . . . . . . . . . . . . . . 54

3.3 Previous Study into the Blade-Vortex Interaction . . . . . . . . . . 55

3.3.1 Numerical Study of Blade (Airfoil)-Vortex Interaction . . . . . . . 56

3.3.2 Experimental Study of Blade (Airfoil)-Vortex Interaction . . . . . 58

4 Experimental Setup $\quad 64$

4.1 Wind Tunnel and Test Rotor . . . . . . . . . . . . . . . . 64

4.2 Vortex Creation . . . . . . . . . . . . . . . 67

4.2 .1 Timing ............................... 70

4.3 Particle Image Velocimetry Apparatus . . . . . . . . . . . . . . 73

4.3 .1 Tracer Particles . . . . . . . . . . . . . . 74

4.4 Test Conditions . . . . . . . . . . . . . . . . . . 75

4.4 Convergence Analysis $\ldots \ldots \ldots \ldots 76$ 
4.5 Data Analysis Procedure . . . . . . . . . . . . . . 77

4.5.1 Common Analysis Procedures . . . . . . . . . . . . . . 77

5 Results and Discussion $\quad 80$

5.1 Vortex Generator Wake with No Test Rotor Present . . . . . . . . . 80

5.2 Flow Field for the NACA 0012 Airfoil with no Vortex Impaction . . . . . . 82

5.3 Flow Field of a Free Vortex . . . . . . . . . . . . . . 85

5.4 Flow Field for the NACA 0012 Airfoil with Vortex Impaction . . . . . . . 88

5.5 Discussion $\ldots \ldots \ldots \ldots \ldots \ldots \ldots \ldots \ldots \ldots$

6 Conclusions $\quad 100$

$\begin{array}{ll}\text { List of References } & 102\end{array}$

Appendix A MatLab Post Processing Tools Code 107 


\section{List of Tables}

2.1 Common PIV tracer particles . . . . . . . . . . . . 7

2.2 Computational requirement comparison for FFT-CC and D-CC . . . . . . 28

3.1 Numerical vortex parameters . . . . . . . . . . . . . 57

5.1 Measurement, processing and post-processing parameters . . . . . . . 81 


\section{List of Figures}

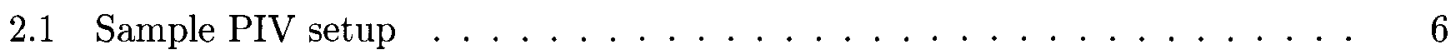

2.2 Tracer particle step velocity response . . . . . . . . . . . . . . 9

2.3 Relative tracer particle velocity . . . . . . . . . . . . . 9

2.4 Mie scattering versus viewing angle . . . . . . . . . . . . 11

2.5 Laskin nozzle aerosol generator . . . . . . . . . . . . . . . . . . 11

2.6 Simplified laser schematic . . . . . . . . . . . . . . . 14

2.7 Two state energy level diagram . . . . . . . . . . . . . . . . 14

$2.8 \mathrm{Nd}:$ YAG energy level diagram . . . . . . . . . . . . . . . 16

2.9 Q-switch schematic . . . . . . . . . . . . . . 18

2.10 CCD pixel model . . . . . . . . . . . . . . . . . . . . . . 19

2.11 Interline transfer CCD schematic . . . . . . . . . . . . . 20

2.12 Simplified PIV process . . . . . . . . . . . . . . . . 22

2.13 Spatial cross-correlation block diagram . . . . . . . . . . . . . . 23

2.14 FFT cross-correlation block diagram . . . . . . . . . . . . . 29

2.15 Top hat window function $\ldots \ldots \ldots \ldots \ldots \ldots$

2.16 Interrogation offset diagram $\ldots \ldots \ldots \ldots \ldots$

2.17 Raw vector peak validation $\ldots \ldots \ldots \ldots \ldots \ldots$

2.18 Super resolution PIV flowchart . . . . . . . . . . . . . 40

3.1 Tip vortex creation . . . . . . . . . . . . . . 44

3.2 BVI during descending flight $\ldots \ldots \ldots \ldots \ldots \ldots$

3.3 Flight path effects on BVI . . . . . . . . . . . 46

3.4 Phase emissions of BVI waves . . . . . . . . . . . . . . . 48 
3.5 Types of BVI in rotorcraft . . . . . . . . . . . . . . 49

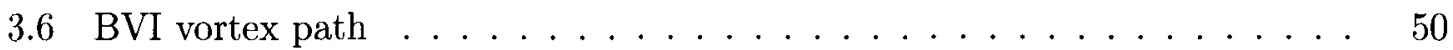

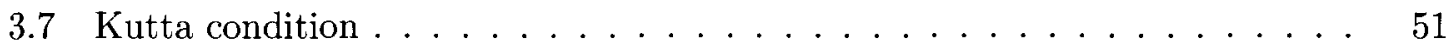

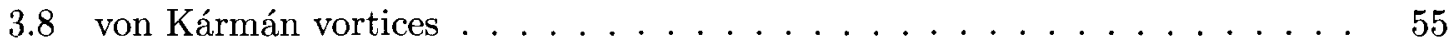

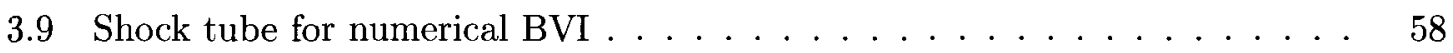

3.10 Experimental and numerical head-on BVI . . . . . . . . . . . 60

3.11 Schematic for rotor BVI test $\ldots \ldots \ldots \ldots$. . . . . . . . . 61

4.1 Wind tunnel side view schematic . . . . . . . . . . . . . 65

4.2 Wind tunnel test section schematic . . . . . . . . . . . . . 66

4.3 Centreline inlet and outlet velocity profiles . . . . . . . . . . . 67

4.4 Test section angularity measurements $\ldots \ldots \ldots \ldots \ldots$. . . . . . 68

4.5 Vortex generation blade . . . . . . . . . . . . . . . . 69

4.6 Vortex generation unit . . . . . . . . . . . . . 70

4.7 Timing circuits for vortex-PIV synchronization $\ldots \ldots \ldots 71$

4.8 Trigger IR beam in vortex generator $\ldots \ldots \ldots \ldots \ldots \ldots \ldots$

4.9 TTL voltage and angle of attack comparison $\ldots \ldots \ldots \ldots$. . . . . 72

4.10 PIV laser and camera mount . . . . . . . . . . . . . . . 73

4.11 Seeding schematic $\ldots \ldots \ldots \ldots \ldots \ldots \ldots$

4.12 Particle size distribution $\ldots \ldots \ldots \ldots \ldots$. . . . . . . . . . . . . .

4.13 Convergence analysis . . . . . . . . . . . . . . . . 78

5.1 Turbulence estimation $\ldots \ldots \ldots \ldots \ldots \ldots \ldots$

$5.220 \mathrm{~Hz}$ fan speed - NACA 0012 flow - coarse . . . . . . . . . . 84

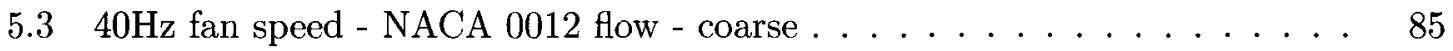

$5.460 \mathrm{~Hz}$ fan speed - NACA 0012 flow - coarse . . . . . . . . 86

$5.520 \mathrm{~Hz}$ fan speed - NACA 0012 flow - fine . . . . . . . . 86

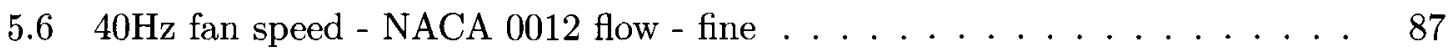

$5.760 \mathrm{~Hz}$ fan speed - NACA 0012 flow - fine . . . . . . . . 87

5.8 Vorticity - free vortex - set $1 \ldots \ldots \ldots \ldots \ldots$

5.9 Vorticity - free vortex $\ldots \ldots \ldots \ldots 2$ 
5.10 Vorticity - free vortex $-\operatorname{set} 2 \ldots \ldots \ldots \ldots \ldots$

5.11 Velocity - free vortex - set $3 \ldots \ldots \ldots \ldots$. . . . . . . . . 94

5.12 Vorticity - airfoil-vortex interaction - set $1 \ldots \ldots 95$

5.13 Vorticity - airfoil-vortex interaction - set $2 \ldots \ldots \ldots 6$

5.14 Vorticity - airfoil-vortex interaction - set $3 \ldots \ldots \ldots 7$

5.15 Velocity - airfoil-vortex interaction . . . . . . . . . . . . 98

5.16 Vorticity - close-up of airfoil-vortex interaction $\ldots \ldots \ldots$ 


\section{Nomenclature}

\section{Abbreviations}

Table N.1: $\quad$ Abbreviations

\begin{tabular}{ll}
\hline \hline AVI & Airfoil-Vortex Interaction \\
BJT & Bi-polar Junction Transistor \\
BVI & Blade-Vortex Interaction \\
CCD & Charge Coupled Device \\
CFD & Computational Fluid Dynamics \\
DPIV & Digital Particle Image Velocimetry \\
FFT & Fast Fourier Transform \\
LED & Light Emitting Diode \\
LDA & Laser Doppler Anemometry \\
NPN & Negative-Positive-Negative (a type of BJT) \\
NSS & Normalized Signal Strength \\
\hline
\end{tabular}

continued on next page... 
...continued from previous page

Table N.1: $\quad$ Abbreviations

RANS Reynolds Averaged Navier-Stokes

TTL Transistor-Transistor Logic

\section{Variable Notation}

\begin{tabular}{ll}
\hline Table N.2: & Variable Notation \\
\hline \hline$\alpha$ & Angle of attack \\
$a_{0}$ & Speed of sound \\
$\bar{c}$ & Rotor chord length \\
$C_{L}$ & Lift coefficient \\
$\left(\delta_{m}, \delta_{n}\right)$ & Interrogation region offset \\
$\Delta t$ & Time difference between two PIV images \\
$d_{c}$ & Cylinder diameter \\
$d_{p}$ & Tracer particle diameter \\
$\gamma$ & Angle between rotor blade axis and vortex axis \\
$h_{\mathrm{c}}$ & Circulation (vortex strength) \\
\hline & Maximum airfoil chamber
\end{tabular}

continued on next page...

xvii 
...continued from previous page

Table N.2: $\quad$ Variable Notation

\begin{tabular}{|c|c|}
\hline$I$ & Electrical current \\
\hline$k$ & $\begin{array}{l}\text { Generic particle image velocimetry image processing thresh- } \\
\text { old value (defined as necessary) }\end{array}$ \\
\hline$k_{e}$ & Turbulence kinetic energy \\
\hline$\Lambda$ & Light wavelength \\
\hline$L$ & Aerodynamic lift \\
\hline$\mu_{f}$ & Fluid viscosity (air) within the wind tunnel \\
\hline$(m, n)$ & Coordinates in the image plane (in pixels) \\
\hline$M_{t r}$ & Mach Trace Number in blade vortex interactions \\
\hline$N$ & General length of an interrogation window \\
\hline$\phi$ & The cross-correlation function \\
\hline$\psi$ & Rotor blade azimuth angle \\
\hline$Q$ & NPN transistor \\
\hline$\rho_{f}$ & Fluid density (air) within the wind tunnel \\
\hline$\rho_{p}$ & Tracer particle density \\
\hline$r_{b}$ & Radial position along rotor blade \\
\hline$R$ & Electrical Resistance \\
\hline$R e$ & Reynolds number, with respect to test rotor chord length \\
\hline
\end{tabular}

continued on next page...

xviii 
...continued from previous page

Table N.2: $\quad$ Variable Notation

\begin{tabular}{|c|c|}
\hline$R_{b}$ & Rotor blade radius \\
\hline$s_{n}, s_{m}$ & Displacement in the correlation plane \\
\hline$S$ & Scale factor between the object plane and image plane \\
\hline$S t$ & Stokes number \\
\hline$(u, v)$ & Coordinates in the spatial frequency domain \\
\hline $\mathbf{V}$ & Flow velocity vector \\
\hline$V_{+}$ & Positive rail voltage \\
\hline$V_{\infty}$ & Free stream velocity within the wind tunnel \\
\hline$V$ & Scalar velocity \\
\hline$\omega$ & Vortex shed frequency \\
\hline$\vec{\omega}$ & Vorticity \\
\hline$\Omega$ & Angular velocity of rotor blade \\
\hline$(x, y)$ & Coordinates in the object plane (in $\mathrm{mm}$ ) \\
\hline$x_{v}, y_{v}$ & Instantaneous vortex position, relative to test rotor nose \\
\hline$x_{t}, y_{t}$ & Position of the mounted test rotor \\
\hline$u^{\prime}, v^{\prime}$ & Fluctuations in the mean velocity \\
\hline$y_{b}$ & $\begin{array}{l}\text { Spacing between quarter chords of the test rotor and vortex } \\
\text { generating airfoils }\end{array}$ \\
\hline
\end{tabular}




\section{Chapter 1}

\section{Introduction and Motivation}

In this chapter the two main concepts involved with this study, blade-vortex interactions (BVI) and particle image velocimetry (PIV), will be introduced. The blade-vortex interaction is the flow field phenomenon which was investigated and particle image velocimetry was the chosen means by which to study the blade-vortex interaction. The motivation for the current work will also be discussed from the perspectives of advancement of the understanding of aerodynamical phenomena in rotorcraft and understanding of the method used to measure such phenomena, as well as future study, made possible by this work, to be done within a modified two-dimensional wind tunnel. Possible benefits that could arise from this and subsequent work will also be discussed briefly.

\subsection{Blade-Vortex Interaction}

The blade-vortex interaction is a rotorcraft-specific phenomenon in which the rotor blades of a helicopter interact aerodynamically with vortices that were shed from preceding rotor blades. This is an inescapable consequence due to the confined airspace that is available to the lifting bodies of a helicopter. During ascent, descent, hover and lateral flight (forward, reverse or sideways) the blades of a helicopter are traveling almost directly through the wake of all the blades on the aircraft, possibly including its own wake from a previous rotation. This creates a unique, complex and ultimately undesirable aerodynamic situation where the rotors are likely to encounter numerous fluid events that are difficult or impossible to predict 
in practice. Specifically, interactions during descent and sudden rotorcraft manoeuvres present the most significant noise and vibration production.

On any lifting wing (or blade) section of finite extent, the pressure differential between the upper and lower surfaces-the same differential which is responsible for the lifting forcecauses the undesired effect of wing (or blade) tip vortices. This phenomenon is the result of the higher pressure fluid seeping around the tip of the wing (or blade) to the upper surface in order to reach the lower pressure space. This curling motion around the tip of the lifting body causes localized vorticity, which is traced out as a vortex sheet since the lifting body is moving relative to the fluid (as it must be, to be generating the pressure gradient).

This problem is commonly observed with fixed wing aircraft for which small vertical wing sections, called winglets, have been designed and added to the wing tip in order to reduce the vortex effect. The benefit for fixed wing aircraft is a reduction of parasitic drag at the wing tip and thus effectively increasing the aspect ratio of the wing with no additional wingspan. However, this issue does not concern the effect of the shed vortices downstream of the aircraft, the effect which is responsible for BVI. Due to the rotational nature of the helicopter plus the addition of lateral motion, winglets are difficult to implement; thus, this solution cannot be implemented in rotorcraft as easily as they are for fixed wing aircraft.

\subsection{Particle Image Velocimetry}

Particle image velocimetry is a non-intrusive fluid measurement method which is capable of capturing the velocity of all points in an entire flow field simultaneously. Advanced PIV systems have the ability to measure three-dimensional flow fields through the use of two cameras as opposed to one camera for the two-dimensional velocity measurements. During this study, only the two-dimensional measurement technique was used due to the flow conditions at the measurement area and the equipment that was available.

The PIV velocity measurement is accomplished by illuminating a two-dimensional flow field with a powerful pulsed laser equipped with a light-sheet-forming cylindrical lens and then capturing an image of the laser light scattered by tracer particles (1-25 $\mu \mathrm{m}$ for gas 
flows and 10-100 $\mu \mathrm{m}$ for liquid flows) which are entrained in the flow. Generally, lasers with power averaging in the megawatt range over a short pulse, which is usually less than $10 \mathrm{~ns}$, are used. The image is taken with a special charge coupled device (CCD) camera at a near-perpendicular angle to both the flow direction and laser sheet. This single illuminationimaging process is repeated immediately afterwards (5-100 $\mu$ s delay, depending upon the flow velocity, field of view and desired post processing resolution).

The two images of the illuminated tracer particles, the second taken a very short period of time after the first, are broken into smaller interrogation regions, each of which are then compared to each other in a correlation process. The correlation is essentially a twodimensional convolution of the two interrogation region image intensity profiles with one correlation for each pair of interrogation regions (one in each captured image). This process returns a grid of velocity vectors, each vector describing the direction and speed of the tracer particles at that grid point which, with small enough tracer particles, estimates the kinematics of the flow in that region. The number of grid points, or grid resolution, depends upon the size of the image and the size of the chosen interrogation region, resulting in one vector for each rectangular interrogation region. The chosen size of these regions depends on the flow conditions and the selected delay between first and second exposures.

A balance must be found between tracer particle size and illumination by the laser. Smaller particles will trace the flow with much higher accuracy but require a much more powerful laser to be illuminated. A review of the particle image velocimetry measurement method was completed by Grant (1997) [1] and a comprehensive but practical guide written by Raffel et. al (1998) [2], together which provide a complete overview of the PIV measurement technique.

\subsection{Motivation}

The blade-vortex interaction in rotorcraft has been well-studied and is an area of continued investigation both experimentally (with PIV [3,4], laser doppler anemometry (LDA) [5] and other various techniques) and numerically (with computational fluid dynamics (CFD) $[5,6]$ 
and other numerical schemes [7]). Since BVI is an important source of both noise and vibration in helicopters, its reduction is of great interest for the future design of rotorcraft and also will extend the current understanding of noise and fatigue generation. The reduction of the undesirable noise caused by these interactions is important for both commercial applications, due to noise pollution laws in many major cities, general public acceptance and military applications, due to the obvious stealth advantages.

The goal of this study is to further develop a pre-existing two-dimensional wind tunnel to the point where investigations can be performed regarding the issue of blade-vortex interactions in rotorcraft. It will be shown that the wind tunnel, equipped with a powerful PIV system, is capable of observing the creation of a vortex and its ensuing collision with a fixed section of a rotor blade. The main components necessary for this to be accomplished are a vortex generation unit, a test section with a test rotor, and a particle image velocimetry system which are able to synchronize with each other. This synchronization is intended to allow the user to pick a point in time and vertical location on the test rotor at which he or she wishes to observe the BVI event. This powerful tool will allow for any number of noise and vibration reduction rotor modifications to be studied and verified both time and cost effectively.

A personal goal of this work was to master the PIV measurement technique such that this skill could be used in any fluid dynamics application. This required a fundamental understanding of the theory pertaining to the numerous physical components of a PIV system, the mathematical background to comprehend the processing of PIV images and post processing of PIV measurements in addition to the theory behind the flow phenomenon which was to be investigated. 


\section{Chapter 2}

\section{Particle Image Velocimetry}

Particle image velocimetry (PIV) is an indirect, whole fluid flow measurement technique which allows for all velocities within a finite two-dimensional analysis area to be realized simultaneously, without disturbance of the flow. The non-intrusive nature of the measurement is attained by the use of imaging of tracer particle scattered laser light and is an indirect measurement due to the use of a direct measurement of flow tracers to estimate the fluid flow. Two images of the scattered laser light, separated by a brief instant in time, are analyzed in a comparison process which determines the distance that the fluid inside predetermined cells within the measurement area have moved. This information, coupled with the time between images, results in a two-dimensional array of the cell velocity vectors.

The tracer particles, which scatter the coherent laser light, are chosen carefully, as parameters such as density, index of refraction, diameter and concentration are important for accuracy of the measurement. Laser light is used due to the powerful intensities which can be created and the control that the user can have over the beam. Also, the monochromatic laser light can easily be bundled into thin sheets which does not create chromatic abberations when imaged. Laser light is very desirable for particle image velocimetry measurements, but in theory any powerful light source could be used. A sketch of a sample PIV set up within a wind tunnel is shown in Figure 2.1.

As mentioned earlier, the captured images of the illuminated tracer particles must undergo a mathematical comparison to arrive at the velocity information desired. Once this is done, however, these raw vector maps should be post-processed in order to eliminate 


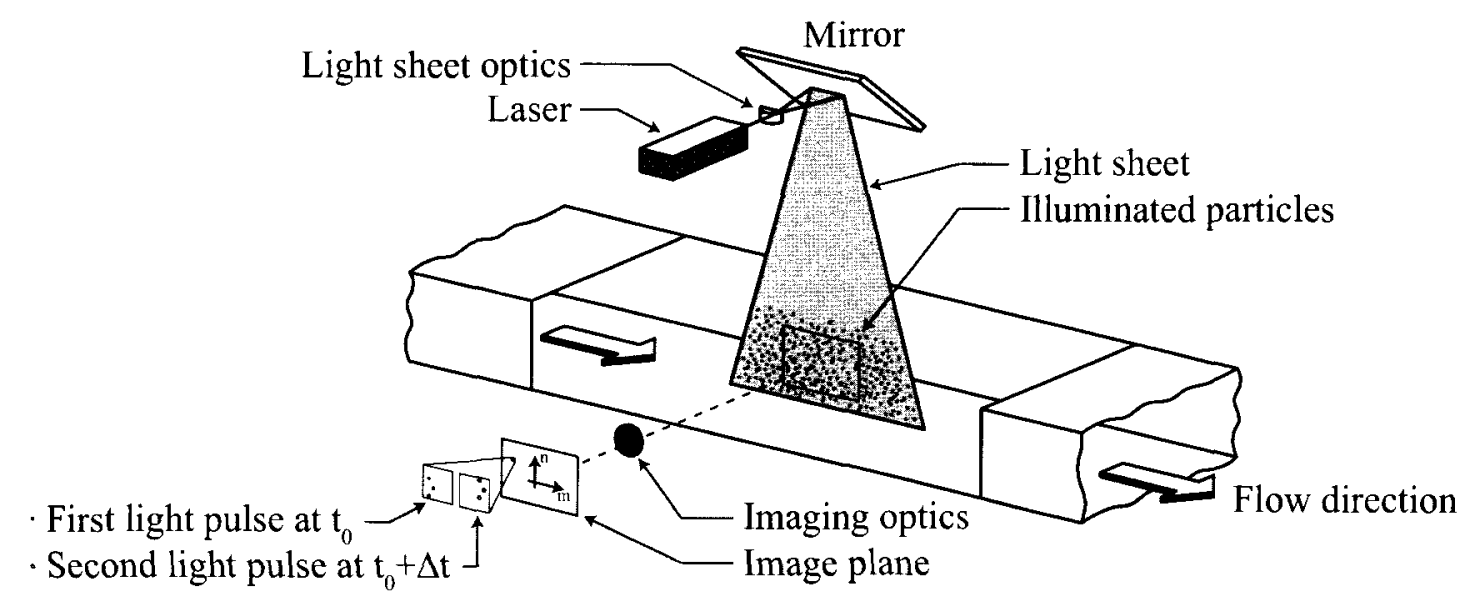

Figure 2.1: Sketch of a sample experimental arrangement for particle image velocimetry measurements within a wind tunnel [2].

artifacts of the measurement which do not reflect the physical flow situation; some of the individual vectors in the vector plot must be discarded and possibly replaced.

After the acquired data has been processed and post-processed, in order to ensure its validity, there are many ways the data can be manipulated and expressed, from velocity to pressure to turbulence, making the particle image velocimetry a very powerful measurement technique in the field of fluid dynamics.

In addition to the comprehensive guide and review of the PIV measurement technique mentioned earlier, various other summaries of different aspects of the technique have been completed. The digital nature of digital PIV has been explored in terms of linear system theory by Westerweel (1997) [8] and reviewed by Willert and Gharib (1991) [9]. The methods used to analyze the image pairs of PIV have been reviewed from a theoretical view point (Keane and Adrian, 1992 [10]) and accuracy of these methods compared (Pust, 2000 [11]).

\subsection{Tracer Particles}

The velocity fields observed through PIV measurements are, in fact, the velocities of the tracer particles which are seeded within the flow. The particles' size and relative density to the fluid are two important characteristics when considering the ability of a tracer particle to accurately follow the streamlines of the flow. A comprehensive review of tracer particles 
in PIV measurements has been completed by Melling (1997) [12], covering particle accuracy in turbulent and transonic flows.

Smaller particles tend to trace the flow more closely, therefore, the smaller the tracer particles, the more accurate the PIV measurements can be. However, these particles tend to not scatter the light as efficiently as larger particles, so the imaging of small particle becomes an issue. This reduces the ability of the PIV system to identify movement within the flow, however accurate that movement is compared to the flow. Increasing the intensity of laser light which is illuminating the flow tracers can compensate for this shortcoming, however, this can become quite expensive given the relationship of the laser's cost with its output power at the levels demanded by particle image velocimetry. A compromise must be made between tracer particle size, what is an acceptable amount of error in the data and the related cost of a more powerful laser illumination system. In general, for most subsonic flows, seeding particles less than $10 \mu \mathrm{m}$ in diameter for gas flows and $50 \mu \mathrm{m}$ in diameter for liquid flows are acceptable tracers, with some allowance depending on the density of the flow medium.

Some commonly used tracer particles for gaseous flows are shown in Table 2.1.

Table 2.1: Common PIV tracer particles used in gaseous flows

\begin{tabular}{lll}
\hline Type & Material & Mean diameter $(\mu \mathrm{m})$ \\
\hline \hline Solid & Polystyrene & $0.5-10$ \\
& Aluminum & $2-7$ \\
& Magnesium & $2-5$ \\
& Glass micro-balloons & $30-100$ \\
& Granules for synthetic coating & $10-50$ \\
& Dioctylphathalate & $1-10$ \\
Smoke & & $i 1$ \\
Liquid & Different oils & $0.5-10$ \\
\hline
\end{tabular}




\subsubsection{Traceability of Suspended Particles}

Since the ability of a PIV measurement to accurately describe the physics of a flow is heavily dependent on the ability of the tracer particle to follow the fluid, the choice of the appropriate tracer must involve consideration of the fluid mechanical properties of two phase flows. Although the interaction between particles and the coupling of the flow to the particles are minimal, the influence of the flow on the particles is large, and ideally perfect. The degree to which the particle motion is coupled to the fluid motion should be maximized and a technique in which to characterize this coupling is necessary.

The one-way coupling between the particle and the fluid can be estimated for spherical particles in a viscous fluid at very low Reynolds number (given by $R e=\frac{V_{\infty} d_{p}}{\nu_{f}}<1$ ) and constant acceleration [2], only considering Stoke's drag, by:

$$
\bar{V}_{p}(t)=\bar{V}_{\infty}\left[1-\exp \left(-\frac{t}{\tau_{s}}\right)\right]
$$

where the relaxation time, $\tau_{s}$, is given by

$$
\tau_{s}=d_{p}^{2} \frac{\rho_{p}}{18 \mu_{f}}
$$

Although for many flow conditions the restriction on $R e$ does not hold, due to larger flow velocities, the settling time $\left(\tau_{s}\right)$ still provides a convenient and accurate indication of the particle's response to a change in flow velocity and its tendency to attain equilibrium with the flow. Using Equation 2.1 with a step acceleration in the flow velocity, Figure 2.2 shows the time response for droplets of oil with various diameters.

In most cases, the flow velocities are large enough to neglect gravitational effects and generally electrostatic forces can be ignored. A more thorough analysis of the unsteady motion of a spherical particle, as derived by Basset in 1888 then expanded on by Hinze in 1959 , in a moving fluid is as follows:

$$
\frac{\pi}{6} d_{p}^{3} \rho_{p} \bar{V}_{p}=-3 \pi \mu d_{p} \bar{V}_{r e l}+\frac{\pi}{6} d_{p}^{3} \rho_{f} \bar{V}_{f}-\frac{\pi}{12} d_{p}^{3} \rho_{f} \bar{V}_{r e l}-\frac{3}{2} d_{p}^{2} \sqrt{\pi \mu \rho_{f}} \int_{t_{0}}^{t} \frac{d \bar{V}_{r e l}}{d \xi} \frac{d \xi}{\sqrt{t-\xi}}
$$




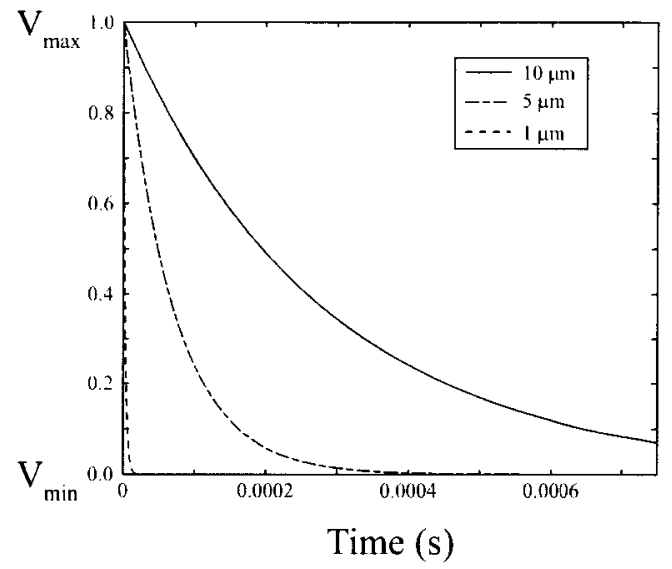

Figure 2.2: Time response of oil particles in air with various diameters in a downward step flow [2].

where $\bar{V}_{r e l} \equiv \bar{V}_{p}-\bar{V}_{f}$ as shown in Figure 2.3. The first two terms describe the accelerating force of the fluid and Stoke's law respectively. The force due to the pressure gradient which is present in an accelerating flow is given by the third term in the equation. The fourth term, following from potential theory, describes the resistance of an inviscid fluid to accelerated motion of the tracer particle. The last term in the equation, the Basset history integral, characterizes the amount of resistance which is observed in the presence of an unsteady flow. By combining the first, third and fourth terms, the result describes an accelerating force on a sphere which has a virtual mass of one half of the displaced fluid in addition to the mass of the tracer particle itself.

In practice, the Stoke's approximation for the motion of tracer particles in the flow provides a conservative estimate and generally under-predicts the influence of the fluid on the spheres. Thus, this handling of the physics provides a conservative criteria for evaluating the appropriateness of a particular choice for seeding of flows for PIV measurements.

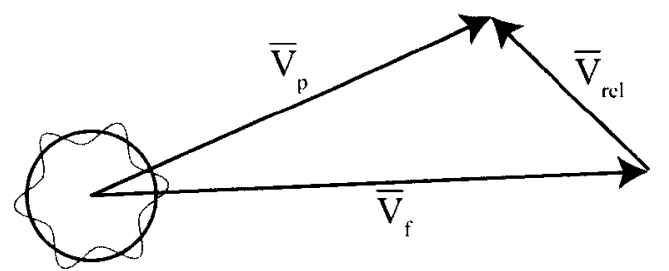

Figure 2.3: Relative velocities of a suspended tracer particle [12]. 


\subsubsection{Light Scattering Characteristics of Small Particles}

In general, the quality of images captured for analysis in PIV (intensity and contrast) are heavily dependent on the amount of light scattered by the seeding particles in the flow as well as the direction at which the scattering occurs. In order to optimize the amount of light that is observed, an understanding of optical scattering by small particles is necessary.

For spherical particle with diameters larger than the wavelength of the incoming light, Mie's scattering theory can be applied. Figure 2.4 shows two cases of Mie scattering of $532 \mathrm{~nm}$ light by (a) $1 \mu \mathrm{m}$ and (b) $10 \mu \mathrm{m}$ particles of oil, having an index of refraction of $n=1.468$, in air. The intensity of the scattered light $(I)$ is plotted with respect to the scattering angle $(\theta)$. It can be seen that the perceived intensity not only varies with the particle size but with the angle at which the light is viewed. The intensity for both particle diameters shown are much greater for near-forward scattering angles when compared to perpendicular scatter $\left(90^{\circ}, 270^{\circ}\right)$. Capturing the light at near $180^{\circ}$ scatter angles would give high intensity levels; however, due to the limited depth-of-field in two-dimensional PIV, images are generally captured at near perpendicular angles to the light sheet.

A useful parameter for characterizing the Mie scattering is the normalized diameter, given by Equation 2.4:

$$
q=\frac{\pi d_{p}}{\lambda}
$$

where $d_{p}$ is the diameter of the tracer particle and $\lambda$ is the wavelength of the incident laser light. In general, for $q i 1$, the intensity profile of the scattered light will have approximately $q$ peaks in the range $0^{\circ}-180^{\circ}$ and for increasing $q$ there will be a larger proportion of forward scattered light. Thus, there is a strong tendency towards higher levels of scattered light for larger tracer particles in the flow.

\subsubsection{Tracer Particle Generation and Delivery}

Depending on the material used to seed a flow for PIV investigation, the method used to generate the particles will vary. For solid particle seeding the material, in the form of a dry powder, can be dispensed using fluidized beds or air jets. Liquid particles can be 


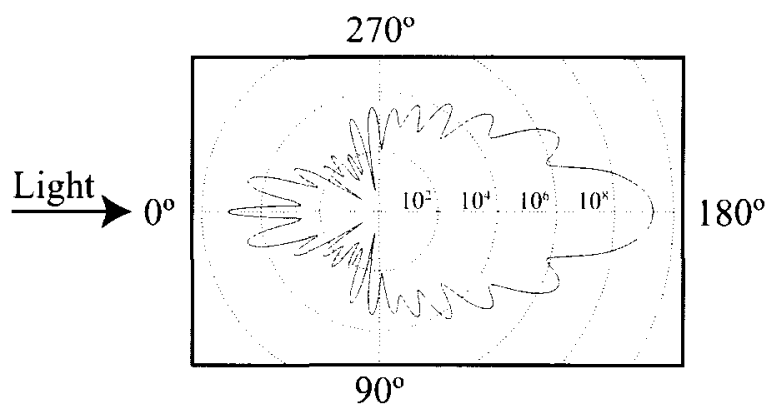

(a)

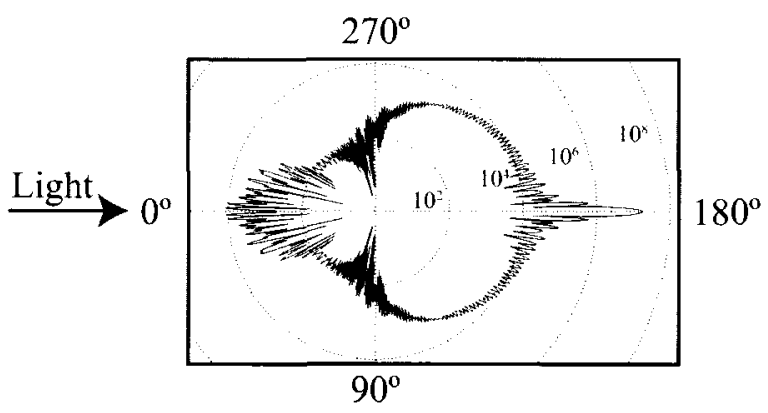

(b)

Figure 2.4: Mie scattering of $532 \mathrm{~nm}$ incident light for olive oil droplets with diameter (a) $1 \mu \mathrm{m}$ and (b) $10 \mu \mathrm{m}[2]$.

created using an evaporator-condensation approach or an atomizer. In the literature, most studies within wind tunnels have been done using Laskin nozzle atomizers with oil as the material, which generally give a polydisperse aerosol with a relatively small mean diameter. A schematic of a standard Laskin nozzle atomizer can be seen in Figure 2.5.

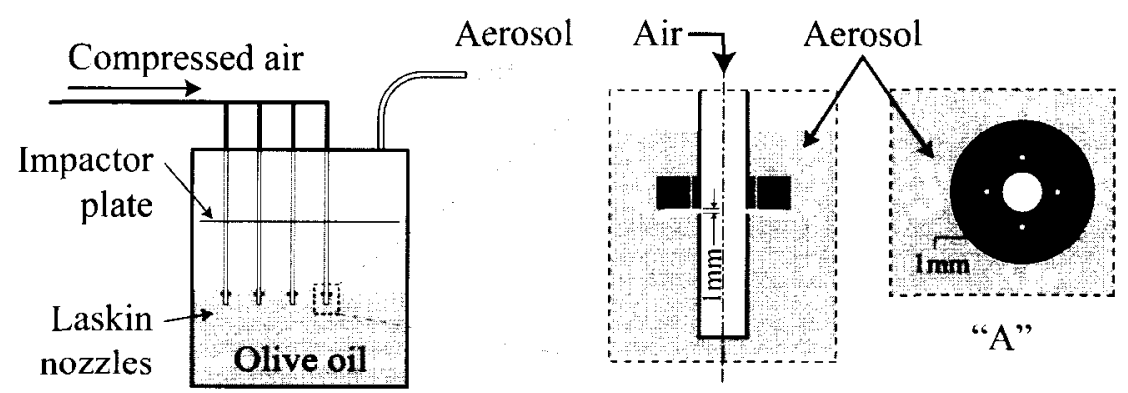

Figure 2.5: A schematic of an aerosol generator with Laskin nozzles for PIV tracer particle creation.

The aerosol generator is comprised of a set of air supplies which enter a container filled 
with the particle medium, in this case an oil such as olive oil, a set of Laskin nozzles in a pipe which is connected to the set of air supplies, a secondary air inlet which is not connected to a Laskin nozzle (optional) and an outlet for the aerosol to exit the chamber. The inlet pipes (four in the schematic in Figure 2.5) are submerged into the aerosol medium and capped at the submerged end. A set of equally spaced small $(\approx 1 \mathrm{~mm})$ holes, the Laskin nozzles, are located around the pipe under the liquid surface. As the jet of air is forced into the liquid, bubbles form and rise to the surface. Within these air bubbles, droplets of the liquid aerosol medium will form due to the shear stress created by the sonic jets from the Laskin nozzles. As the bubbles reach the surface and pop, the aerosol is released into the chamber. Generally, an impaction plate is located between the liquid surface and the outlet leaving a small $(\approx 2 \mathrm{~mm})$ gap between it and the container walls such that larger particles in the aerosol will deposit and return to the reservoir, leaving only the smaller particles in the aerosol. This process can also be achieved after particles leave the generator, using separate a particle impactor, if the particles are not of sufficiently small mean diameter.

The choice of material for the generation of tracer particles, along with pressure differential of the inlet supply, will affect the size of the resulting aerosol particles. In PIV, olive oil is the most common choice due to the acceptably small particles created (mean diameter of about $1 \mu \mathrm{m}$ ) and the lower health risk to the experimenter if the study is being conducted in an open test section, when compared to other possible tracer particle mediums.

The tracer particles must somehow be introduced into the flow with as little disturbance as possible. In much of the literature this is done using a rake, or a pipe with many holes located along its length, placed into the flow and through which the tracer particle aerosol is passed. The particles must be introduced far enough upstream to achieve sufficient dispersion and uniform concentration throughout the flow by the time they reach the measurement area. In closed-circuit wind tunnels, it is possible for the tracers to be introduced downstream of the test section and allowed to recirculated back to the measurement point similar to the method used by Smith et. al (1992) [13]. This has the advantage of little to no disturbance of the flow and good allowance for completely uniform distribution of the tracers in the flow at test section. Another advantage is that larger, undesirable 
particles will be more likely to leave the flow, effectively lowering the mean diameter of the tracer particles that reach the test section. However, given this point, it must be ensured that there is a large enough initial concentration such that the seeding at the test section is sufficient for PIV measurements.

\subsection{Laser Theory}

Since laser sources are the most widely used illumination method in particle image velocimetry and is the type used in the current study, only these light sources will discussed here. More specifically, the laser of choice for modern PIV systems is the Nd:YAG laser, and will be the topic of further discussion on lasers in particle image velocimetry.

A laser, or Light Amplification by Stimulated Emission of Radiation, is composed of three main components: The lasing material, a pump energy source and a pair of oppositefacing mirrors. A schematic of a simple laser is shown in Figure 2.6. The laser material is the element that is responsible for the emission of light and its properties determine the characteristics of the output. For the Nd:YAG laser this is a rod of Neodymium doped yttrium-aluminum-garnet crystal, where the Neodymium ions $\left(N d^{3+}\right)$ are the component responsible for the laser light emission and the yttrium-aluminum-garnet crystal defines the allowed energy levels in the system. The pump source excites the laser material by introduction of some form of energy, usually electromagnetic, into the material. It is this energy with which the laser material is able to emit light. A pair of inward-facing mirrors are placed at either end of the lasing medium so that oscillation of light back and forth through the medium can occur, resulting in the amplification of light. One of the mirrors will allow partial transmission such that the light can escape to become the laser beam.

From quantum mechanics it is know that three types of atomic interactions with light are possible. Figure 2.7 shows diagrams of these three interactions using an simple energy level diagram of a hypothetical atom in a laser material having only two discrete energy levels. The first type of interaction, absorption, occurs when an incoming photon, of the appropriate energy, hits an atom in its ground state $\left(E_{1}\right)$, the photon is absorbed and 


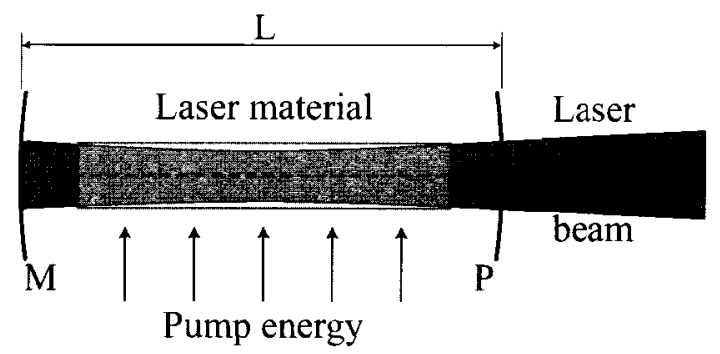

Figure 2.6: A schematic of a simple laser cavity showing the laser material, the pump energy source and the mirrors [2].

the atom is pushed into an excited state $\left(E_{2}\right)$. Once an atom is in an excited state, it will most likely fall back to the ground state. This decay, the second type of interaction called spontaneous emission, results in a photon being emitted from the decaying atom in a random orientation with the energy given by Equation 2.5:

$$
E_{\text {photon }}=E_{2}-E_{1}=h \nu
$$

where $\nu$ is the photon's frequency and $h$ here is Plank's constant. However, in the third type of interaction, if the incoming photon has the frequency $\nu$ and the atom is in the excited state, $E_{2}$, then it is possible that the incoming photon could stimulate the atom into a non-spontaneous transition back to the ground state, $E_{1}$, in a process called stimulated emission. The emission of a second photon of equal frequency, and thus energy, will be perfectly in phase with the initial photon. This, in terms of waves, coherently amplifies the incoming wave. It is this type of interaction which is responsible for the emission of light in lasers.

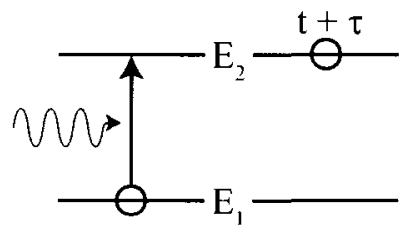

Absorption $\mathrm{E}_{2}-\mathrm{E}_{1}=\mathrm{hv}$

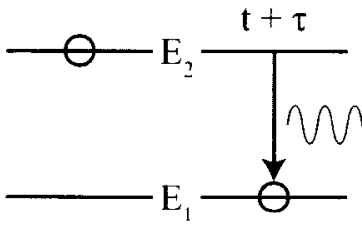

Spontaneous emission hv

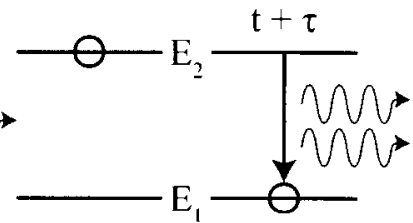

Stimulated emission $2 \mathrm{hv}$

Figure 2.7: A diagram of a hypothetical atom in a two-state energy system. Three possible atom-photon interactions are shown: absorption, spontaneous emission and stimulated emission [2]. 
Laser-type light output will only occur if there is a majority of the atoms in the laser material which are suddenly elevated to the $E_{2}$ state, or, in other words, when $N_{2} \& N_{1}$ in the laser material. This state is not stable, and will result in the massive cascade of atoms back into the $E_{1}$, or ground, energy state and thus the emission of large amounts of light. Thus, to force a population inversion $\left(N_{2} \& N_{1}\right)$ in a laser medium at steady state $\left(N_{1} \approx N_{2}\right)$, the laser medium must be pumped with an external energy source. The Nd:YAG laser generally is pumped with a burst of electromagnetic radiation from a flashlamp in order to raise the $N d^{3+}$ ions in the medium to an upper energy state, but other laser mediums use electrical or chemical stimulation. Since the photons from this burst will go through a cascade of absorption and random direction spontaneous emissions as they make their way through the medium, an elevated energy state status will also radiate through the medium. Ideally, this process will raise a majority of the $N d^{3+}$ ions to a state of greater energy than the upper energy lasing state of the $N d^{3+}$, which has more levels than the two-state system shown in Figure 2.7.

A laser medium with only two energy levels would not work, since when $N_{1} \approx N_{2}$ the absorption of a $E_{2}-E_{1}$ photon is just as likely to occur as the emission of a photon of the same energy. At least three separate energy states are required for a true and useful population inversion to occur. In this manner, photons with a larger amount of energy than the upper-lasing state (middle energy level in a three state system) will be absorbed, putting the atom into the highest state. This highest state then decays to the upper-lasing state without photon emission. This state, through spontaneous or stimulated (ideally) emission, then emits a photon which will not be reabsorbed. Even in a three-state laser, however, lasing requires excessive pumping to excite enough atoms into elevated states such that the ground state is absent enough that the atoms will rush back. A four-state or greater system is even better, as the pumped atoms can fall down to a lower-lasing energy state that is separate from the ground state and thus much more sparsely filled.

Nd:YAG lasers have multiple energy states, of which there are four levels of interests for PIV lasers light creation. A simplified energy state diagram for $N d^{3+}$ is shown in Figure 2.8. As the Nd:YAG rod is pumped, most the atoms in the rod are elevated to the pumped state 
$\left(E_{4}\right)$. There is then a quick and non light emitting transition to the metastable upper lasing state $\left(E_{3}\right)$ where the atom remains for a relatively long time. As the atom emits photons with the wavelength of $1064 \mathrm{~nm}$ which can then be frequency doubled to give radiation of the wavelength of $532 \mathrm{~nm}$. This is in the green band of the electromagnetic spectrum and more useful for PIV measurements with visible range CCD cameras.

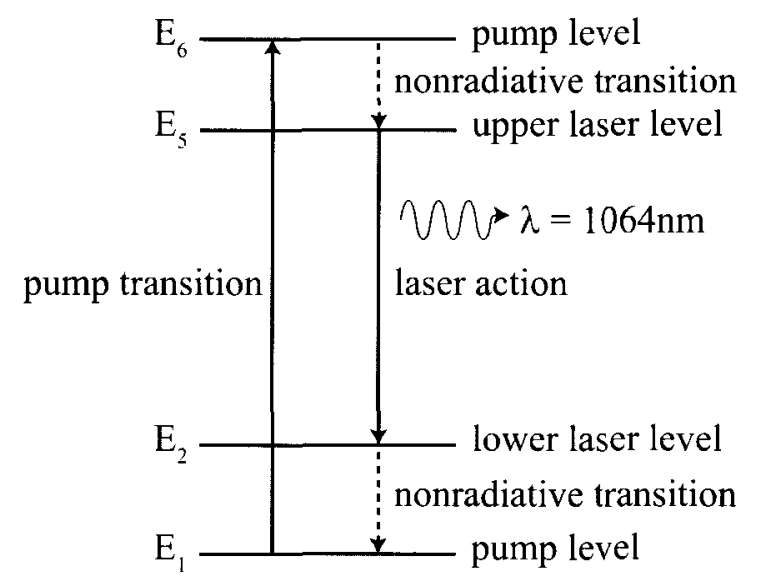

Figure 2.8: The simplified energy level diagram for $\mathrm{Nd}^{3+}$. Only energy levels which are important for the emission of $1064 \mathrm{~nm}$ light are shown [2].

In order to maximize the light output of a laser, the lasing material, which is usually a long cylindrical rod, is enclosed with mirrors at either end to allow for the photons to cycle through the material and amplify many times. This is the arrangement that is shown in Figure 2.6. The output mirror will have a slightly transmissive coating on the inside to allow light to pass and become the laser beam, while the back mirror is located at the other end of the laser cavity and has a very reflective coating. As the cascade of stimulated photons builds up, the photons that are in the longitudinal direction of the rod will reflect back and forth between mirrors and be amplified via stimulated emissions during each pass successive through the medium. This avalanche of photons, all perfectly in synchronization with each other and of equal wavelength, will shortly reach a stationary process which can be released all at once as a pulse or over time as a continuous wave. Pulsed lasers tend to be much more powerful due to the ability to release all of the energy present within the laser at once, compared to the continuous emission of a steady state laser beam. Since PIV 
measurements benefit from very powerful illumination of tracer particles, a very powerful pulse of light is preferred. This presents another problem since two pulses are required and the repetition rate for the lasers is often not fast enough to satisfy the short time duration between PIV images. In order to overcome this, many PIV systems will operate with two $\mathrm{Nd}$ :YAG lasers aligned to illuminate the same plane in the flow, temporally separated by as little time as necessary.

With the PIV Nd:YAG lasers, a quality switch (Q-switch) is often implemented in order to restrict the emission of light until a narrow band of time where the flashlamp cycle is most energetic. This results in a very intense and short pulse of laser light. The Q-switch generally consists of a polarizer plate, a beam-path correcting prism and a Pockels cell. The Pockels cell is a birefringent medium which can be voltage-controlled. The Q-switch is located between the back, highly reflective mirror and the laser rod. A simplified schematic of the implementation of the Q-switch is shown in Figure 2.9. At the beginning of the pumping action by the flashlamp, the voltage on the Pockels cell is turned off. This allows it to exhibit its birefringent property as a quarterwave plate, which rotates the polarization of the light passing through it by $90^{\circ}$. The polarizer then rejects this beam of light since its polarization is now $90^{\circ}$ rotated, and the Q-switch is closed. When the amount of photonic energy stored in the laser rod peaks at the most intense point of the flashlamp pumping cycle, the Pockels cell voltage is turned on, removing its quarterwave plate property. The light passes through it and through the polarizer unaffected and the Q-switch is opened. Photon oscillation within the laser resonant cavity commences and the massive amount of energy stored in the laser rod is released in a very short pulse (on the scale of nanoseconds) after which the voltage to the Pockels cell is turned off, closing the Q-switch and stopping all light emission by the laser.

Since most commercially available imaging devices are most sensitive in the visible band of light, the $1064 \mathrm{~nm}$ light that is emitted by the Nd:YAG lasing crystal must be frequency-doubled. The laser beam is passed through a second harmonic generator halving the photons' $1064 \mathrm{~nm}$ wavelength to $532 \mathrm{~nm}$, effectively converting infra-red light into green visible light. The separation of the frequency doubled light results in a loss of two-thirds of 

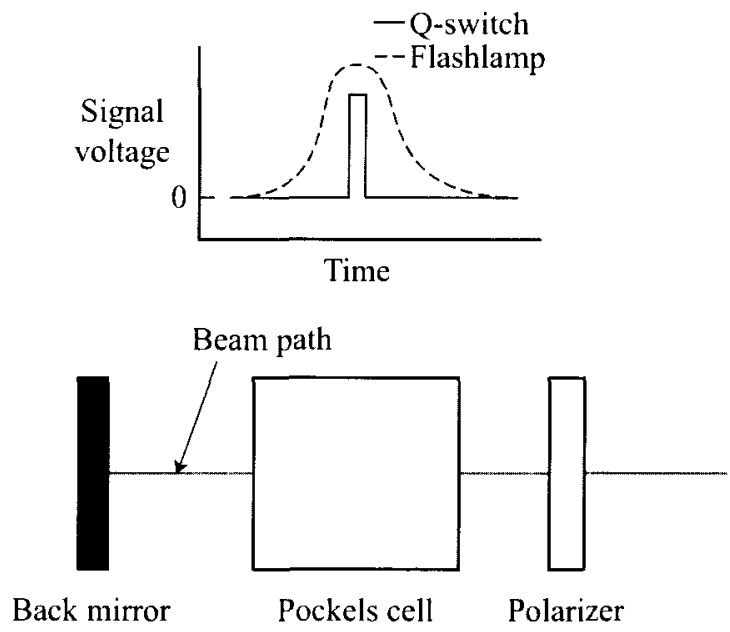

Figure 2.9: A simplified schematic of the Q-switch as implemented in a Nd:YAG laser resonant cavity.

the original laser power provided.

\subsection{Charge-Coupled Device Camera Theory}

In digital particle image velocimetry (DPIV), the images are captured using a digital chargecoupled device (CCD) camera and converted to a bitmap for processing. The speed at which measurements can be made with digital photography have made it an ideal choice for PIV. Since the accuracy of the images that are used to create the vector maps is of great importance for the accuracy of the overall measurement, a clear understanding of the theory behind digital photography is necessary.

The CCD sensor is a solid state element that is about $10 \times 10 \mu \mathrm{m}^{2}$ in size. A crosssection schematic of an ordinary CCD pixel is shown in Figure 2.10. The CCD chip, or array of many CCD elements, is generally built upon silicon due to its semiconducting properties. The pixel is comprised of metal contacts on the surface, below which is an insulating oxide layer. Beneath this is a layer of semiconducting material that has been n-doped, and that is on top of a layer which has been p-doped. A small voltage is applied across the metal contacts and an electric field is generated.

When an incident photon falls on the CCD sensor it is absorbed by a bound electron, 


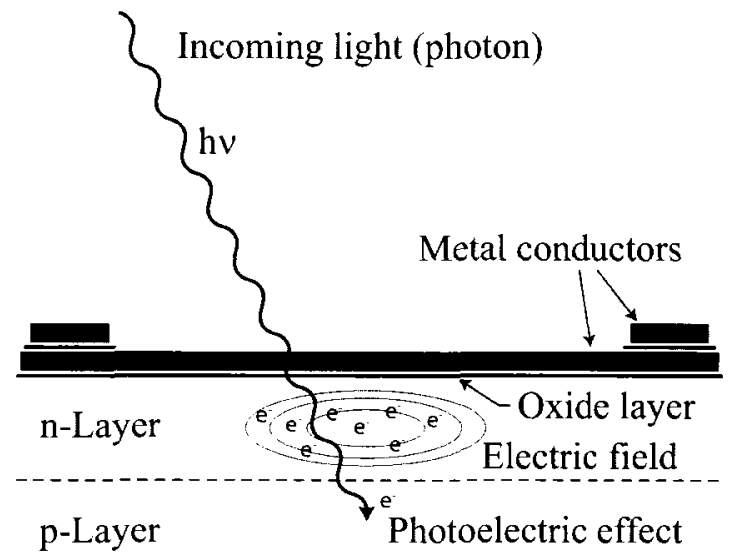

Figure 2.10: A simplified schematic of a pixel in a CCD camera [2].

freeing it from its orbit and generating an electron-hole pair; a process known as the photoelectric effect. The hole is absorbed in the p-doped layer while the electron migrates to the potential well created by the electric field, where it remains. The more photons that arrive at the p-n junction, the more electrons that build up in the potential well, and the more charge there is. Since the amount of charge varies linearly with the voltage, the voltage can be read out and converted to number of photons or intensity of light that was captured in that specific CCD pixel.

The time required to empty the pixels of the charge that has accumulated is a problem for PIV measurements. This read time is generally longer than the time between successive images of a single measurement, such that the CCD camera is not ready to take the second image $\Delta t$ seconds after the first. To overcome this, newer CCD cameras intended for PIV use an architecture called full-frame interline transfer for the CCD elements. These CCD elements each have a storage area attached to them so that the data acquired during the first pulse can be quickly transferred to the storage and the CCD can then acquire a second set of data. Since the time required to transfer the first image's data to the local storage is less than $1 \mu \mathrm{s}$, this method allows for a very short $\Delta t$ and is satisfactory for PIV use. A schematic of the progressive scan interline transfer CCD layout can be seen in Figure 2.11.

Some sources of noise or inaccuracy in the image that the array of CCDs produce include overflow of photons (or electrons) into neighbouring pixels, thermal effects and 


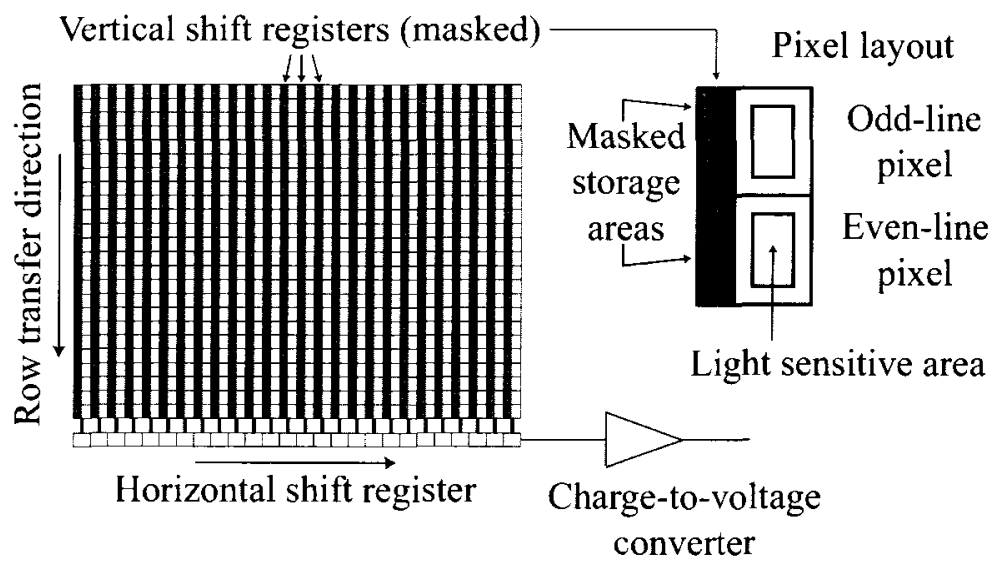

Figure 2.11: A schematic of a pixel array in a full-frame interline transfer CCD camera [2].

charge-to-voltage errors. Heat will also cause the generation of electron-hole pairs in the CCD which will be interpreted at photon activity. The rate at which electron-hole pairs generate doubles for every $6-7^{\circ} \mathrm{C}$ increase in temperature. Peltier cooled cameras, cooling the CCD chip electronically, are commonly used to avoid this problem.

\subsection{Particle Image Velocimetry Image Processing}

Once a pair of images have been captured, they must be processed to attain the velocity vector maps which estimate the flow characteristics in the measurement area. The images are divided into a grid of cells, or interrogation regions, which are relatively small when compared to the size of the entire image. The number of vectors in the final grid of velocities will be the same as the grid of interrogation regions, with one velocity vector for each interrogation region. Each pair of interrogation regions (one from each of the two images) are individually analysed in a correlation process to obtain an average displacement of seeding particles. This represents the distance that the particles have traveled in that interrogation region over the time that temporally separate the images $(\Delta t)$. This displacement is then used to calculate an estimated average speed and direction which that cell had during the measurement.

There are two common methods by which PIV images are processed. Cross-correlation is used when two separate exposures are captured of the flow at two different temporal 
locations. Auto-correlation is used when the two images cannot be taken separately but rather the film is doubly exposed at the two temporal locations resulting in one image with both the particles' start and end positions present.

Auto-correlation was used extensively in the past when the equipment required to grab two sequential frames microseconds apart did not exist. Now, CCD cameras are available that have overcome this hurdle and cross-correlation is widely accepted as the preferred image processing technique for PIV. Furthermore, in order to reduce computational time required for digital particle image velocimetry (DPIV) image analysis, a fast-fourier transform (FFT) technique is commonly used to compute the cross-correlation. This method exploits the signal-like nature of a digital image by performing them in the spatial frequency domain, reducing the number of calculations necessary.

A simplified illustrated flow chart is shown in Figure 2.12 which describes the process by which a PIV measurement is made and processed. The three main operations necessary for a PIV measurements are displayed. The two images above the label $A$, are captured $\Delta t$ seconds apart in time, of the same, briefly illuminated, region of the flow field. These two images are then broken up into smaller regions, the interrogation regions, at point $B$. These regions are of size $N_{x}$ wide by $N_{y}$ tall. The regions do not have to be square, not do they have to remain the same size through out the image (they generally are for simplicity), but they must be the same in each of the two images. Each interrogation region-pair (one from each image in the same location) is then compared with each other at point $C$, through a correlation process. This correlation process determines the average displacement of the fluid element within that interrogation region by observing the particles' shifts over the $\Delta t$. This will give the velocity of that fluid element, and thus a single vector. This procedure is repeated for every interrogation region-pair to arrive at an array of velocity vectors for the full image area of the flow.

\subsubsection{Cross-Correlation}

The statistical technique of spatial cross-correlation is the method most commonly used for current PIV image analysis in order to approximate the fluid flow behaviour from an image 


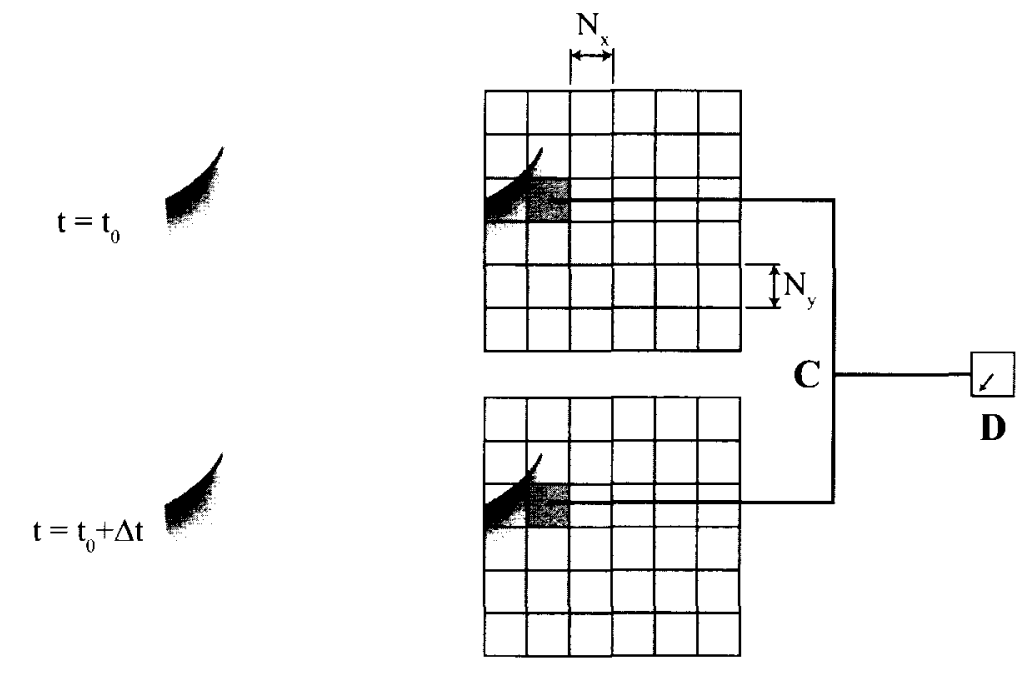

A

B

Figure 2.12: A simplified illustrated flow chart showing the three basic, essential, steps to the PIV method: Image-pair capture, interrogation region selection, correlation.

pair of illuminated tracer particles entrained in the flow. The cross-correlation technique is generally accepted to be the superior [10] technique for PIV when compared to autocorrelation. In order to estimate the flow velocities using cross-correlation, two separate images-one of the start locations of the particles and one of the final locations-are required. The difference between the two images is a certain amount of spatial shift during a brief amount of time $(\Delta t)$ that elapsed between the consecutive image captures. A linear signal processing block diagram which describes this spatial shift of the particles between images is shown in Figure 2.13.

The functions $f(m, n)$ and $g(m, n)$ represent the discrete intensity profiles of interrogation regions within the first and second images, respectively. The Additive noise process, $d(m, n)$ represents the noise generated when particles enter or leave the interrogation region during the time, $\Delta t$, between the consecutive images described by $f$ and $g$. The second intensity profile, $g$, can be thought of as the output of the spatial shift function, $s(m, n)$, applied to the input intensity profile, $f$, plus the additive noise. The coordinates $(m, n)$ are discrete locations in the image plane, in units of pixels. The capitalized functions represent their lower case counterpart but in the spatial frequency domain via a fast fourier transform. 


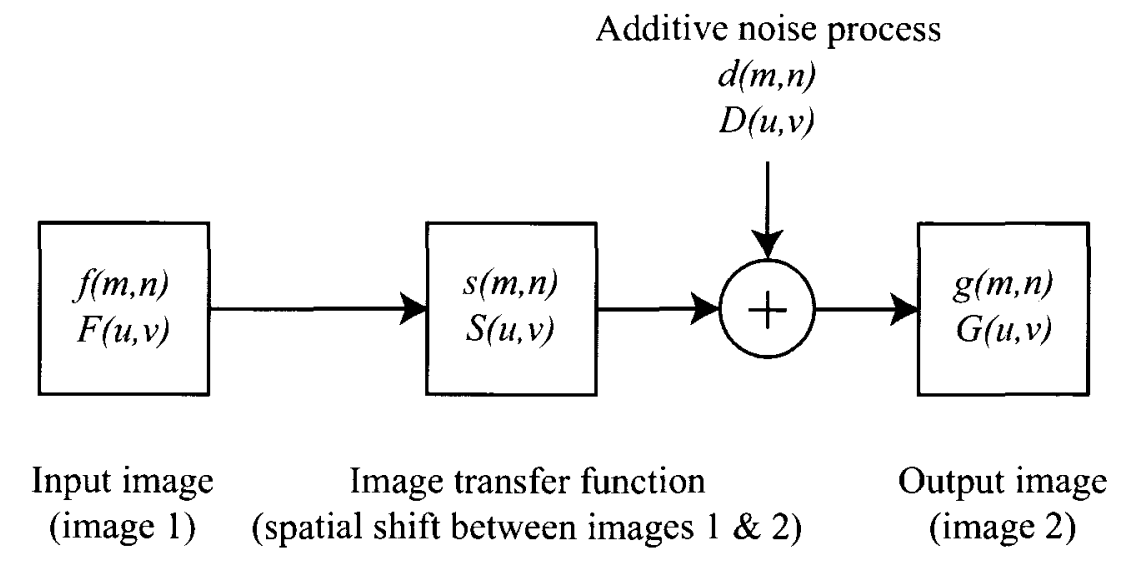

Figure 2.13: Spatial cross-correlation block diagram [9].

For example:

$$
F(u, v)=\mathcal{F}[f(m, n)]
$$

where the coordinates $(u, v)$ describe locations in the spatial frequency domain.

The goal in PIV is to estimate the spatial shift function, $s(m, n)$, by using the statistical method of spatial cross-correlation to obtain an average displacement of interrogation regions which represent the region captured in the measurement area. The discretized crosscorrelation function $\phi_{f g}(m, n)$ (discretized due to the wide use of CCD cameras for imaging) of the interrogation regions $f$ and $g$ is given by Equation 2.7:

$$
\begin{aligned}
\phi_{f g}(m, n) & =\mathcal{E}[f(m, n), g(m, n)] \\
& =\sum_{k=-\infty}^{k=\infty} \sum_{l=-\infty}^{l=\infty} f(k, l) \cdot g(k+m, l+n)
\end{aligned}
$$

where $l$ and $m$ are dummy variables used to compute the correlation function.

The result of the cross-correlation of $f$ and $g$, is $\phi_{f g}$, or the cross-correlation plane. This plane is a two-dimensional surface with the height at $(m, n)$ given by its correlation coefficient, $\phi_{f g}(m, n)$. This surface will likely be noisy with a sharp, relatively large peak at some point. This peak represents the location, in the correlation plane, where the two interrogation region intensity profiles correlated the best. More generally, this peak is 
located at a position $\left(m_{0}, n_{0}\right)$ in the cross-correlation plane where that position represents the amount of shift (in pixels) in both directions that was necessary to overlap the particles in the two interrogation region images with the best fit; this position represents the average particle displacement for that interrogation region.

Obviously, the likelihood is low that the two intensity profiles will overlap perfectly at any point in $(m, n)$ space since the particles move independently of each other during the $\Delta t$ between images. The unavoidable additive noise which is present due to particle entering or exiting the interrogation regions between imaging compounds the improbability of perfect correlation. When there is high correlation between a particle in $f$ and its spatially shifted partner in $g$, this is called true correlation. When particles in $f$ match up with different particles in $g$, this results in a lower correlation and is called random correlation. However, the best match, or largest peak in the cross-correlation plane, will give a good approximation of the spatial shift function when many particles find their counterparts at the same peak in $(m, n)$ space. From this, and the knowledge of $\Delta t$, the "velocity" of the interrogation region is defined by Equation 2.8 using the image scale factor, $S$ :

$$
V(\vec{x}, y)=S\left[\frac{V_{x} \hat{x}+V_{y} \hat{y}}{\triangle t}\right]
$$

\subsubsection{Auto-Correlation}

Before CCD cameras capable of capturing and storing two sets of pixel arrays within a very short time (on the order of a few microseconds) were readily available, PIV image analysis was performed using auto-correlation. The tracer particles in the flow were illuminated in the same manner, two pulses separated by $\Delta t$, but only one (much longer) exposure was taken. The result was both the initial and final positions of the particles being contained in one image. This created ambiguity, since it would be not be possible to tell which was the initial position and which was the final position. The auto-correlation function is identical in form to the cross-correlation function, with the two interrogation region intensity profiles being taken from the same image instead of the separate initial and final frame images (see 
Equation 2.9).

$$
\begin{aligned}
\phi_{f g}(m, n) & =\mathcal{E}[f(m, n), f(m, n)] \\
& =\sum_{k=-\infty}^{k=\infty} \sum_{l=-\infty}^{l=\infty} f(k, l) \cdot f(k+m, l+n)
\end{aligned}
$$

Essentially, auto-correlation is the cross-correlation of an image with itself. The correlation plane resulting from the auto-correlation process will always have the largest peak at $(l, k)=(0,0)$ indicating (incorrectly) zero displacement. This perfect correlation is due to the tracer particles in the initial positions correlating with themselves in that same position and likewise with the final locations. This does not represent physically stationary flow, or any physical nature of the flow at all, but an artifact of the method used. There will be two identical secondary peaks, at complementary points reflected through the origin of the correlation plane which represent the physical motion of the particles. The two peaks are a result of the particles' initial positions correlating with the final positions, and vice versa; the two peaks describe the same motion, but one describes the forward motion and the other reverse motion. Since they will give equal but opposite displacement predictions, the absolute displacement can be found without ambiguity and the direction will have to be interpreted with knowledge of the flow.

This directional ambiguity is one of many reasons why cross-correlation is the preferred method, as the inability to determine direction excludes it from being used in recirculating flows. Another downside to auto-correlation arises due to the presence of the central selfcorrelation peak in the correlation plane. If shifts of less than about two to three pixels are observed, the peaks that arise due to proper correlation will fall too near the larger central peak and will not be detected. This effectively reduced the dynamic velocity range of the auto-correlation method, a problem cross-correlation does not suffer from.

\subsubsection{Peak Detection and Sub-Pixel Resolution Via Interpolation}

Since the intensity profiles of the images are discrete in DPIV (limited by the resolution of the CCD camera available) the input interrogation region functions $f$ and $g$ are also 
discrete. Obviously, this results in a discrete correlation plane with one pixel (or a region of pixels) holding the peak value. If these discrete coordinates are used, the resulting average displacement will also be discrete and the number of possible interrogation region velocities becomes a small set, an effect known as peak locking. This is a highly undesirable effect and so the peaks in the correlation plane are interpolated using a Gaussian curve fit and a parabolic interpolation to supplement. This results in a peak value which has sub-pixel resolution and effectively allows a much larger range of velocities to be discerned. The peak in the correlation plane is interpolated by assuming a Gaussian fit as such:

$$
p(m, n)=h_{\phi} \cdot \exp \left(-4 \frac{\left(m-m_{0}\right)^{2}+\left(n-n_{0}\right)^{2}}{w_{\phi}^{2}}\right)
$$

where $\left(m_{0}, n_{0}\right)$ is the peak-centre, $h_{\phi}$ is the peak height and $w_{\phi}$ is the peak width at the $e^{-1}$ level or $37 \%$ of the peak height. The primary information is the peak-centre which will contain the displacement data. Equation 2.10 can be separated and the partial derivatives found:

$$
\begin{gathered}
p(m, n)=h_{\phi} \cdot \exp \left(-4 \frac{\left(m-m_{0}\right)^{2}}{w_{\phi}^{2}}\right) \cdot \exp \left(-4 \frac{\left(n-n_{0}\right)^{2}}{w_{\phi}^{2}}\right) \\
\frac{\partial}{\partial m} p(m, n)=h_{\phi} \cdot \frac{-8}{w^{2}}\left(m-m_{0}\right) \cdot p(m, n) \\
\frac{\partial}{\partial n} p(m, n)=h_{\phi} \cdot \frac{-8}{w^{2}}\left(n-n_{0}\right) \cdot p(m, n)
\end{gathered}
$$

Thus, $m_{0}$ can be estimated without knowing $n_{0}$, and vice-versa. However, $p(m, n)$ must first be interpolated from the correlation plane values. This is done using a parabolic interpolation. Once a local maximum $\left(m_{m}, n_{m}\right)$ is found, and the eight surrounding values known $\left(\left(m_{m} \pm 1, n_{m} \pm 1\right),\left(m_{m} \pm 1, n_{m}\right),\left(m_{m} \pm 1, n_{m} \pm 1\right)\right)$, the value of $m_{0}$ and $n_{0}$ can be estimated. The Gaussian peak can be approximated by applying $\exp (x) \approx 1+x$ to Equation 2.11 such that:

$$
p(m, n) \approx h_{\phi} \cdot\left(1-4 \frac{\left(m-m_{0}\right)^{2}}{w^{2}}\right) \cdot\left(1-4 \frac{\left(n-n_{0}\right)^{2}}{w^{2}}\right)
$$


Since $m_{0}$ and $n_{0}$ can each be estimated without knowledge of the other, interpolation in the m-direction is as follows and can be likewise applied in the n-direction:

$$
\begin{gathered}
\left.h_{n m} \equiv h \cdot\left(1-4 \frac{\left(n_{m}-n_{0}\right)^{2}}{w_{n}^{2}}\right)\right) \\
\left.A \equiv f\left(m_{m}-1, n_{m}\right)=h_{n m} \cdot\left(1-4 \frac{\left(\left(m_{m}-1\right)-m_{0}\right)^{2}}{w_{m}^{2}}\right)\right) \\
\left.B \equiv f\left(m_{m}, n_{m}\right)=h_{n m} \cdot\left(1-4 \frac{\left(m_{m}-m_{0}\right)^{2}}{w_{m}^{2}}\right)\right) \\
\left.C \equiv f\left(m_{m}+1, n_{m}\right)=h_{n m} \cdot\left(1-4 \frac{\left(\left(m_{m}+1\right) m_{0}\right)^{2}}{w_{m}^{2}}\right)\right)
\end{gathered}
$$

From this, it follows that:

$$
\begin{gathered}
m_{0}-m_{m}=\frac{1}{2} \cdot \frac{C-A}{2 \cdot B-A-C} \\
h_{n y}-B=\frac{1}{4} \cdot(C-A) \cdot\left(x_{0}-x_{m}\right) \\
w_{m}^{2}=\frac{8 \cdot h_{n m}}{2 \cdot B-A-C}
\end{gathered}
$$

\subsubsection{Fast Fourier Transform Correlation}

From signal processing theory, it is known that a convolution in the time (or spatial) domain is equivalent to a multiplication in the frequency (or spatial frequency) domain. Since it is quite computationally expensive to perform many cross-correlation calculations via convolution (see Table 2.2), it is attractive to do the math in the spatial frequency domain and increase speed at which PIV images can be analyzed. The revised cross-correlation function becomes:

$$
\begin{aligned}
\phi_{f g}(m, n) & =\mathcal{F}^{-1}\left[\mathcal{F}(f(m, n)) \cdot \mathcal{F}^{*}(g(m, n))\right] \\
& =\mathcal{F}^{-1}\left[F(u, v) \cdot G^{*}(u, v)\right]
\end{aligned}
$$

The cost for this vast reduction in computational effort is an increase in background 
Table 2.2: Comparison of the fast fourier transform and direct cross-correlation methods with respect to computational expense.

\begin{tabular}{ll}
\hline Method & Operations \\
\hline \hline Direct & $O\left[N^{4}\right]$ \\
FFT & $O\left[N^{2} \ln N\right]$ \\
\hline
\end{tabular}

noise, most significantly at the boundaries of the interrogation regions. This is due to the requirement that the input to the FFT is infinite in both directions. For the FFT to work, the interrogation region is assumed to be periodic in both spatial directions. Since the interrogation regions are certainly not infinite in span, or periodic in nature, the forced periodicity leads to discontinuities at the boundaries of successive appended cells. This causes problems with the FFT, since the mathematical procedure does not behave well with discontinuities. Also, more importantly, particle images in appended interrogation windows will cause phantom correlations, as particles will correlate with particles in the appended cells; a purely non-physical result of the treatment. There are, however, window and filter functions which can be integrated to alleviate these problems and are discussed later in this section. Another limitation inherent to performing these calculations in the spatial frequency domain is due to the Nyquist sampling criterion of discrete fourier transforms. This effectively limits the available particle displacement in the interrogation region to half of the interrogation region side length $\left(N_{i}\right)$, or $N_{i} / 2$, reducing the dynamic velocity range of the measurement. A flow chart of the FFT cross-correlation process can be seen in Figure 2.14 .

Furthermore, a maximum particle displacement of $N_{i} / 4$ is recommended in the literature in order to achieve a reasonable signal-to-noise ratio, especially when the aforementioned window and filter functions are integrated. This recommended limitation can be exceeded by increasing the concentration of tracer particles present in the interrogation region, causing an increase in true correlations and a reduction in overall noise in the measurement. Another issue related to the FFT method is the restriction it places on the size of the interrogation regions used. Interrogation regions must be square and have lengths that are multiples of 2 
px. These restrictions can be overcome by zero-padding the image areas such that they meet these size requirements, but this leads to more artifacts in the FFT due to the discontinuities which appear at the boundary of the actual image and the zero-padded region.

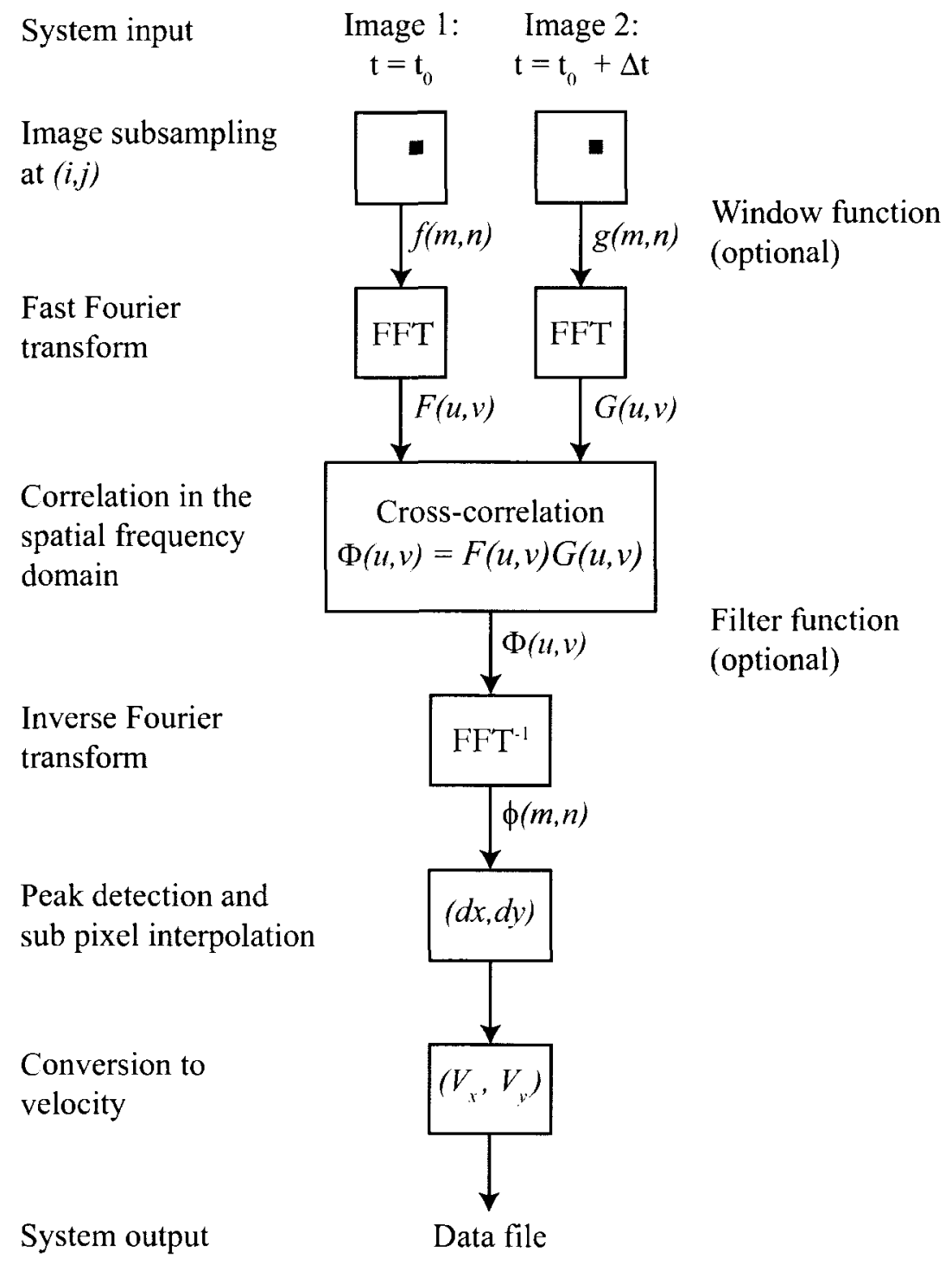

Figure 2.14: Fast fourier transform cross correlation block diagram [9].

Despite the increase in noise due to the use of the FFT to computer the correlation function, very little concern is apparent in the literature with not much mention of such limitations of the FFT technique. However, Pust (2000) [11] has studied the relative level of error generated by using the FFT compared to direct calculation of the correlation plane, 
determining that the error has actually been underestimated. It was shown that for identical input image pairs, the FFT algorithm performed poorer than did the direct computational technique for correlation plane realization. It was postulated that the peak locking problem, associated with the inability to achieve sub-pixel resolution in the correlation plane, was not only due to small particle image size but also an artifact of the FFT process itself. According to Pust, the only advantage that the FFT technique provides is an increase in computational speed but that this advantage does not outweigh the cost in accuracy.

\subsubsection{Window and Filter Functions to Compliment the FFT Cross- Correlation}

The use of a FFT to translate interrogation region intensity profiles into the spatial frequency domain results in much faster calculation of the correlation plane. However, due to the assumption that the inputs are cyclical in nature, phantom particles and phantom correlations arise as shown in Figure 2.15. A particle will tend to correlate properly with its shifted representation in the second exposure, but also will correlate with its shifted representation in the adjacent replicas of the second exposure as a result of the forced periodicity. This can be overcome by implementing a windowing function which manipulates the CCD image's pixel values to be multiplied by a factor between 0 and 1 depending where the pixel is located in the interrogation region with respect to the window. In other words, a window function acts as an input filter for the FFT algorithm. The form of the window function determines the factors, by which the pixel is multiplied, with respect to the location in the interrogation region. The windowing function is given by:

$$
W(m, n)=\left\{\begin{array}{l}
1 \text { when }\left\{\begin{array}{l}
\frac{-k M}{2} \leq m \leq \frac{k M}{2} \quad \text { and } \\
\frac{-k N}{2} \leq n \leq \frac{k N}{2}
\end{array}\right. \\
0 \quad \text { otherwise }
\end{array}\right.
$$

where $(M, N)$ is the size of the interrogation region and $k$ is a variable $(0.0 ; k \leq 1.0)$ which determines the size of the cut-off window. The smaller the value of $k$, the smaller 
the window. This effectively limits the maximum displacement of particles within the interrogation region, as noted before, to about one-quarter the width $\left(N_{\text {int }} / 2\right)$.
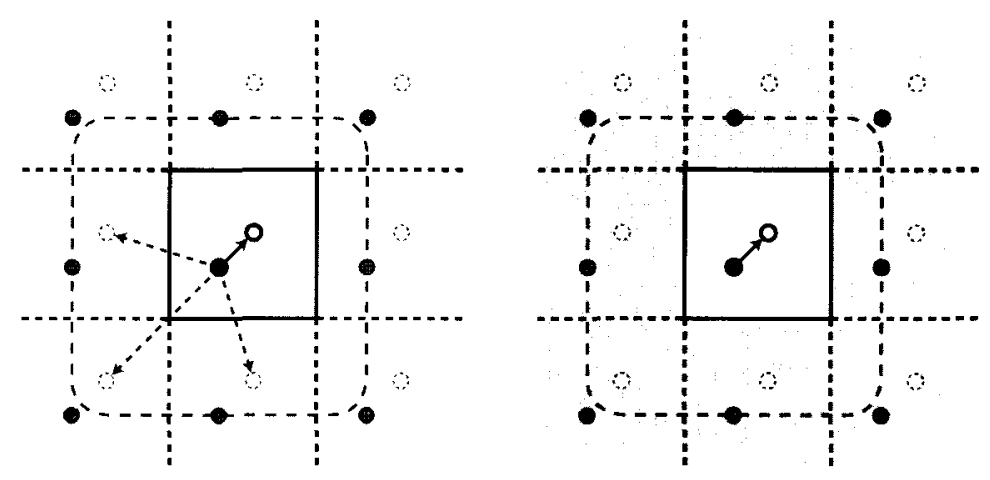

Figure 2.15: Phantom particles and correlations on the left are eliminated by implementation of the top-hat window function, shown on the right [14].

However, as was mentioned previously, discontinuities will tend to add noise into the FFT cross-correlation method, so a top-hat method is not always the best choice for a window function. A Gaussian window function acts in the same manner as the top-hat window function, but the side profile of the function is a Gaussian curve instead of a step plus a backwards-facing step, eliminating the discontinuity with a smooth transition from 1 to 0.

While the windowing functions act as an input filter to the FFT procedure, preparing the image interrogation regions to ensure the FFT identifies the displacement with as much accuracy possible, the filter functions act as an output filter. The filter functions are applied in the frequency domain removing noise in the correlation plane by eliminating spatial frequencies that do not represent physical displacements of particles. The No-DC filter removes the DC component present in the frequency domain representation of the correlation plane, which generally corresponds to background light in the images and distortions due to the optics and camera. Removing this lower frequency band removes the noise bed from the correlation plane, improving the signal-to-noise ratio.

A low-pass filter can also be applied to remove the high frequency components in the spatial frequency domain. The removal of the upper frequency band can widen peaks in the correlation plane, reducing the peak-locking effect by increasing the number of data points 
in the correlation plane surrounding and belonging to the peak. A band-bass filter may also be implemented, which is a combination of the No-DC (effectively a high-pass filter) and low-pass filter to gain the benefits of each. An example of an implementation of the band-pass filter is shown in Equation 2.24:

$$
W(m, n)=\left\{\begin{array}{ll}
4 r & \text { for } \quad r \leq \frac{1}{4} \\
1 & \text { for } \quad \frac{1}{4}<r \leq \frac{3}{4} \\
4-4 r & \text { for } \quad \frac{3}{4}<r \leq 1 \\
0 & \text { for } \quad r>1
\end{array} \text { where } r=\sqrt{\left(\frac{2 u}{U}\right)^{2}+\left(\frac{2 v}{V}\right)^{2}}\right.
$$

where $(u, v)$ and $(U, V)$ describe the coordinates in the spatial frequency domain and the size of the spatial frequency domain respectively.

\subsubsection{Discrete Interrogation Window Overlap and Offset}

The resolution of the calculated vector map can be increased drastically by overlapping the interrogation windows in the cross-correlation process. Also, since windowing functions have the negative side effect of discarding information near the edges of the interrogation region, the overlapping of neighbouring interrogation region allows the information held in these discarded areas to be used.

In general, the interrogation regions in the first and second image, compared in the crosscorrelation process, are located at the exact same location on the image plane. However, if the second interrogation region is offset, or located elsewhere nearby on the image plane, a greater dynamic velocity range can be achieved if this new location is closer to the location of the shifted particles. This point is illustrated in Figure 2.16. It follows that by placing the second interrogation region as close to the area where it will likely correlate the best with the first interrogation region, better signal-to-noise ratios can be achieved in the correlation plane [15] since correlations further from the centre of the correlation plane are less sensitive. An example of this is, given the known velocity of a flow, interrogation regions shifted 
forward in the second image by the displacement that would arise due to that know velocity. In this case, the particles in the first image's interrogation region should be nearly center on the second image's shifted interrogation region. This results in more particle image pairs being present in both interrogation regions, leading to more true correlations and thus less signal noise in the correlation plane.

First interrogation area

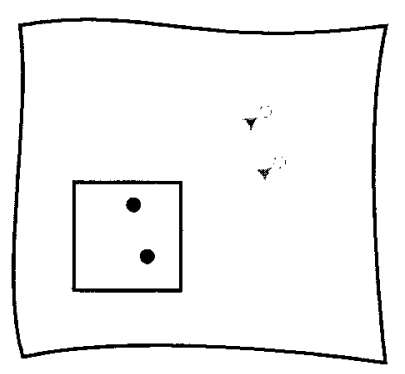

$t=t_{0}$
Second, offset interrogation area

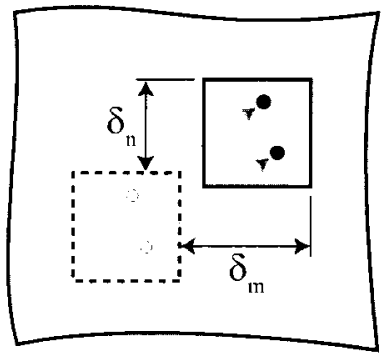

$\mathrm{t}=\mathrm{t}_{0}+\Delta \mathrm{t}$

Figure 2.16: The interrogation window in the first image on the right is correlated with an $\delta_{m}, \delta_{n}$ offset interrogation region in the second image on the left, such that the shifted particles are within the second interrogation area.

\subsection{Particle Image Velocimetry Image Post-Processing Methods}

Once the necessary calculations have been performed on the images, the resulting raw vector map must be post-processed by applying various techniques in order to arrive at a more accurate depiction of the flow being measured. Raw vector maps will often have numerous vectors which do not represent the flow being investigated, for reasons ranging from improper local seeding distribution, improper local lighting, phantom correlations and others. These vectors are often labeled as outliers or spurious vectors. It is in the interest of accuracy that the raw vector map should be subjected to a validation process to determine whether or not the vectors actually describe the flow being investigated. If they are deemed outliers, or spurious, they should be rejected as they describe some other property of the measurement technique which is not of interest. An approach to dealing with invalidated 
vectors is to replace them with an average of the surrounding vectors.

The post-processing of PIV images also include the rendering of contour plots describing the scalar velocities, pressure distribution, turbulence levels and vorticity in the measurement plane.

\subsubsection{Validation Techniques: Peak Validation}

One such technique involves the removal of spurious vectors (unrealistic vectors due to direction or magnitude) which came about due to mismatches of particles in the crosscorrelation process. This can be done by removing those vectors which have a very weak correlation coefficient, when compared to the second peak's correlation coefficient in the correlation plane. This is referred to as peak validation. Figure 2.17 shows an example of the peak validation process. The largest peak in the correlation plane is compared to the second largest peak and if the signal-to-noise ratio (ratio of the two peak heights) is less than a certain threshold ( $k$, as given in Equation 2.25), the vector is rejected.

$$
\frac{\text { Highest peak }}{\text { Second highest peak }} \geq k
$$

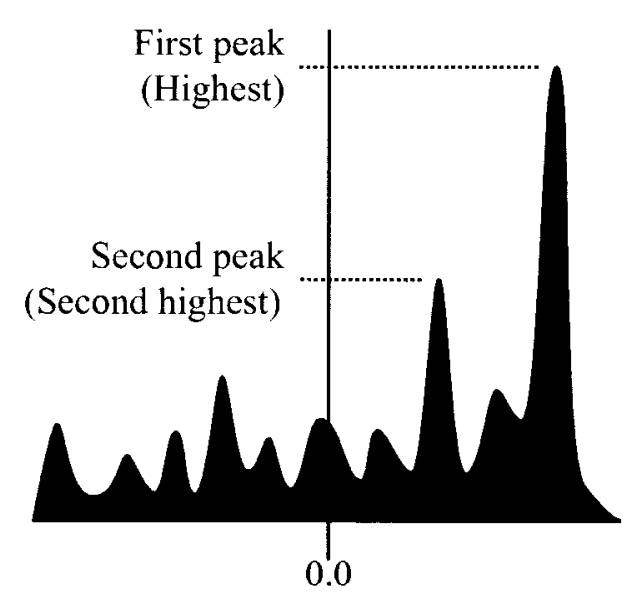

Figure 2.17: One-dimensional slice of the cross-correlation plane showing the largest peak (hopefully the signal) and the second largest peak (noise) [14]. 


\subsubsection{Validation Techniques: Range Validation}

In general, the limits of the velocities present in the flow are known. These limits can be used to isolate vectors which well exceed the upper velocity limit, or are too slow to be an actualization of the flow. This validation tool can also be used in conjunction with the constraint on the upper acceptable measurable velocity given by the interrogation region size selected. Since the particles must travel less than one-quarter of the length of an interrogation region side, and given the $\Delta t$ of the measurement, one can calculate the maximum allowable velocity. If this velocity is exceeded, the measurement likely cannot be trusted, it should be rejected and a smaller $\Delta t$ chosen if the problem is widespread. The vector is discarded, unless it satisfies Equations 2.26, 2.27 and 2.28:

$$
\text { Minimum speed } \leq\|\mathbf{V}\| \leq \text { Maximum speed }
$$

where $\|\mathbf{V}\|=\sqrt{V_{x}^{2}+V_{y}^{2}}$ is the length (speed) of the velocity vector.

$$
\begin{aligned}
& \text { Minimum } V_{x} \leq\left\|V_{x}\right\| \leq \text { Maximum } V_{x} \\
& \text { Minimum } V_{y} \leq\left\|V_{y}\right\| \leq \operatorname{Maximum} V_{y}
\end{aligned}
$$

\subsubsection{Validation Techniques: Moving Average}

A property of non-compressible flows is the absence of massive discontinuities, a property which can be forced in the measurements by the application of an averaging to ensure smooth continuity between vector locations. For the moving average validation technique, the vector's average velocity are compared to an average of all their neighbouring vectors and rejected of they differ by a certain threshold, $k$. Consider the point $(x, y)$ in the vector domain. The average of the neighbourhood centred around $(x, y)$ is calculated using 
Equation 2.29 as such:

$$
\overline{\mathbf{V}}(x, y)=\frac{1}{m n} \sum_{i=x-\frac{m-1}{2}}^{x+\frac{m-1}{2}} \sum_{i=x-\frac{n-1}{2}}^{y+\frac{n-1}{2}} \mathbf{V}(i, j)
$$

and the vector is rejected if:

$$
\|\mathbf{V}(x, y)-\overline{\mathbf{V}}(x, y)\|>k
$$

where $\mathrm{k}$ is given by Equation 2.32 and the norm $\|\cdot\|$ is defined by:

$$
\|\mathbf{V}(x, y)-\overline{\mathbf{V}}(x, y)\|=\sqrt{\left(V(x, y)_{x}-\bar{V}(x, y)_{x}\right)^{2}+\left(V(x, y)_{y}-\bar{V}(x, y)_{y}\right)^{2}}
$$

Given the idea that the vector field should be smoothly changing (no discontinuities in subsonic flows) then any one vector that deviates too much from its neighbours must be an outlier.

$$
k=k_{\alpha} \max _{x, y}\|\mathbf{V}(x, y)-\overline{\mathbf{V}}(x, y)\|
$$

where $k_{\alpha}$ is a user defined threshold, called the acceptance factor, between 0 and 1 with $k_{\alpha}=0$ rejecting all vectors and $k_{\alpha}=1$ accepting all vectors. A good ad-hoc choice for an acceptance factor is between $0.01-0.1$, but some trial and error is necessary. The factor becomes less important if a substitution is made for invalidated vectors as any vectors incorrectly identified as outliers will be replaced by vectors very similar to the original vector.

\subsubsection{Validation Techniques: Masking}

If there are objects in the flow field which is being measured, there are bound to be areas where no measurement is desired-either within the object, where velocities are meaningless or in the shadows of the objects where the laser pulse was unable to illuminate, velocities calculated through the cross correlation technique will not be valid. A mask can be created and applied to the raw vector map in order to invalidate all vectors which may appear in such areas that should not contain vector information. 


\subsubsection{Filtering: Average Filter}

Averaging methods are also generally applied, where each vector in the vector map is replaced by the uniformly weighted average of itself and all vectors within in a $(M \times N)$ sized neighbourhood centred around it. This can also be the method of choice for replacing vectors that have been deemed spurious or otherwise invalid. The formula used to calculate the average is the same as used in Equation 2.29. Only grid points with valid vectors will be used to calculate the average.

\subsection{Accuracy Improving Methods for Particle Image Ve- locimetry}

The accuracy of the data retrieved from the particle image velocimetry measurement technique is difficult to analyze and, in the literature, methods which attempt to quantify or increase the accuracy of such measurements are generally investigated using synthetic PIV images along with real PIV images validated using using Monte-Carlo simulations.

Much of the uncertainty in PIV measurements arise from sample volume size, finite tracer particle numbers and image resolution. Hart (2000) [16] developed a correlationside technique which attempts to eliminate errors in the correlation plane before the peak position is measured. This allows for a much more accurate vector field to be produced and solves problems which post-processing methods cannot solve due to the loss of information once the correlation plane is converted to a vector. By sub-correlating each interrogation region's correlation plane with its neighbours' correlation planes, the true correlations can be identified and the stochastic correlations discarded.

Due to the discrete nature of the interrogation regions, particles which end up partially inside the interrogation region region of the first or second image, contribute to noise in the correlation plane. This error has been called the partial image error (PIE) and discussed by Anandarajah et. al (2006) [17]. It has been shown that PIE has a significant effect when using interrogation regions of $32 \mathrm{px} \times 32 \mathrm{px}$ or smaller. Using a normalized signal strength 
(NSS) correlation peak prior to the sub-pixel interpolation is suggested. In addition to reducing the noise in the correlation plane due to PIE, a NSS correlation peak was shown to increase the dynamic velocity range of the measurement as well.

The turbulent nature of many interesting flows is a source of error in PIV measurements of such flows. The raw data may hold this turbulent information, but when validation techniques, such as the so called median test [18], are invoked to separate true data from the spurious vectors or outliers, the method cannot differentiate a turbulent flow from one with numerous slightly spurious vectors. In order to regain the benefit of the vector validation technique, Westerweel and Scarano (2005) [19] proposed a modification to the standard median test. The problem arises since the threshold value, which is used to determine whether or not a vector's deviation from its neighbours' is enough to be called an outlier, is constant. Thus, the method cannot differentiate between a valid, physical fluctuation $\left(u^{\prime} o r v^{\prime}\right)$ as a manifestation of turbulence and a spurious vector due to signal noise. By weighting the threshold value with an estimated expected $\left\|\left(u^{\prime}\right)^{2}+\left(v^{\prime}\right)^{2}\right\|$, evaluated using the set of close neighbours, this differentiation can be made.

Since the fluid cells within a flow tend not to remain square, the estimation of such flows by using square (or any fixed sided geometry) interrogation regions can lead to uncertainty, depending on the flow being investigated. This uncertainty was quantitatively derived and identified by Huang et. al. (1993a) [20] and a remedy suggested (Huang et. al. (1993b) [21]). The suggested modification allowed for deformable interrogation regions, the exact deformation of which would be calculated using a priori knowledge of the flow, estimated using conventional PIV techniques. The conventional interrogation regions would then be replaced with slightly larger (still square) interrogation regions which encompass the newly determined, deformed interrogation region. This method requires a relatively substantial computational effort and relies on the estimate of the flow field to provide enough successful indicators of the flow such that a valid deformation can be generated.

To increase the likelihood that groups of poor data in PIV measurements are removed together, Nogueira et. al. (1997) [22] developed a validation method based on the identification of coherent vector zones within the vector field. The intelligent algorithm, capable of 
identifying regions of similarity, validates the large coherent zones and discards very small collections of like vectors, including lone vectors, that are deemed spurious. The method intends to mimic the human minds identification of spurious vectors by first identifying regions of coherent vectors. An interpolation technique was developed to refill the zones which were discarded due to being labeled spurious. The missing zones were interpolated from the surrounding zones that were considered valid.

\subsubsection{Recursive Local-Correlation}

In order to increase the overall resolution and accuracy of the cross-correlation technique, iterative local-correlation methods have been developed which utilize the displacement vector map as a guide for further repeated cross-correlation calculations on the same image pair. Scarano et. al. (1999) [23] and Hart (1999) [24], for example, have verified the use of such techniques which will be described below. These methods were based on experimental methods of Keane and Adrian (1993) [25] and Keane et. al. (1995) [26] which were adaptations of suggestions made previously by Perkins and Hunt (1989) [27] and Guezennec and Kiritsis (1990) [28] on the topic of super-resolution PIV.

The technique begins by performing a standard cross-correlation on the image pair, using rather large interrogation windows (128 px $\times 128 \mathrm{px}$ or larger). This results in a relatively accurate (due to large interrogation window size), full vector map of displacements (velocity not yet calculated) in pixels. The vector map of pixel displacements is then validated with the techniques described earlier, using neighbouring pixel displacements. The image pair is then re-evaluated using slightly smaller interrogation regions, usually halved in each direction as a result of the requirement of the FFT method, but any reduction in size is theoretically possible. This time, however, the interrogation region offset technique is used, with an offset based on the previously calculated displacements of the larger interrogation region within which the current, size-reduced cell is in. The vector map is then validated and the second iteration is complete. This process can be repeated numerous times, until the interrogation region is sufficiently small that further reduction becomes impossible due to the initial image's resolution. 
A further enhancement in accuracy can be made by introducing an iterative validation process between subsequent interrogation region size reducing iterations, as developed by Wenguo et. al. (2001) [29]. Immediately after the vector validation, during one of the outer iterations described earlier, the calculated preliminary vector map is re-evaluated without modifying the interrogation region size. During this re-evaluation, a validation method using the calculated displacements as an interrogation region offset is employed, and a further increased reliability of the next iteration's offset can be achieved. This subiteration can be performed numerous times, each time refining the offset vector map of the previous iteration. Therefore, by adding a set of validating sub-iterations to each of the outer interrogation region size-reducing iterations, the accuracy of the vector maps calculated at each outer iteration will higher and thus result in a better estimate of the next iteration's offset for each of the new smaller interrogation regions. An illustrated flowchart of the recursive local correlation process with the optional refinement step, can be seen in Figure 2.18 .

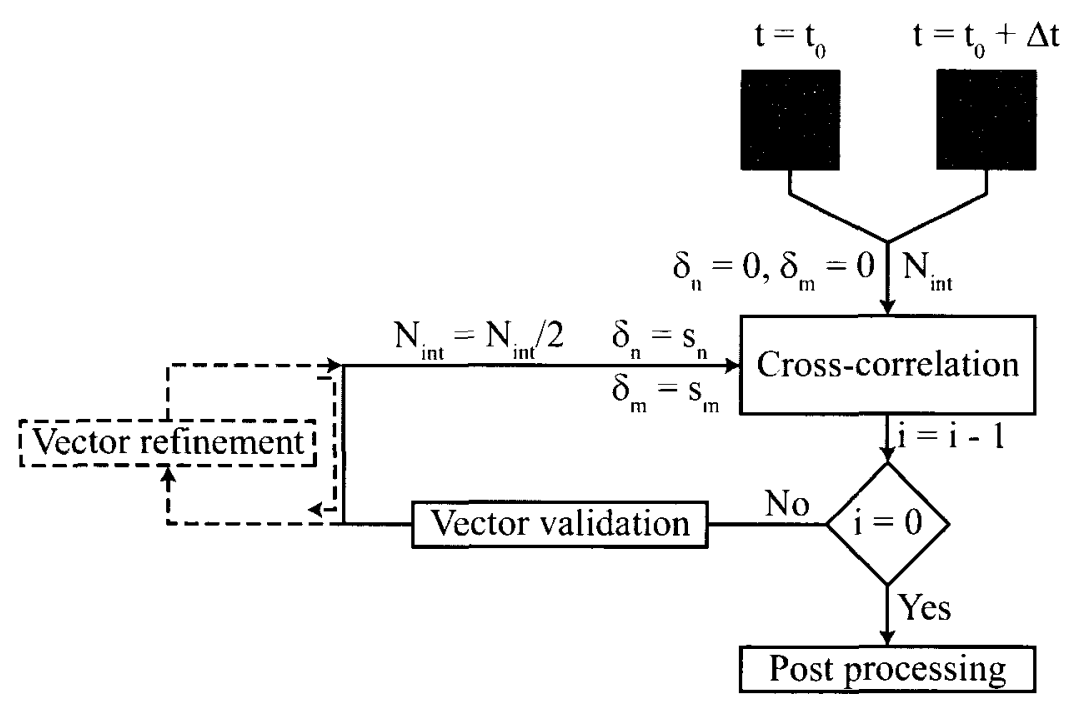

Figure 2.18: Flowchart describing the iterative local-correlation method used to gain super resolution of PIV measurements with an optional recursive inter-iteration validation scheme.

In Figure 2.18 a flowchart of the entire possible iterative method is shown. For a measurement image pair, regular cross-correlation is performed. If $i i_{0}$, where $i$ is the user 
selected number of iterations to be performed (depending on initial interrogation region size and desired final interrogation region size), then the raw displacement vector plot is validated, saved and carried forward for use during the next iteration. At this point, a vector refinement scheme can be implemented as described earlier. The displacement vector map, whether it was refined or not, is then used as the offset values for the next cross-correlation pass on the same two initial images. This time, however, the interrogation size is reduced and, as mentioned, an offset value is now chosen for each interrogation region. In general, the dimensions of the interrogation regions are halved, resulting in a four-fold increase in the number of interrogation cells. The four new cells created in each of the previously used interrogation regions are assigned an offset that corresponds to that original cells displacement, found during the previous cross-correlation. The new, four times as dense grid of offset interrogation regions is applied to the original two images, then cross-correlated once again. This process is repeated until $i=0$.

This powerful technique used in conjunction with interrogation overlap can produce a very reliable and dense set of velocity vectors which have been shown to improved the dynamic velocity range and spatial resolution [30] when compared to the generic crosscorrelation method. When compared to the general, fixed interrogation window method, the recursive local-correlation window shifting algorithm has shown to increase accuracy by up to $50 \%$ [31]. The increased amount of required computational power and time is the downside to this technique, but the gain in resolution and accuracy certainly outweigh this fault.

\subsection{Particle Image Velocimetry Measurement Error Analysis}

The overall uncertainty in a PIV measurement is generally composed of the uncertainty in the ability of the tracer particle to accurately follow the flow and the uncertainty related to the accuracy to which the PIV technique can extract velocity information from the image pairs or tracer particles in the flow. The tracer particle's ability to follow the flow was discussed earlier, and with small enough particles it can be assumed that the particles follow 
the flow close enough that any slight deviation will not likely be larger than the resolution of the measurement itself and thus negligible. The PIV image analysis technique, however, is subject to a few error-contributing factors, some of which depend on seeding characteristics.

Through the use of synthetic images, created by specifying various parameters including the image contrast, image noise, particle density, particle image diameter, and particle displacement between frames, Guezennec and Kiritsis (1990) [28] quantified the error due to variance in these parameters. This statistical analysis of artificially generated PIV images showed that, in most cases, image contrast was the most important contributor of correlation-side error. It was determined that, with sufficient contrast in the PIV images and the use of a few aforementioned accuracy improving techniques, the error in the measured particle displacements was on the order of a few percent, when compared to the known displacements in the synthetic images.

The use of synthetic images provides useful information about the accuracy of the PIV measurements due to the a priori knowledge of the particle displacements. However, as shown by Westerweel (2000) [32], if the synthetic tracer particle ensemble does not correctly represent the probability density function of a real image intensity, the results will systematically underestimate the error of the technique. This was done via comparison to a Monte-Carlo simulation.

Using the appropriate synthetic image generation methods, Westerweel (2000) [32] determined that the minimum achievable error for sub-pixel displacement estimations is about $0.02 \mathrm{px}$ and can only be achieved with particle images larger than $2 \mathrm{px}$. This lower bound was also found using particle ensembles of identical particle sizes, something which is not routinely achieved in practical PIV use, but could be achieved using monodisperse particle generation. It was determined that $25 \%$ variance in the particle size could easily increase the error by a factor of $2-4$. 


\section{Chapter 3}

\section{Blade (Airfoil)-Vortex Interaction}

Since the investigation in this study is completely two-dimensional, it is proper to refer to the blade-vortex interactions studied here as airfoil-vortex interactions. When speaking of the two-dimensional component of the fully three-dimensional phenomenon, it will be referred to as such from this point forward.

The vortices which play the lead role in BVI are produced as a wing (or blade) tip vortex, which is a natural consequence of the pressure differential, characteristic of any aerodynamic lifting body. The rotor blades of a helicopter leave behind a continuous vortex sheet which rolls up into two distinct vortices (see Figure 3.1). The locations at which the sheet rolls up to form these single coherent trailing vortex lines are at the hub and tip edges of the rotor blade. The hub vortex is quickly pushed beneath the rotorcraft due to the strong downwash and becomes relatively well-removed from the blade path [33]. This part of the rotor vortex sheet does not have a significant influence on the blade-vortex interaction that is the topis of this thesis.

The tip vortex, however, remains present within the aerodynamic area of influence of the main rotors. This continuous vortex line, which is traced out by the blade tip as the rotor makes its circular path (relative to the hub), may interact with the remaining blades over different stages of the rotation depending upon the type of movement the rotorcraft is executing. Figure 3.2 shows a plan view of the traced out path of the tip vortices and the different locations where BVI will occur for a typical two-blade rotorcraft during descending-forward flight. 


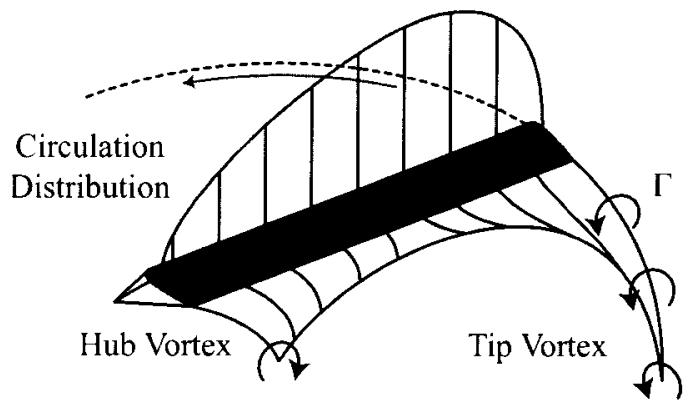

Figure 3.1: Creation of the tip vortex as a result of bound circulation $\Gamma$ about the rotor blade [34].

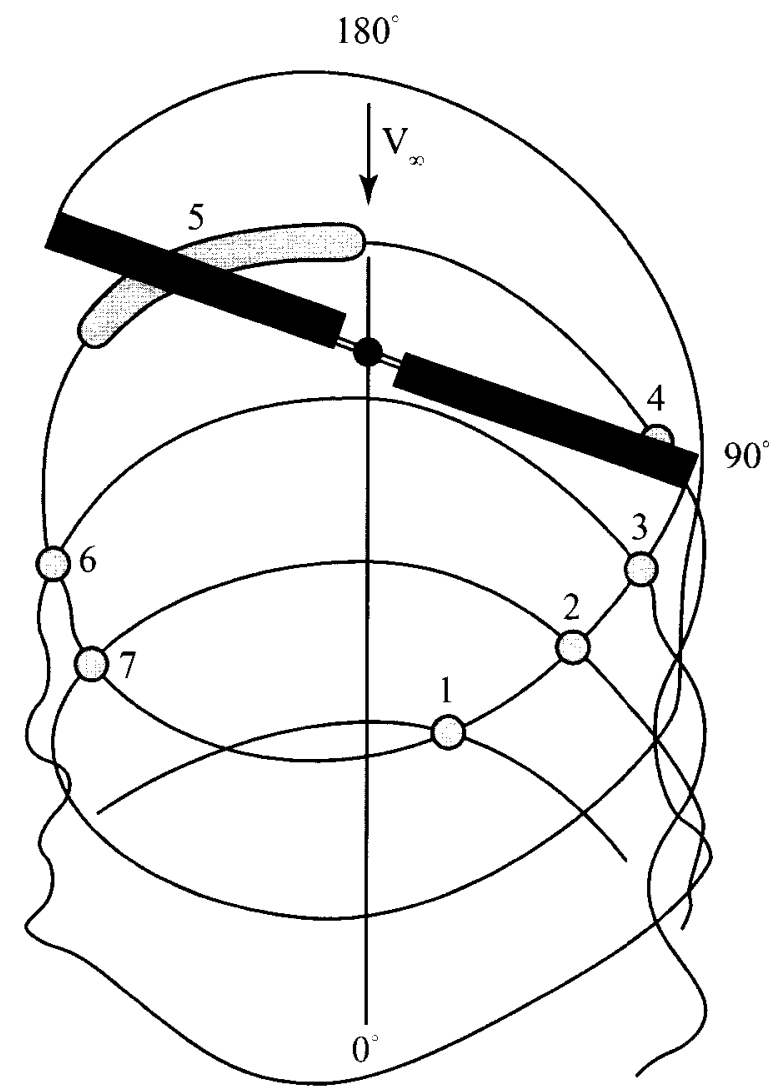

Figure 3.2: Blade-vortex interactions during descending flight of a typical two-blade helicopter (7 blade-vortex interactions labeled) [35].

As mentioned earlier, blade-vortex interactions occur mainly in part due to the confined airspace where the helicopters's rotor blades operate and their repeated motion through this confined airspace which has been contaminated by the rotor wake. The amount by which the rotors and the rotor wake overlap is heavily dependent on the direction and magnitude 
of the free stream velocity-the specific type of motion the rotorcraft is performing. During heavy ascent, for example, the rotors are less likely to encounter tip vortices within the rotor wake due to the additive nature of the downwash and free stream velocities in this case. However, during other motions such as descent, these two velocity components will interfere destructively. Tip vortex lines will cross paths with trailing blades multiple times at multiple locations, resulting in undesirable effects of these interactions.

The most significant effects of BVI are observed mainly during descending flight, up to moderate advance ratios, and rotorcraft manoeuvres. It is during these flight conditions where the tip-vortex and rotor blade paths intersect most closely (see Figure 3.3). As the rotorcraft travels forward and level, the helical vortex structures tend to flow downward and out of the rotor's path due to the downwash of the main rotor. In descending forward flight, however, the downwash is counteracted by the free stream velocity and causes the vortex rings to pass directly through the main rotor's path. This results in blade-vortex interactions, of which there are seven significant interactions on the advancing and retreating side of the rotorcraft for moderate advancing ratios (velocity dependent). Figure 3.2 shows the seven locations of the various significant BVI events, on both the retreating and advancing side, through one complete revolution of the helicopter's rotor during descending flight. Locations 1 through 4 indicate the interactions on the advancing side and locations 5 through 7 indicate ones occurring on the retreating side.

It has been observed that the retreating-side BVI events are less intense than those which occur on the advancing side. This can be attributed to the simple fact that the advancing side interactions occur at a much higher Mach number due to the additive-free stream and blade's radial velocities. The advancing side interactions, which also happen to be the more intense far-field noise producing interactions [7,37], are depicted individually in Figure 3.5 with their appropriate names included. Of the advancing side interaction types, the $\gamma$ type BVI is the most intense [37], due to the alignment of the blade axis with that of the interacting vortex axis. Furthermore, it turns out that only those blade-vortex interactions where the angle between the blade axis and the vortex axis, $\gamma$, is zero or near zero provide any significant contribution to the far field noise. The mechanics which are 


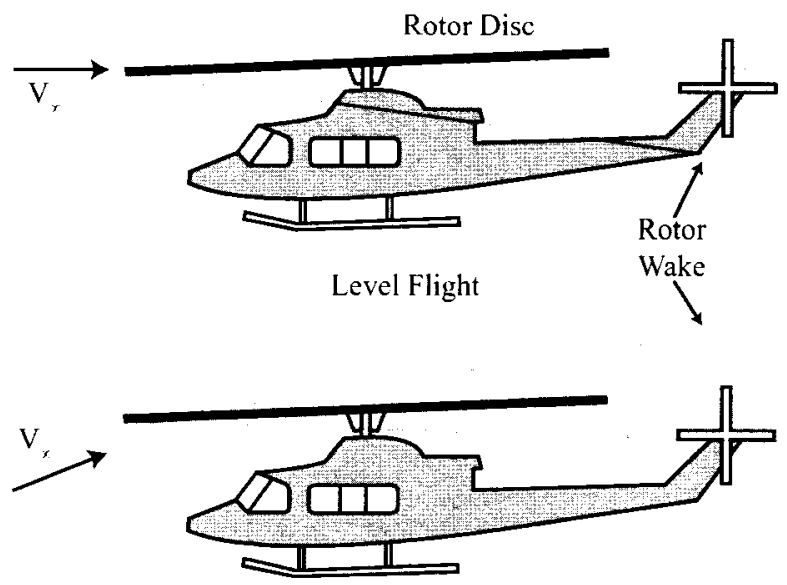

Descending Flight

Figure 3.3: The effect which a descending flight path has on the interaction between the rotor wake and the rotor blades compared to level flight [36].

responsible for this are related to BVI acoustic phasing and will be explained in section 3.1 in further detail.

The two-dimensional representation of a fully three-dimensional blade-vortex interaction provides very useful information concerning the mechanisms involved in the transfer of energy from the vortex to the blade, including the resulting vibration and noise. Thus, although a purely AVI investigation cannot completely describe the realistic counterpart, BVI, it serves as a valuable tool for investigating this phenomenon due to the ease at which otherwise complex and nearly impossible measurements can be made. As will be shown later in this chapter, when the vortex axis is parallel to the blades axis, the two-dimensional representation, AVI, provides a quite accurate [6] description of the aerodynamics within the plane perpendicular to the blade and vortex axes during the BVI event. Conveniently, this specific orientation of the blade and vortex axes also results in the most intense form of the BVI effect in rotorcraft, and therefore the most interesting form from the perspective of noise and fatigue. Thus, the limit where the angle between the vortex and rotor blade axis is zero $\left(\gamma=0^{\circ}\right)$ was the specific type of BVI which was the focus of this study. 


\subsection{Mechanics of the Blade-Vortex Interaction}

As was previously mentioned, a critical source of rotorcraft noise and vibration is caused by the blade-vortex interactions which occur during various flight manoeuvres of a helicopter. Depending on the orientation of the vortex axis and the blades axis when the two collide, the magnitudes of the effects of this interaction vary. A useful parameter for measuring the blade-vortex interaction's effect is the Mach trace number, as given in Equation 3.1.

$$
M_{t r}=\frac{\Omega r_{b}+V_{\infty} \sin \psi_{b}}{a_{0} \sin \gamma}
$$

The Mach trace number describes the local Mach number experienced by the blade during the blade-vortex interaction, and depends on the angle at which the rotor blade axis makes with the vortex axis $(\gamma)$, the azimuth angle of the blade $\left(\psi_{b}\right)$ relative to the free stream velocity $\left(V_{\infty}\right)$, the speed of sound $\left(a_{0}\right)$ and the velocity of the point on the blade where the interaction with the vortex occurs $\left(\Omega r_{b}\right)$. It also provides useful information on the directionality of the resulting radiated noise [38].

A concept which is useful for the understanding of BVI mechanics is the phase emissions of BVI acoustical waves along the portion of the tip vortex that has interacted with the rotor blade. Figure 3.4 illustrates the acoustic BVI phasing concept with a single rotor blade intersecting with a tip vortex path. The BVI is often assumed to be chordwise compact and the integrated effect acts at the quarter-chord of the airfoil [7]. Thus, since the acoustic reaction radiates outward spherically at each point along the intersecting path through time, certain areas in the near and far fields are bound to experience constructive interference of the temporally and spatially separated wavelets emanating from the intersection of the quarter chord line of the blade and vortex axis. This undesirable wave amplification results in the loud 'popping' sound that is characteristic of BVI noise. As the reader can see, considering the BVI acoustic radiation as a series of out-of-phase spherical wavelets is a powerful tool in the prediction of BVI noise.

Figure 3.5 describes the four types [39] of blade-vortex interactions which occur on the 


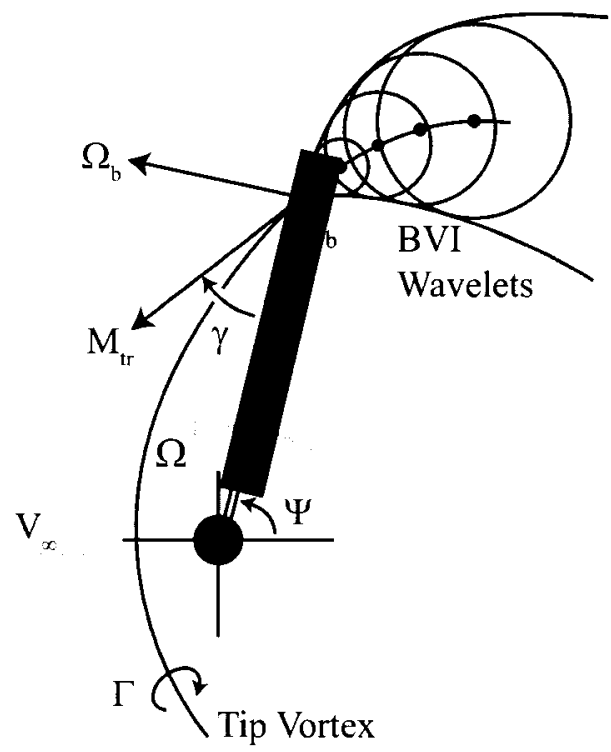

(a) Advancing Side

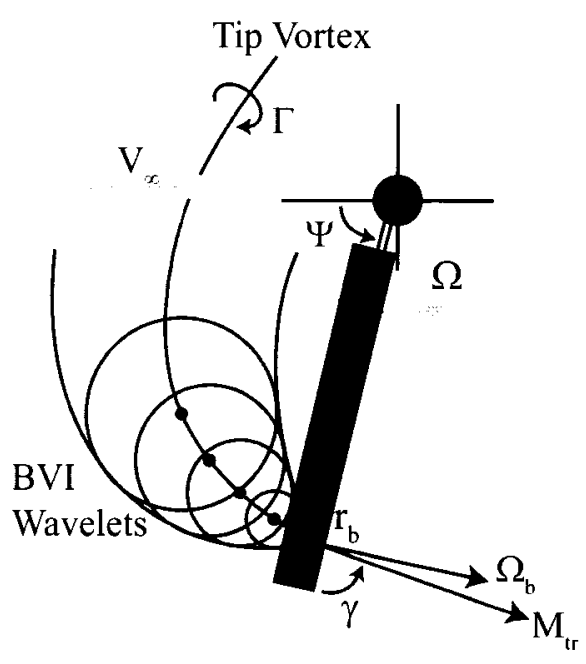

(b) Retreating Side

Figure 3.4: Phase emissions of blade-vortex interaction waves in a single blade and tip vortex interaction [7].

advancing side, as well as the Mach trace number $\left(M_{t r}\right)$ along the non-dimensional blade radius during each type of interaction. The $\alpha$-type interaction experiences its peak effect at approximately $70^{\circ}$ azimuth and occurs mainly near the tip of the blade with little to no significant effect near the rotor hub. The $\beta$-type occurs near $55^{\circ}$ and, like the $\alpha$-type also has an effect near the tip, but accelerates to larger Mach trace values near the hub. The $\gamma$-type results in the largest Mach trace numbers and involves two blade-vortex interactions acting simultaneously at two different locations on the rotor blade. These positions achieve supersonic Mach values and rapidly accelerate together meeting near the mid-point on the blade where the angle formed by the blade and vortex axis becomes zero $\left(\gamma=0^{\circ}\right)$. It is due to this that the $\gamma$-type BVI is also referred to as parallel blade-vortex interaction. Due to the phasing of the acoustic waves from each of the two rapidly converging BVI events, the net result in the near and far-field is relatively intense. The $\gamma$-type BVI occurs at about $45^{\circ}$ azimuth. The last type of BVI on the advancing side is the $\delta$-type which generally occurs at an azimuth of less than $30^{\circ}$. This type also often reaches supersonic Mach numbers and occurs mainly near the hub of the blade. This classification of the different BVI types holds 
for all types of rotorcraft with any number of blades.

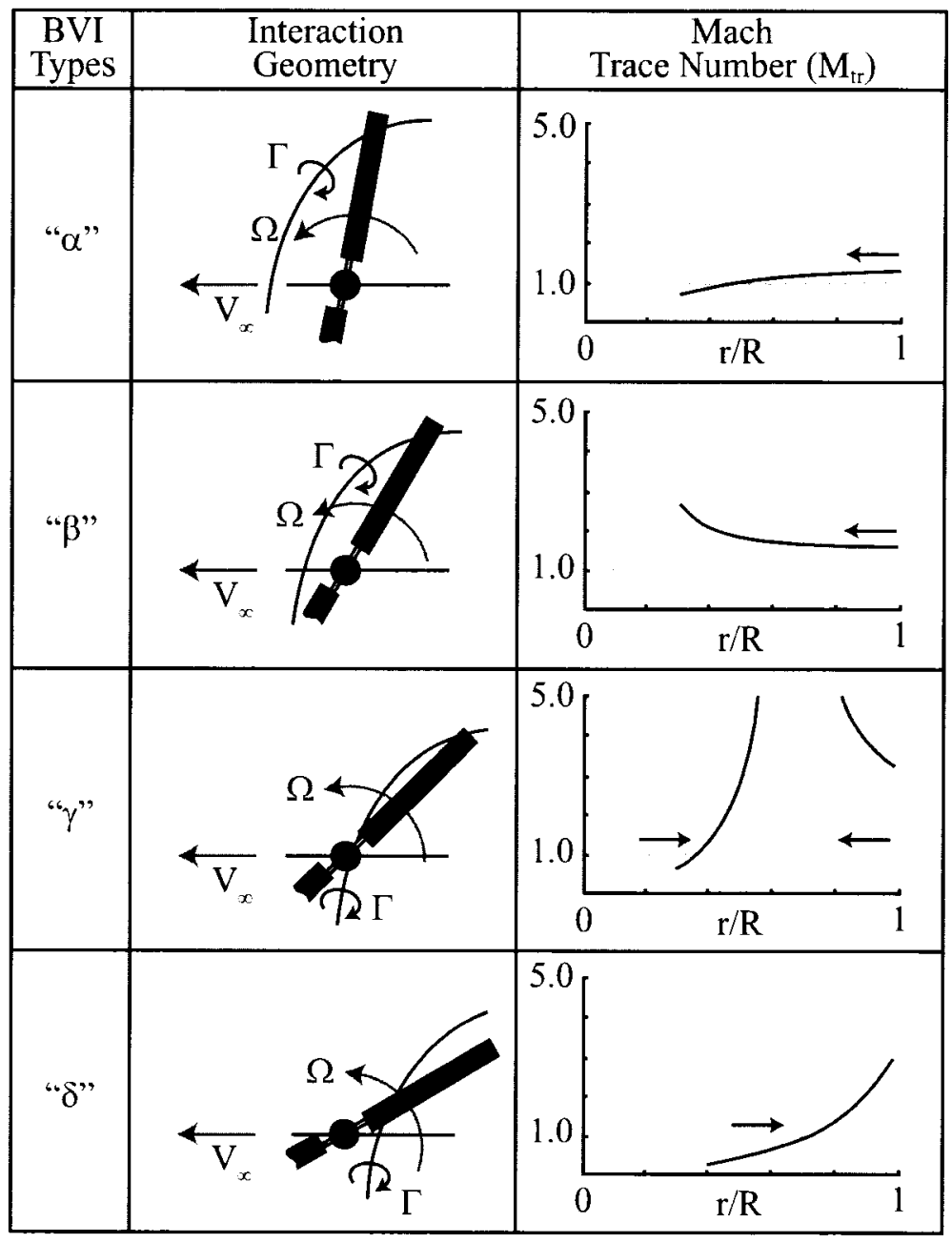

Figure 3.5: Various types of blade-vortex interactions in rotorcraft [7].

Indeed, it is apparent from Equation 3.1 that when $\gamma=0^{\circ}$ the Mach trace number theoretically becomes infinitely large. This is reflected in the $\gamma$-type section of Figure 3.5. This result, coupled with the phasing effects of the dual BVI are indicative of why this type of BVI results in the greatest noise and vibration effects.

A schematic, in plan and side view, for a typical vortex path in the parallel blade-vortex interaction is shown in Figure 3.6. The vortex miss-distance, or vertical distance from the chord line to where the vortex is located, is given by $y_{v}$. The distance that the vortex is from the nose of the airfoil is given by $x_{v}$. The circulation present in the incoming vortex 
is denoted by $\Gamma$.

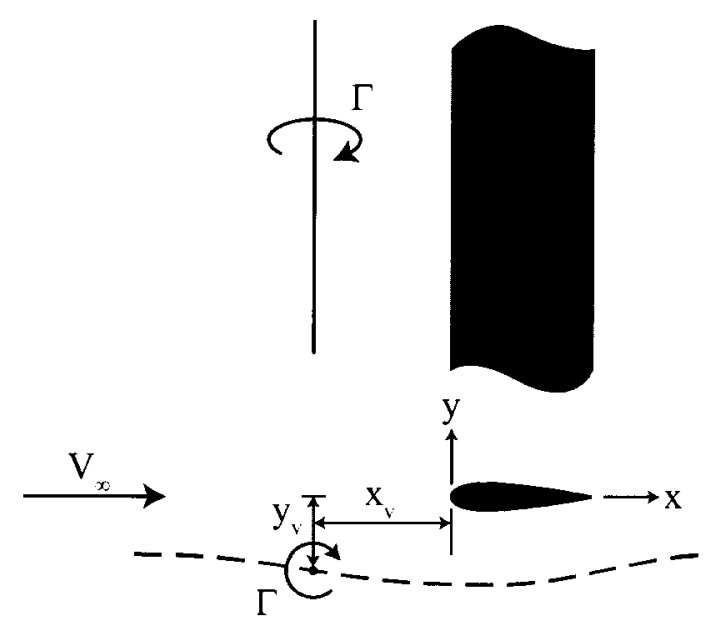

Figure 3.6: Path of a vortex with respect to the airfoil in the parallel blade-vortex interaction case $\left(\gamma=0^{\circ}\right)[6]$.

\subsection{Wind Tunnel Vortex Creation Methods}

To make a blade-vortex interaction measurement in a controlled environment, a vortex must be introduced into the flow and allowed to impact the rotor blade. This proves to be quite a challenge, especially if the goal is to introduce the vortex without otherwise disrupting the flow. There are numerous ways this has been handled in the literature, both experimentally and numerically, and the previously used methods will be further discussed later in this chapter. For this study, a few methods were chosen and implemented. These methods include the use of the starting vortex, which is created whenever there is a change in lift on a body in a flow, and also the unsteady flow in the wake of a bluff body (which also turns out to be a manifestation of the starting vortex). The theoretical background responsible for these two specific methods will be discussed briefly and their corresponding drawbacks to wind tunnel use analyzed.

Figure 3.7 shows three cases of simulated flow over a lifting airfoil with different mathematically valid solutions of circulation. Two $(3.7 \mathrm{a}, 3.7 \mathrm{~b})$ cases, although mathematically 
sound, do not satisfy the Kutta condition and one case does $(3.7 \mathrm{c})$. When the Kutta condition is satisfied, the flow on the upper and lower surfaces leave the tail of the airfoil smoothly. When this is the case, the circulation around the airfoil is equal to the Kutta circulation and simulates the flow observed in measurements. This circulation is called the bound circulation of the airfoil.

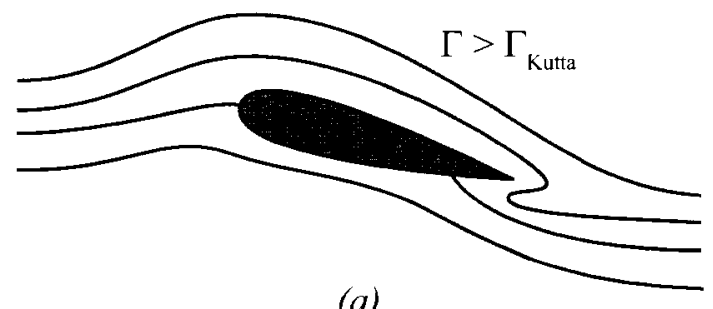

(a)

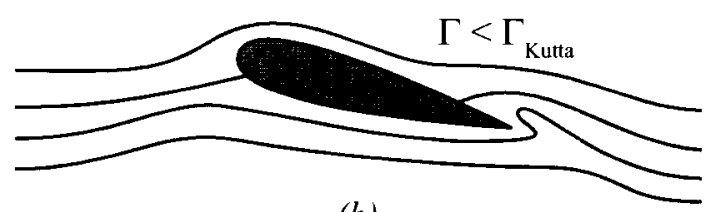

(b)

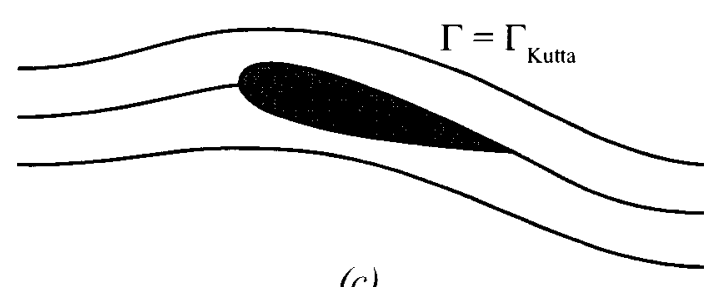

(c)

Figure 3.7: Demonstration of the Kutta condition: shows the cases where the bound circulation is greater than the Kutta circulation (a), less than the Kutta circulation (b) and the case where the Kutta condition is satisfied (c) [40].

This bound circulation is not fictitious and is a realization of the quicker fluid velocities on the upper surface of the airfoil. The actual flow around a lifting body is the superposition of the flow about the body with no bound vorticity (which would look similar to Figure 3.7b) and a vortex field with the circulation of $\Gamma_{K u t t a}$ and direction dependent on the direction of the lift (clockwise in the case of Figure 3.7). 


\subsubsection{Starting Vortex}

Since in any flow the net circulation must always remain zero, or be conserved within the flow, any bound circulation generated around the airfoil through a change in lift must be present in the flow in the form of a vortex. The result of this requirement is commonly observed during the take-off of a fixed-winged aircraft, as the buildup of circulation around the lifting bodies is shed as a starting vortex. This necessary result of any change in lift on any object can be exploited in a wind tunnel to generate a coherent vortex structure to be used in BVI studies. The relationship between the lift and the bound circulation in the flow, is given by Equation 3.2:

$$
\Gamma=\frac{L}{\rho_{f} V_{\infty}}
$$

This is the amount of circulation that is required to satisfy the Kutta condition, given a certain lifting force $(L)$ and flow velocity $\left(V_{\infty}\right)$ in a fluid of density $(\rho)$. For an lifting airfoil, the lift per unit width is given by Equation 3.3:

$$
\frac{L}{w}=l=\frac{1}{2} \rho_{f} V_{\infty}^{2} C_{L} \bar{c}
$$

where $C_{L}$ is the lift coefficient for the airfoil and $\bar{c}$ is the chord. The theoretical lift coefficient for a 4-Digit NACA airfoil is ??:

$$
C_{L, t h e o r y} \approx 2 \pi \sin \left(\alpha+\frac{2 h_{c}}{\bar{c}}\right)
$$

where $h_{c}$ is the maximum camber of the 4-Digit NACA airfoil and $\alpha$ is the angle of attack. At small angles of attack for a NACA0012 airfoil (no camber), Equation 3.4 can be approximated by Equation 3.5:

$$
C_{L}=2 \pi \sin (\alpha) \approx 0.11 \alpha
$$

where $\alpha$ is in degrees. Thus, the change in circulation per unit width of the airfoil, or amount shed in a starting vortex, upon changing either the angle of attack or the free 
stream velocity is given by Equation 3.6:

$$
\frac{\Delta \Gamma}{w} \approx 0.6 \Delta V_{\infty} \Delta \alpha \bar{c}
$$

which is valid only for small changes in angles of attack.

The impulsive pitching in one direction of an airfoil fixed at its quarter-chord point in a wind tunnel will achieve the desired result. When the airfoil is pitched through $\Delta \alpha$ degrees sufficiently fast, a starting vortex will form on the upper surface of the airfoil with circulation given by Equation 3.6. This vortex will then detach from the airfoil and flow downstream into another airfoil that is to serve as the investigation point for the ensuing BVI.

Since the pitching airfoil would have to be at about the same height in the wind tunnel as the target airfoil, the target airfoil will be positioned directly within the wake from the vortex-generating airfoil. This will certainly affect the flow at the target airfoil and therefore not perfectly mimic the flow of one isolated vortex hitting an airfoil that is desirable for BVI measurements. The amount that the wake will influence the airfoil will depend on the size of the vortex-generating airfoil, the maximum angle of attack that the airfoil experiences and the distance upstream of where the target airfoil is placed.

Another method to exploit the conservative property of circulation in order to create a vortex in a wind tunnel is to move an airfoil through the flow vertically to briefly create an effective angle of attack, then stop it suddenly. If the vortex-generating airfoil is held at the top of the wind tunnel then plunged downward, it will release a starting vortex with a circulation depending on the downward velocity. This new state will stabilize and when the airfoil is suddenly stopped again, an equal but opposite starting vortex will be shed since the lift will return to zero and the circulation $\left(\Gamma_{K u t t a}\right)$ which was present is no longer bound to the airfoil. The magnitude of the generated circulation can be calculated by using the effective angle of attack $\left(\alpha_{e}\right)$ and new free stream velocity $\left(V_{\infty}^{\prime}\right)$ which results from the vector addition of the free stream velocity and the downward velocity of the airfoil. The vortex created with this sudden change in lift due to the abrupt stop, as opposed to the 
vortex generated by the initialed motion, can be used for the BVI measurement since the location will be well known. Again, however, this will cause a large disturbance in the flow, in addition to the vortex generation, for the BVI event downstream but the disturbance, compared to the pitching type, will only be present in the flow downstream of the vortex and influence the airfoil after the BVI event takes place.

\subsection{2 von Kármán Vortices}

The oscillating vortex street observed in the wake of bluff bodies due to separation was explained theoretically by Theodore von Kármán in 1912. This repeating swirling unsteady flow was characterized by the non-dimensional parameter called the Strouhal number, given by Equation 3.7:

$$
S t=\frac{\omega d_{c}}{2 \pi V_{\infty}}
$$

where $d_{c}$ is the useful length parameter of the bluff body, such as diameter for a cylinder. The vortices are shed with the frequency $\omega$ and alternating in direction of rotation. The vortices are only observed over the Reynolds number range of $10^{2} ;$ Re $; 10^{7}$. Figure 3.8 shows a flow visualization of the von Kármán vortex street in the wake of a circular cylinder and a plot of the experiential Strouhal number versus the flow Reynolds number.

The vortex street caused by a bluff body can be used in BVI measurements as the method of creating a coherent vortex. The disadvantage of this method is that one isolated vortex cannot be achieved and the measurement must be made in the presence of the entire vortex street. However, if the streamwise separation of the vortices in the von Kármán wake is sufficient, useful measurements are possible. The ideal case where one vortex positioned in an otherwise undisturbed flow is encountered by the blade is somewhat fictional as well as ideal, since the helicopter's blades pass through numerous isolated vortices during one rotation and the fluid is anything but otherwise undisturbed. 


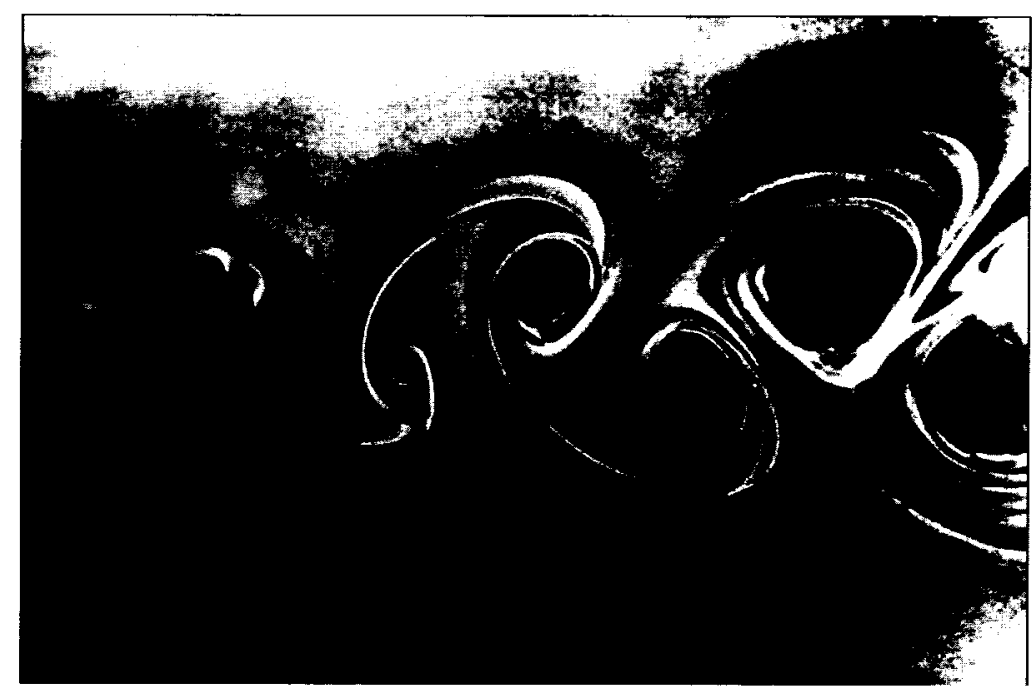

(a)

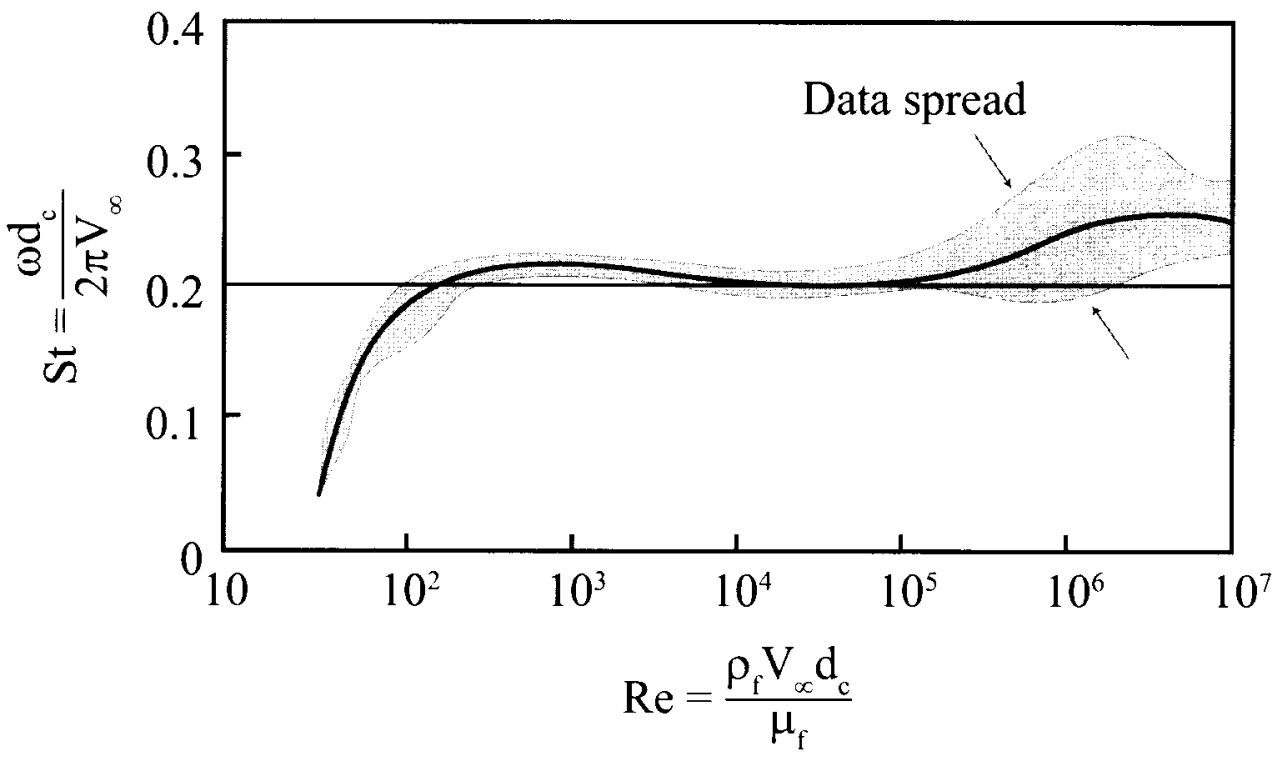

(b)

Figure 3.8: (a) A flow visualization of the von Kármán vortex street in a flow around a circular cylinder and $(b)$ the experimental shedding Strouhal number over Reynolds number [40].

\subsection{Previous Study into the Blade-Vortex Interaction}

A substantial volume of research has been directed at the noise and vibration problems created by the blade-vortex interaction in rotorcraft, as well as the mechanisms by which 
this phenomenon occurs. Likewise, much work has been done on the implementation of design-side mechanisms to alleviate the unwanted effects of BVI. Here, the current state of blade-vortex interaction research will be discussed, work employing both numerical and experimental methods, as well as an in-depth look into BVI investigations which implemented the particle image velocimetry measurement technique.

The creation of the vortex in both the numerical and experimental approaches is a challenge, and the methods used within will be analysed from an experimentalist's viewpoint. When making such measurements in a controlled environment, such as a wind tunnel or a cluster of parallel processors, it must be ensured that the interaction parameters match those observed for in-situ BVI events. This challenge provides a niche for numerical simulations, as the creation of a fictitious controllable vortex in a digital environment is a much simpler feat than creating one in a physical environment. The accuracy of the representation of the vortex in of such a simulation, however, proves to be quite another challenge, and relies on experiments to derive the specific parameters of BVI vortices.

As this current study is aimed at providing future BVI researchers with the setup and methods to make any parallel BVI measurement they wish, a careful analysis of previous work in this field is necessary to deliver such a comprehensive analysis tool. Two comprehensive reviews of the computational approach to blade-vortex interactions have been completed by Rahier (1997) [41] and Tung et. al (1996) [42].

The vast majority of both numerical and experimental investigations for the blade (or airfoil) vortex interaction have used the NACA 0012 airfoil at zero angle of attack as the test rotor, with some investigation completed at various inclined angles as well. Many of the vortex generation methods, mostly with experimental but some numerical, used NACA 0012 or NACA 0018 airfoils with a pitching or oscillating motion, or a shock tube technique. All of these methods relied on the start up vortex as a means of vortex creation.

\subsubsection{Numerical Study of Blade (Airfoil)-Vortex Interaction}

The numerical treatment of the blade-vortex interaction event varies in the literature from aerodynamic model building concepts, such as Schmitz et. al (2000) [7] (an extension of 
their own previous work [39]), and Berenger et. al (1997) [5], to thin-layer Navier-Stokes (Euler) equations $[6,36,43-47]$. In the majority of the studied literature which invoked a Navier-Stokes related solver, the chosen method of introducing the vortex into the flow was simply embedding the desired vortex velocity profile a few chord lengths upstream of the test rotor via superposition and allowing it to convect downstream. Table 3.1 shows a list of some of the previous numerical studies which have employed this method of vortex introduction and the vortex parameters used.

Table 3.1: Vortex parameters (non-dimensionalized with $\bar{c}$ and $V_{\infty}$ ) of previous BVI studies utilizing the embedding method of vortex flow introduction.

\begin{tabular}{lllll}
\hline Study & $|\Gamma|$ & $a_{0}$ & $M_{\infty}$ & $y_{v}$ \\
\hline \hline Srinivasan et. al (1986) [6] & 0.2 & 0.05 & 0.8 & -0.26 \\
Wong et. al (2000) [43] & 0.118 & 0.05 & 0.5 & -0.26 \\
Lee et. al (1994) [36] & 0.283 & 0.018 & 0.5 & $0,-0.1$ \\
Oh et. al (2002) [44] & $0.2,0.5$ & $0.05,0.125$ & 0.8 & $-0.26,-0.1$ \\
Lin et. al (1997) [46] & 0.28 & 0.018 & 0.5 & 0 \\
Lee (1994) [47] & 0.28 & 0.02 & 0.5 & 0 \\
\hline
\end{tabular}

The studies shown in Table 3.1 investigated both transonic and subsonic cases (specifically Srinivasan et. al (1986) [6]). The research by Lee et. al (1994) [36] demonstrated both numerically and experimentally that viscous effects play a significant role in parallel BVI and should not be ignored.

Some researchers (Sim et. al (2000) [7] and Schmitz et. al (2000)), as mentioned, have modelled the BVI phenomenon from a global perspective in order to investigate the resulting near- and far-field acoustics. A RANS simulation was able to achieve similar global level predictions when coupled with the Kirchhoff method by Lee et. al (1994) [36]. Wong et. al (2000) [43] demonstrated that the strongest acoustic signature occurred when the vortex impacted the lower surface of the rotor blade, using a full set of Euler equations in a CFD solver. The understanding of the affect the vortex miss distance $\left(y_{v}\right)$ possesses led to a numerical investigation by $\mathrm{Yu}$ (1996) [48] who used a comprehensive code (CAMRAD-JA) 
coupled with a finite difference code (FPR). It was shown here that changing the blade flapping deformations with a higher harmonic blade pitch control concept can affect the vortex miss distance, and thus the BVI noise can be reduced.

Other areas focusing on the reduction of BVI noise and vibration include work by Gläßel et. al (2001) [34] on the implementation of neural networks into active control mechanisms. This particular study modelled the use of open and closed loop, direct and indirect neural control networks, some of which were capable of learned behaviour using available BVI data. Lee (1994) [47] investigated the potential benefits of including a porous leading edge and found that this treatment was capable of reducing the near field noise by $20-30 \%$.

Research has also been performed which investigated the creation of vortices, either by a starting vortex or tip vortex. Figure 3.9 shows the numerical domain setup used by Mamou et. al (2001) [45] for the study of airfoil-vortex interaction. This study used a shock tube approach to vortex creation, using a heavily downward-pitched upstream airfoil to generate a single coherent vortex structure by exploiting the interaction with an incoming shock wave. Simulations of the tip vortex creation, lifespan and behaviour with age has been covered by Berenger et. al (1997) [5], and found to agree reasonably well with experimental results from within the same study.

\begin{tabular}{|c|c|c|c|}
\hline $\begin{array}{c}\text { Lead Arifoil } \\
\text { (Vortex Generator) }\end{array}$ & Vortex & $\begin{array}{l}\text { S } \\
h \\
o \\
c \\
k \\
W \\
\text { a } \\
v \\
e\end{array}$ & $\begin{array}{l}\text { Stationary Fluid } \\
\text { Aft Arifoil } \\
\text { (Test Airfoil) }\end{array}$ \\
\hline
\end{tabular}

Figure 3.9: Schematic for numerical shock tube used by Mamou et. al (2001) [45] just after the shock wave has past over the vortex-generating airfoil, causing a single, coherent vortex to be shed.

\subsubsection{Experimental Study of Blade (Airfoil)-Vortex Interaction}

Much like its numerical counterpart, the experimental investigation into the blade-vortex interaction has been quite vast. The methods invoked to recreate the vortex in a wind 
tunnel situation vary from moving (pitching $[33,49,50]$, oscillating $[51,52]$ ) to stationary (shock tube [36], blade tip [53]) airfoils located at some distance upstream of the test rotor. The vortex is generated at the forward airfoil, usually a NACA 0012 or NACA 0018 type, and allowed to convect downstream, much like with the numerical treatment. Both subsonic $[33,36,49,51-53]$ and transonic $[50,53]$ cases have been investigated experimentally.

Much of the experimental work that has been done has exploited the two-dimensional nature of the parallel head-on blade-vortex interaction, as it is a much simpler event to measure. Using a dual-pulsed holographic inferometer technique coupled with blade pressure measurements, Lee et. al (1994) [36] were able to demonstrate that viscous effects play a significant role in the head-on BVI case. The method of vortex introduction was a fixed, highly pitched stationary NACA 0018 airfoil interacting with a shockwave to exploit the starting vortex caused by the step function in lift experienced. The holographic inferometer and numerical study results of a head-on BVI is shown in Figure 3.10.

The other vortex creation method utilizing the bound vorticity that is shed as a starting vortex is the rapidly pitching airfoil. Seath et. al (1989) [33] used an upstream impulsively pitching airfoil with a pressure-tapped test rotor to make comprehensive measurements within a subsonic wind tunnel. The effects the maximum pitch angle of the vortex generator has on the resulting vortex were included in this investigation, as well as the effect of vortex position and free stream velocity.

Another study which brought into question the validity of ignoring viscous effects in the numerical study of BVI, was the work by Straus et. al (1990). Using a pitching NACA 0012 airfoil vortex creation method, validated with hot-wire anemometry measurements of the resulting vortex, it was determined that inviscid theory predicts pressure distributions on the target airfoil only in specific situations.

Kalkhoran et. al (1992) [50] conducted two-dimensional transonic wind tunnel tests. Similarly, these measurements utilized the pitching NACA0012 vortex generation method and collected target rotor pressure measurements at various vortex miss-distances.

An oscillating NACA 0012 airfoil was used to create a street of counter-rotating vortical structures in a subsonic wind tunnel by Booth et. al (1990) [51] and (1986) [52] to make 

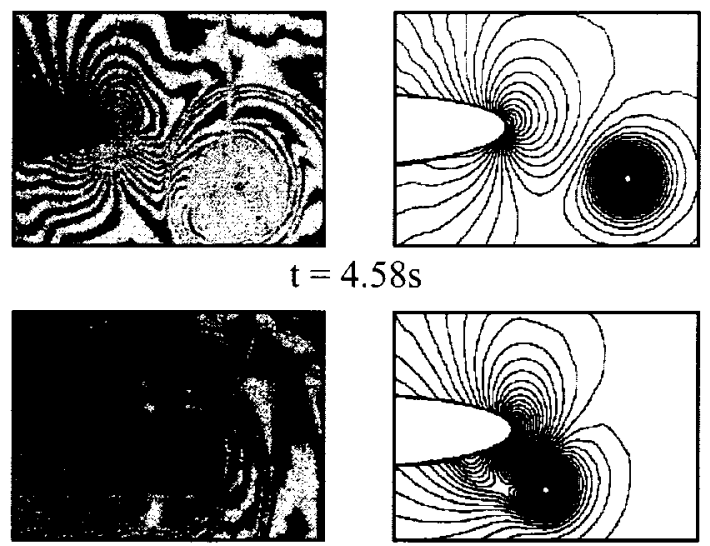

$\mathrm{t}=5.02 \mathrm{~s}$

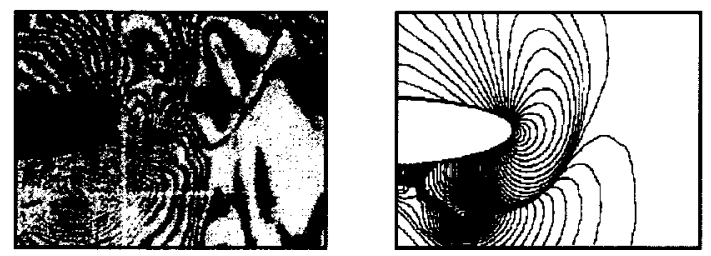

$\mathrm{t}=5.17 \mathrm{~s}$

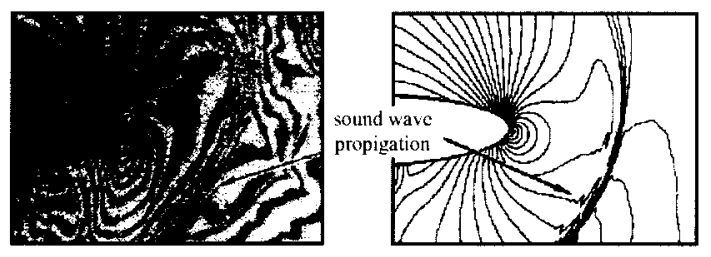

$\mathrm{t}=5.32 \mathrm{~s}$

Figure 3.10: Experimental (left) and numerical (right) results for a head-on collision and the generation of a sound wave [36].

BVI measurements at various miss-distances. A flow visualization technique using smoke, a strobe light and still photography was used, and the authors were able to show that the trajectory of the incoming vortices were influenced heavily at small miss-distances. It was also shown that small miss-distances led to distortion of the vortex core.

One of the few full three-dimensional BVI investigations was done by Caradonna et. al (1988) [53] using the true tip vortex method to introduce the vortex into the flow. A pitched NACA 0015 airfoil was placed perpendicularly upstream of the spinning two-blade test rotor (see Figure 3.11). The tip vortex formed at the end of the vortex generator convects downstream to the test rotor when any number of BVI measurements can be performed, depending on the location of the test rotor set up. A steady vortex sheet having 
a non-dimensional circulation of $\Gamma=0.406$ and core radius of $a_{0}=0.379$ was observed. Pressure measurements with transducers located on the rotating blades were made. Threedimensional measurements were also made by Berenger et. al (1997) [5] of the evolution of the tip vortex in rotorcraft. The creation of the tip vortex and its behaviour after leaving the rotor blade were investigated using laser-doppler anemometry (LDA). A three-dimensional vortex generator was used to study the propagation of a vortex downstream by Doolan et. al (1999) [54]. Hot wire anemometry measurements were used to validate the vortex creation method.

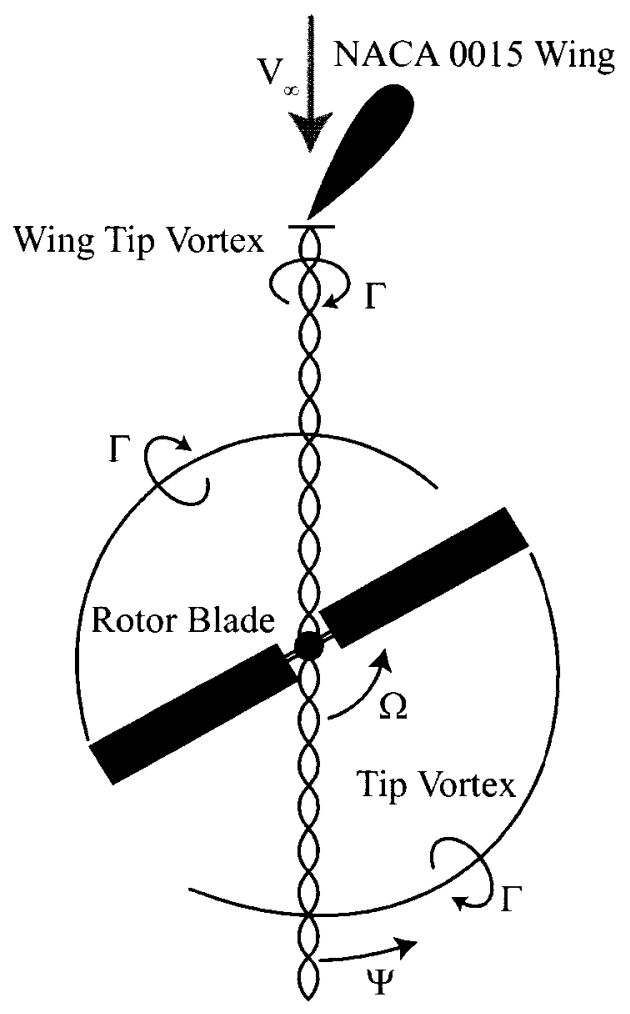

Figure 3.11: Schematic for wind tunnel rotor BVI test by Caradonna et. al (1988) [53].

The accuracy of embedded vortical structures in numerical simulations was investigated experimentally in two-dimensions by Malkiel et. al (1999) [55]. A comparison between numerical modelling of a vortex and experimental actualization of such a flow was made and found to show good agreement. 


\section{Particle Image Velocimetry in Blade (Airfoil)-Vortex Interaction}

Due to the non-intrusive nature of the PIV measurement technique and the relatively high frequency repeated measurements can be made, it has become a popular tool for BVI (or AVI) investigations.

Raffel et. al (2004) [4] completed a fully three-dimensional BVI study utilizing threedimensional PIV. This study was to serve as a proof-of-concept for three-dimensional PIV blade-vortex interaction measurements. Seeding particles created with a Laskin nozzle generator, less than $1 \mu \mathrm{m}$ in diameter and composed of Di-ethyl-hexyl-sebacat (DEHS) were released into the flow upstream of the target test area. During the measurements a significant decrease in seeding density was observed within the vortex core, due to the decreased air density at the vortex core and inertial forces felt by the seeding particles in the swirling flow.

A head-on parallel BVI particle image velocimetry measurement was made by Horner et. al (1996) [56]. Expancel-DE20 micro-balloons, having a mean diameter of $20 \mu \mathrm{m}$ and a specific gravity of 0.06 , were used to seed the flow. The PIV measurements indicated a very strong vortex distortion and when compared with surface pressure BVI measurements, agreed quite well.

A problem with PIV blade-vortex interaction measurements is the ability to capture an image pair of a vortex at the exact (or near) same location for hundreds of measurements for averaging. Green et. al (2000) was able to increase accuracy of the PIV measurements of the BVI event by utilizing two separate PIV systems acquiring measurements in very close temporal proximity to one another. This effectively doubled the number of measurements available, but also increased the chances each time that a measurement was made with the vortex core located at the desired location due to the timing. These measurements were conducted in a low speed wind tunnel and used the rotating vortex generator described in Doolan et. al (1999) [54].

Many types of BVI do not always have flow constrained to two dimensions like the parallel BVI does. This provides a challenge for making PIV measurements when there is a 
very strong out-of-plane fluid motion present. Grant et. al (1994) [57] devised a geometrical method to alleviate this problem and improve PIV measurements where this strong out-ofplane flow is present.

Green et. al (2005) [58] studied the flow around a sinusoidally pitching symmetric airfoil with particle image velocimetry, capturing the ensuing vortical flows brought on by the oscillatory pitching motion. Hollow glass spheres, measuring $11 \mu \mathrm{m}$ in diameter, seeded the water tunnel in which these measurements were made. The final vector field consisted of 100 averaged instantaneous flow measurements.

The trailing tip vortices of wind turbine blades were investigated with PIV by Grant et. al (2000) [59]. Vortex meander was a problem observed in this study as well, and employed the method of selective measurements based on vortex core location used by Devenport et. al (1996) [60], Horner et. al (1996) [56] and Green et. al (2000) [3]. 


\section{Chapter 4}

\section{Experimental Setup}

\subsection{Wind Tunnel and Test Rotor}

In order to recreate the blade-vortex interaction event in the laboratory, a chamber where tests could be performed in flows at known velocities and turbulence levels was required. A two-dimensional wind tunnel was designed and constructed by Brassard [61] specifically for PIV measurements of an airfoil-vortex interaction (AVI) event. The wind tunnel was designed using a closed-loop concept, for the purpose of seeding particle retainment within the wind tunnel. The wind tunnel consists of a blower, wide angle diffuser, settling chamber, set of flow guiding screens, contraction nozzle, test section, curved diffuser and corner vanes, to wrap the circuit back to the blower. The blower selected was the General Purpose Acoustafoil 33 inches centrifugal blower, made by the New York Blower Company. The remaining components were custom made for the specific design of the wind tunnel. The wind tunnel flow speed was controlled via the blower frequency, with settings from $0.5 \mathrm{~Hz}$ to $60 \mathrm{~Hz}$. Figure 4.1 shows side view of the wind tunnel with the components of the tunnel labeled.

The cross-section of the test section of the wind tunnel is $127 \mathrm{~mm}$ deep by $737 \mathrm{~mm}$ tall, and $1524 \mathrm{~mm}$ long in the flow-wise direction. A slot in the centre of the bottom of the wind tunnel, $1067 \mathrm{~mm}$ long and $25 \mathrm{~mm}$ wide, was present to allow for the PIV laser to pass through. A $5 \mathrm{~mm}$ thick sheet of glass was fixed to the underside of the test section, overtop of the slot, such that it would not leak air, but still allow the laser sheet to enter 


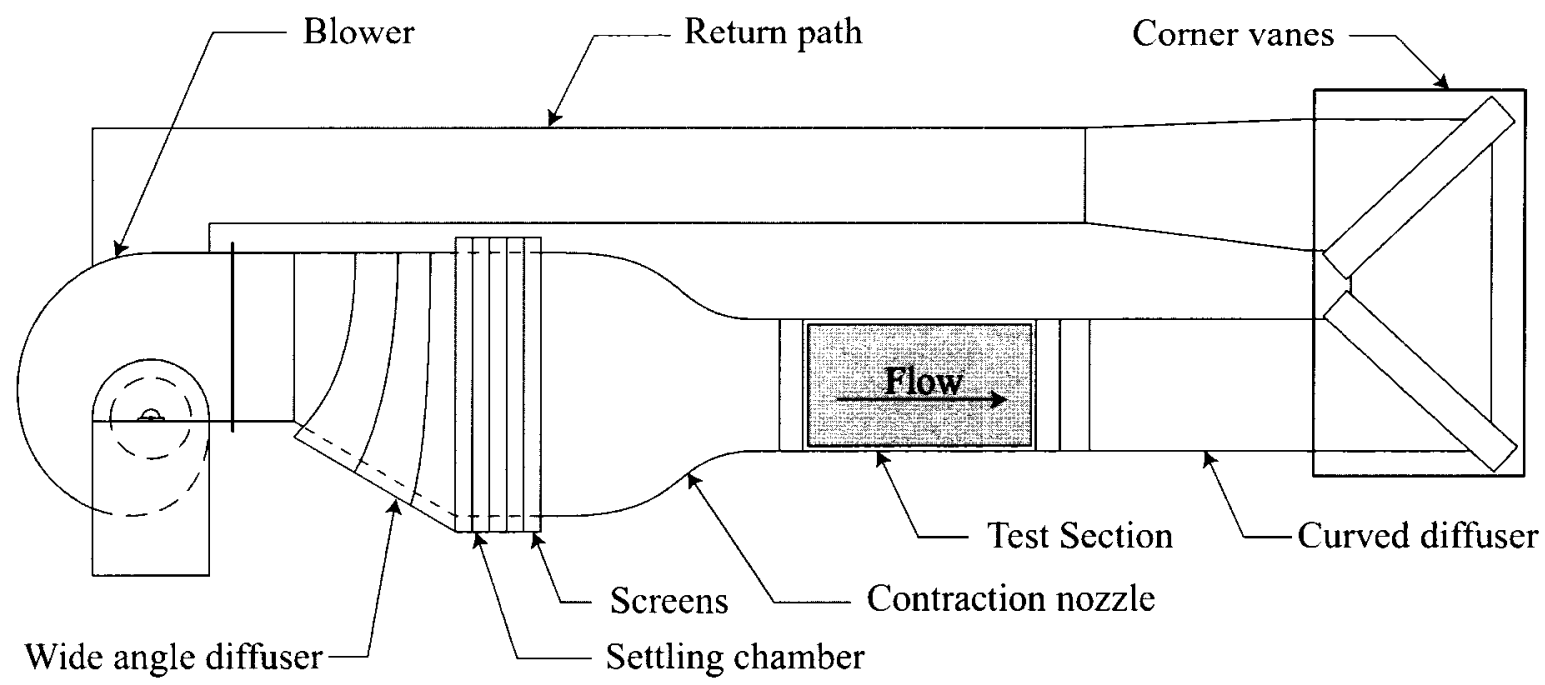

Figure 4.1: Side view schematic of the wind tunnel as designed and constructed by Daniel Brassard [61]. The individual components of the wind tunnel are labeled.

the wind tunnel. The entire (non-Plexiglass portion) inside of the wind tunnel test section was treated with a matte-black spray paint in order to minimize reflection of the powerful PIV laser. Figure 4.2 shows the test section as it was implemented with the installation of both the test rotor and the vortex generating unit as well as the PIV laser.

The range of blower frequency settings represents a near linear range of $0 \mathrm{~m} / \mathrm{s}$ to approximately $90 \mathrm{~m} / \mathrm{s}$ of velocities in the test section at the point where measurements are taken. The velocity profile over the centre-line of the test section at the inlet and outlets, for three different blower settings are shown in Figure 4.3. Angularity tests were conducted within the test section of the wind tunnel by Jeffrey Wilkins as an M.Eng project. The results of these angularity tests are shown in Figure 4.4.

From the centre-line velocity measurements, it can be seen that there is acceptable uniformity of the velocity over the centre-line of the wind tunnel-at both the inlet and outlet. It is assumed that the flow remains uniform through the entire centre-line of the test section, from the inlet to the outlet, where the measurements were taken. A maximum of $0.3 \%$ deviation from the average centre-line flow, not including the two uppermost and lower most data points (due to boundary layer effects), was observed for the inlet, while a maximum of $1 \%$ velocity deviation was observed along the outlet centre-line. 


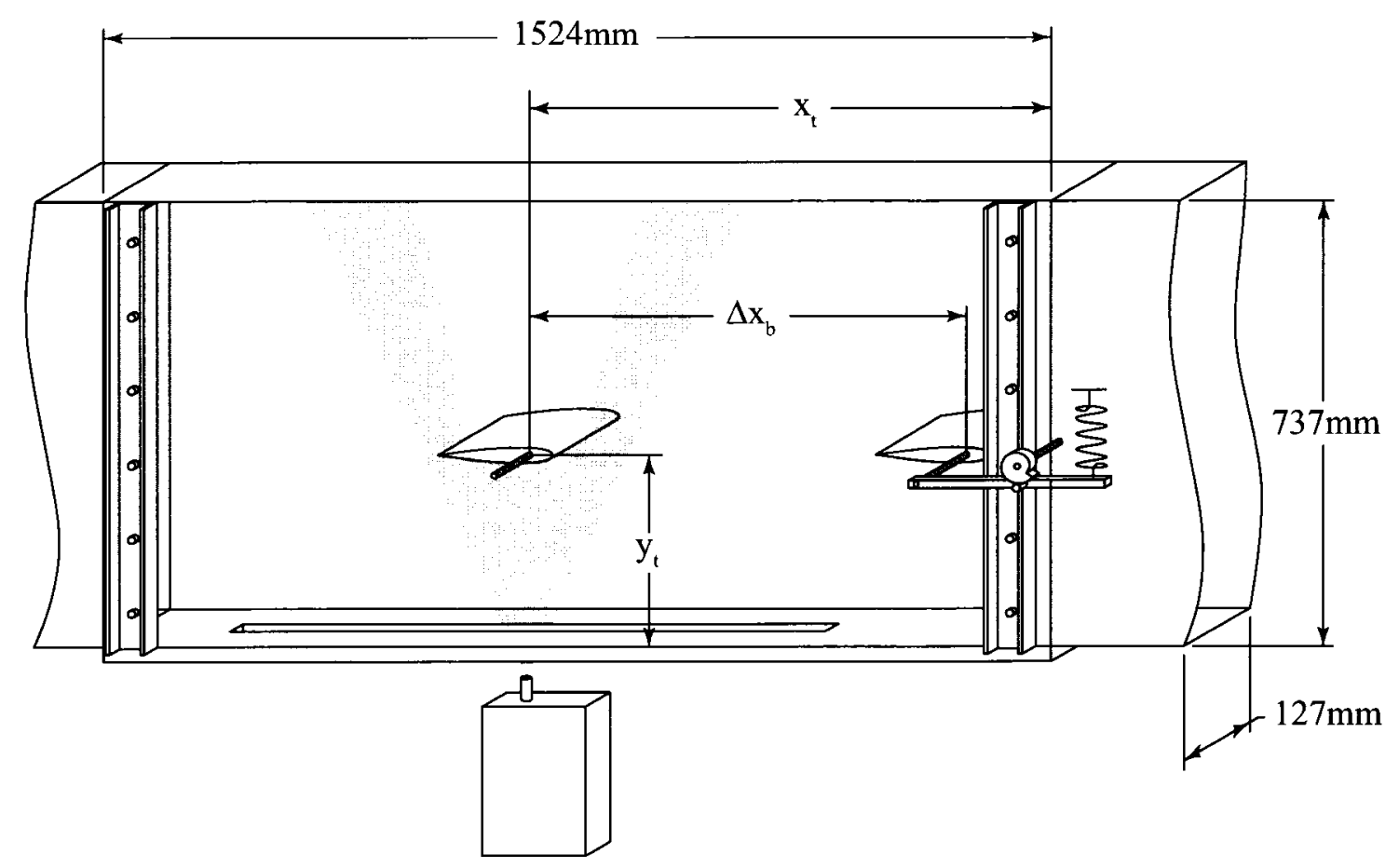

Figure 4.2: Sketch of the wind tunnel test section set-up for blade vortex measurements. The blade separation is held constant at $\Delta x_{b}=2 \bar{c}=400 \mathrm{~mm}$ and the vertical position of the test rotor is altered to change the miss-distance of the incoming vortex.

The angularity data shows that the largest absolute angularity for the $20 \mathrm{~Hz}, 40 \mathrm{~Hz}$ and $60 \mathrm{~Hz}$ fan speed settings were $1.3^{\circ}, 1.5^{\circ}$ and $1.6^{\circ}$, respectively.

The test rotor used was a solid aluminum, symmetric, NACA 0012 airfoil with a chord length of $200 \mathrm{~mm}$ and width of $123 \mathrm{~mm}$, to span the width of the test section. The blade was treated with a matte-black paint since laser reflections were unwanted. The blade was mounted in the wind tunnel on a fixed threaded rod, which passed through the airfoil at the quarter-cord point. This was bolted against the sides of the test section from the exterior of the wind tunnel. The test rotor was mounted $y_{t}$ from the bottom of the test section, which depended on the measurement that was being made, and $x_{t}=550 \mathrm{~mm}$ from the entrance of the test section. For all the measurements conducted during this study, the angle of attack of the test rotor was held at $0^{\circ}$.

In order to vary the miss-distance of the incoming vortex from the test rotor, the test rotor was moved vertically to the appropriate miss-distance from the constant location of 


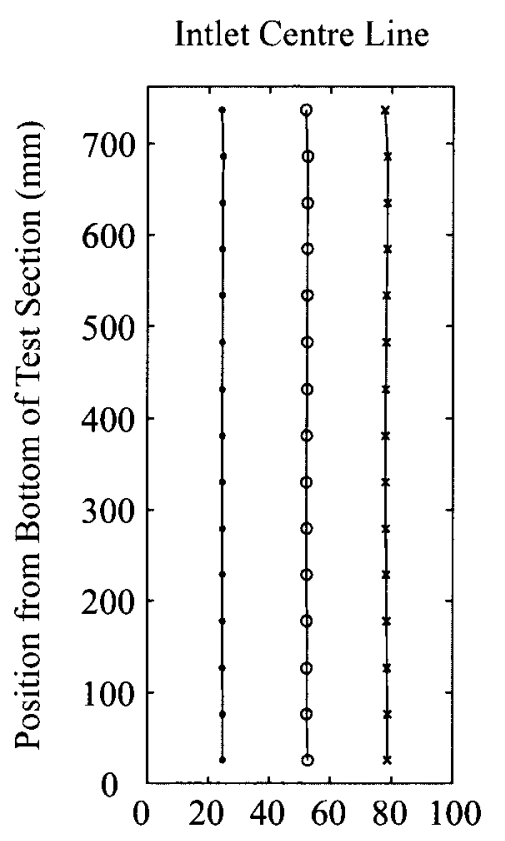

Flow Velocity $(\mathrm{m} / \mathrm{s})$
Outlet Centre Line

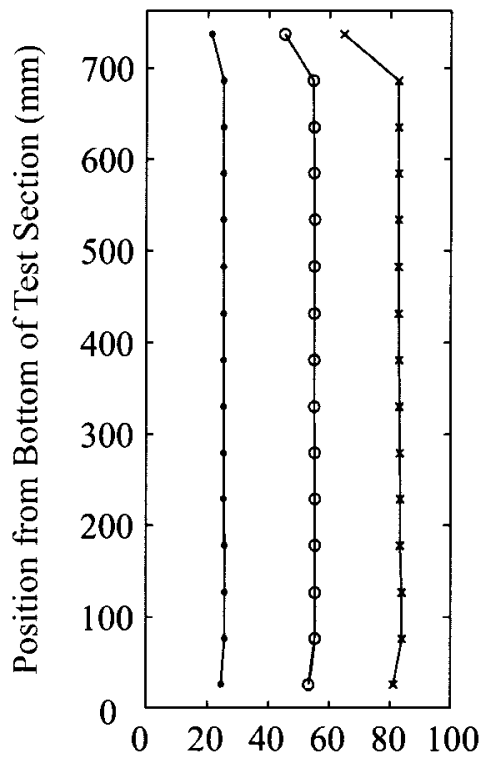

Measurement lines

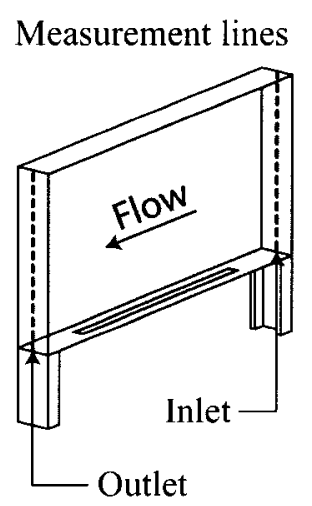

- $20 \mathrm{~Hz}$ Fan Speed

- 40Hz Fan Speed

$\times 60 \mathrm{~Hz}$ Fan Speed

Flow Velocity $(\mathrm{m} / \mathrm{s})$

Figure 4.3: The velocity profiles, for $20 \mathrm{~Hz}, 40 \mathrm{~Hz}$ and $60 \mathrm{~Hz}$, at the inlet (left) and outlet (right) of the test section, measured along the vertical centre-line. These velocity profiles are in the plane which is made by the laser sheet.

the vortex path. This was done purely due to the simplicity of moving the test rotor rather than the vortex generating unit.

\subsection{Vortex Creation}

To observe an AVI event it was necessary to introduce a vortex in the flow, at some distance upstream from the test rotor. This proved to be a non-trivial task involving numerous designs. The creation of the vortex in the wind tunnel was accomplished utilizing the vortex-creating property of the pitching airfoil method described in Section 3.2.

A solid aluminum NACA 0012 (symmetric) blade having a cord length of $200 \mathrm{~mm}$ and span of $123 \mathrm{~mm}$ ( $4 \mathrm{~mm}$ shorter than the width of the test section to allow for slip along the walls), was mounted in the wind tunnel, $150 \mathrm{~mm}$ from the entrance of the test section and $352 \mathrm{~mm}$ from the bottom of the test section. This corresponds to $2 \bar{c}$, or two chord lengths, upstream of the test rotor in the test section. 


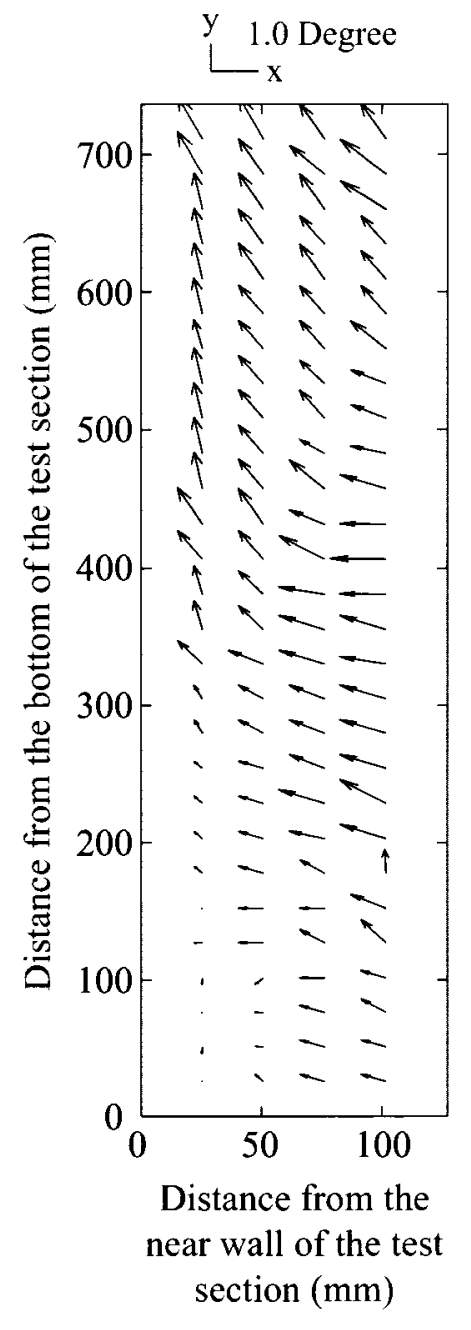

20Hz Fan Speed

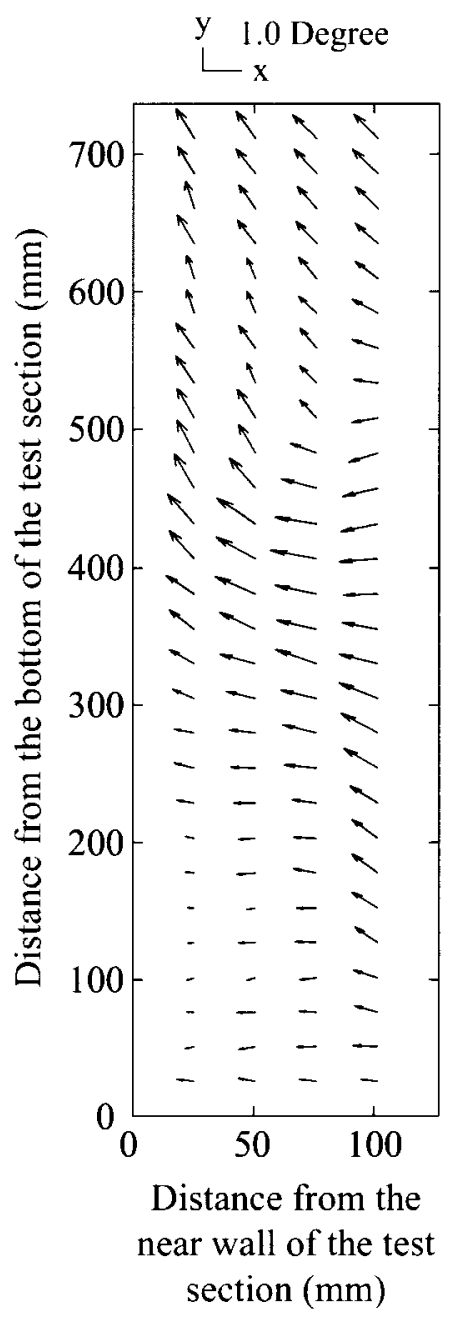

40Hz Fan Speed

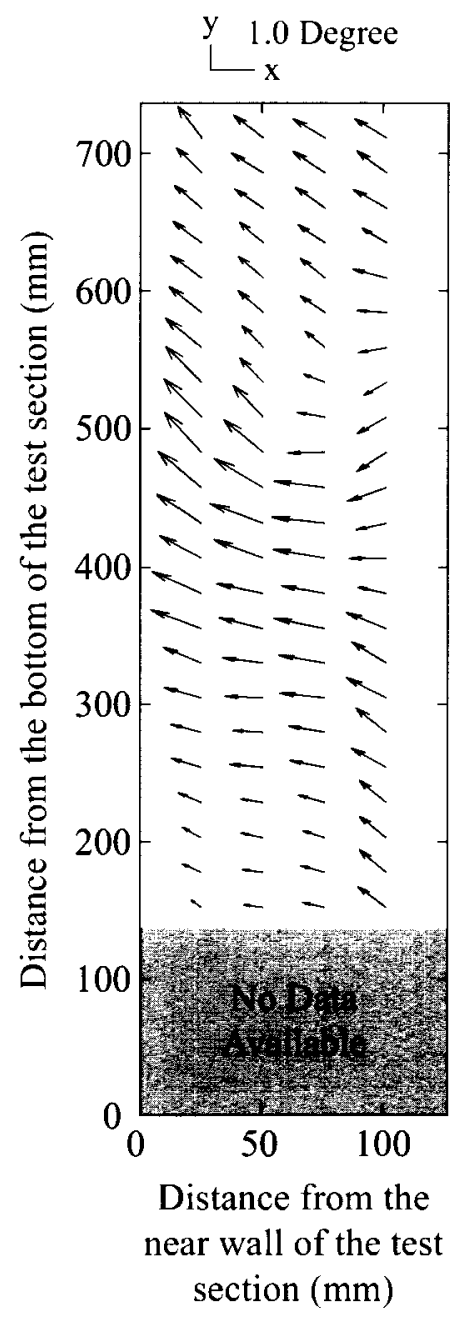

$60 \mathrm{~Hz}$ Fan Speed

Figure 4.4: Angularity measurements, for $20 \mathrm{~Hz}, 40 \mathrm{~Hz}$ and $60 \mathrm{~Hz}$, through the cross section of the test section at the point where the test rotor was mounted.

The vortex generating airfoil was mounted via a threaded rod through the quarter chord point of the blade, or $50 \mathrm{~mm}$ from the leading edge. This threaded rod pass through small holes in the side walls of the test section. Bearings were mounted on the exterior of the side walls, such that the rod, when placed through them, rotated freely of the holes in the side walls. A layer of felt was fastened to the sides of the blade in order to prevent air from passing between the walls and the blade, as well as to protect the walls from being scratched. Figure 4.5 shows the vortex generating blade, as implemented in the wind tunnel, minus 
the device responsible for the blades rotation.

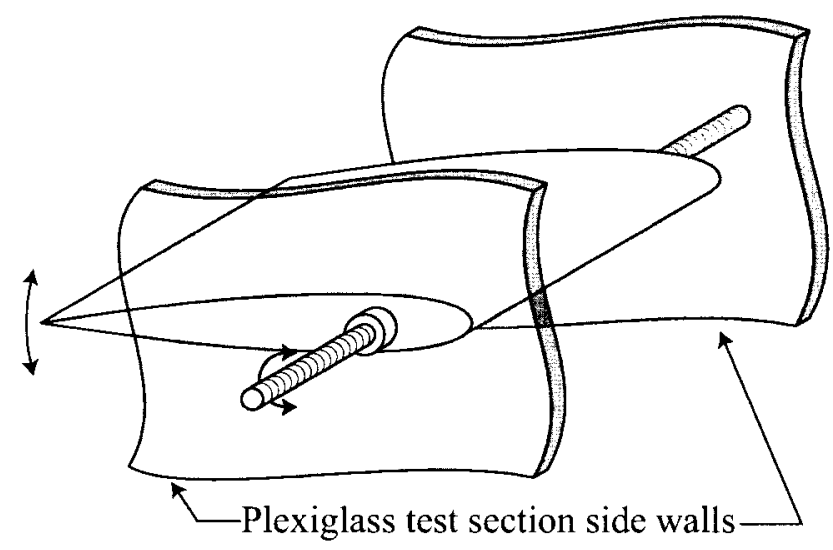

Figure 4.5: The vortex generator blade, showing the mounting method in the wind tunnel.

The blade is rotated via a lever arm attached to the threaded rod. A wheel located at the midpoint of the lever arm rides on the surface of a variable radius cam, where the wheel is forced against the surface of the cam by a spring located at the opposite end of the lever arm. The radius of the cam varies linearly with polar angle from $27 \mathrm{~mm}$ to 41 $\mathrm{mm}$ over the full $360^{\circ}$. Thus, there is a downward step in the radius located at $360^{\circ}$ back to the original radius. As the cam is rotated, the wheel at the midpoint of the lever arm is linearly displaced with respect to the centre of the position-fixed variable radius cam. Since the end of the lever arm which is connected to the blade's threaded rod is fixed in location, the lever arm will rotate about the quarter chord axis of the blade and, being well fastened to the threaded rod, pitch the blade as well.

As the cam is rotated through $360^{\circ}$ and passes over the downward step the force on the opposite end of the lever arm provided by the spring quickly slams the wheel to the surface of the cam with the smaller radius, thus providing the blade with an impulsive pitching motion. The apparatus was arranged such that the blade, as the cam was rotated (via a hand-crank), pitched slowly nose down to $-3^{\circ}$ angle of attack and then near-instantaneously pitched nose up to $+3^{\circ}$ angle of attack as the downward step passed the wheel. This action results in circulation being shed from the tail of the blade given by equation 3.2. Figure 4.6 shows the full vortex generation unit as installed in the wind tunnel.

The angular velocity at which the blade is rotated through the $6^{\circ}$ during the vortex 


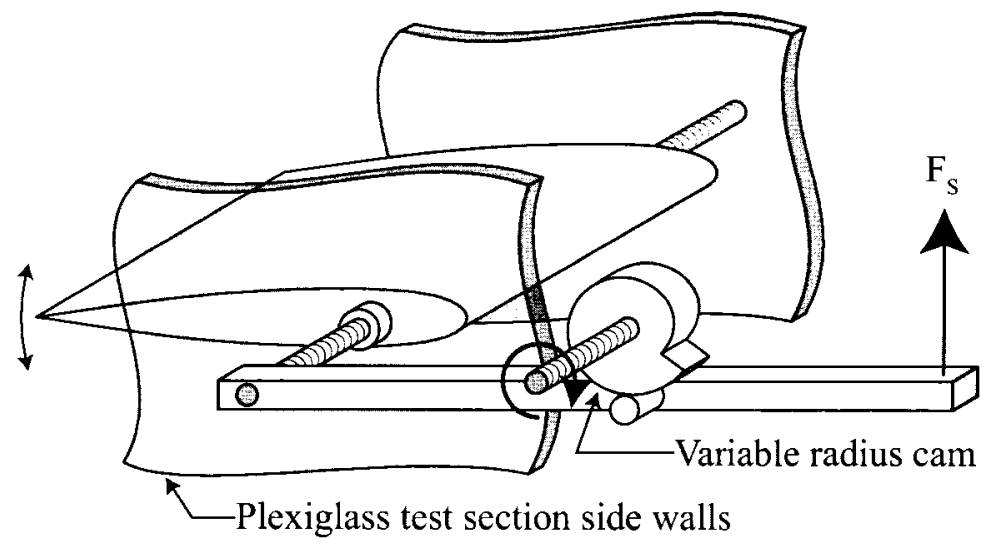

Figure 4.6: The vortex generator as assembled and installed in the wind tunnel

generating motion, when compared to the flow velocity, will have an effect on the shape and behavior of the shed vortex. If the temporal extent of the pitching motion is too large when compared to the spatial movement of the fluid during this time, the resulting circulation, although still conserved, will likely be spread out in the flow and not bundled up as one coherent, relatively discrete vortex that is observed during BVI and ideal for measurements of this type. This leads to an optimization of the pitching motion speed with the related costs and availability of material to handle the necessary impulsive shock that occurs when the blade is stopped suddenly. The speed of the fluid can also be adjusted downward to reduce the necessary compromise, but ideally all flow velocities that the wind tunnel is capable should be available for BVI measurements.

\subsubsection{Timing}

In order to make multiple measurements of subsequent vortices hitting the same location on the airfoil, a necessity for averaging, the PIV system was synchronized with the vortex creation. This was accomplished with a simple electrical circuit (see Figure 4.7) which sent a TTL signal to the external PIV trigger when the vortex generator had reached the vortex creation portion of its cycle, or when the blade reached its $+3^{\circ}$ angle of attack position. The point at which the vortex generating blade triggers the PIV system is somewhat arbitrary, as long as it is repeatable and vortex shedding is consistent. The choice of $+3^{\circ}$ was made 
since the user input to the vortex generation was no longer present at this point and thus would not contribute to error in the timing.

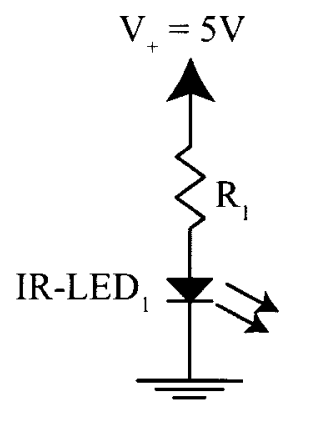

Emitter Circuit

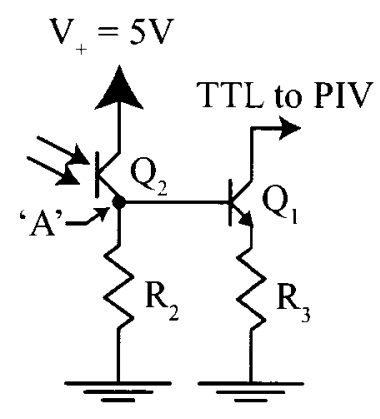

Receiver Circuit

Figure 4.7: The infrared emitter circuit (left) and the infrared detector circuit (right) used to synchronize the PIV system with the vortex creation action.

The emitter circuit is a simple single light emitting diode circuit connected to a regulated $5 \mathrm{~V}$ positive rail, with a resistance of $R_{1}=37 \Omega$ to bias the voltage over the LED to $V_{L E D}$ $=1.3 \mathrm{~V}$ and draw $100 \mathrm{~mA}$ of current. This results in a strong infrared beam from the LED. The receiver circuit uses this infrared beam with an IR phototransistor $\left(Q_{2}\right)$. The phototransistor acts as a switch; when it is receiving the IR beam, it is open and allows current to flow through the resistor $R_{2}=1.42 \times 10^{5} \Omega$. This results in the NPN transistor, $Q_{1}$, opening and shorting the PIV trigger lead. This is the off state of the trigger, with the trigger voltage fluctuation around $0.3 \mathrm{~V}$. This is the collector-emitter voltage in the transistor's on state in addition to the voltage drop over the resistance $R_{3}=39 \Omega$. When the phototransistor no longer is receiving the IR beam, the phototransistor acts as an open switch, or is turned off. This causes $Q_{1}$ to follow suit and as a result, the trigger voltage rises sharply to $5 \mathrm{~V}$, the natural state of the PIV trigger lead. This is the trigger on state.

The implementation of the trigger in to the wind tunnel involved mounting the infrared emitter and receiver facing each other, on the opposite side walls of the wind tunnel test section. They were placed in a manner such that the IR light beam was blocked by the tail of the vortex generating blade, and a trigger signal sent, when the blade's tail reached its lowest position (at $+3^{\circ}$ angle of attack). Figure 4.8 shows the location of the IR beam with respect to the vortex generating blade at neutral angle of attack. Once the IR beam is blocked, 
the voltage at point "A" (see Figure 4.7) falls sharply to $5 \mathrm{~V}$ closing the collector of the transistor, $Q_{1}$, and essentially isolating the positive trigger lead, which quickly becomes its natural state, $5 \mathrm{~V}$. This triggers the PIV system, letting it know that a vortex is incoming to the measurement area. The voltage versus time at the PIV external trigger can be seen in Figure 4.9, which also shows the vortex generator cycle. The PIV software was set up to trigger on the falling edge of the signal, so the lasers fire when the trigger lead is grounded, after an adjustable time delay, and triggering for repeatable vortex measurements is accomplished.

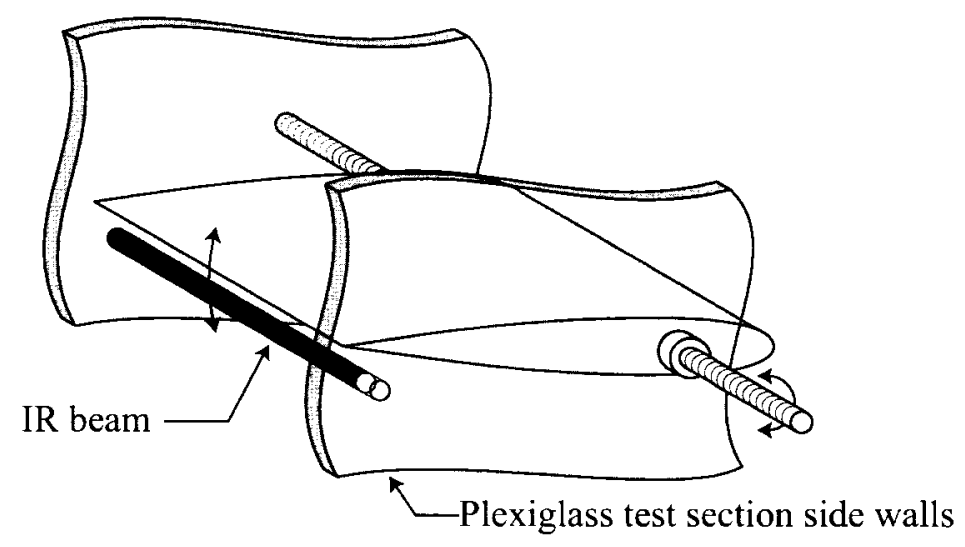

Figure 4.8: The IR beam is broken - and a trigger signal sent - when the tail of the vortex generating blade is at its lowest position, corresponding to an angle of attack of $+3^{\circ}$ for the blade .

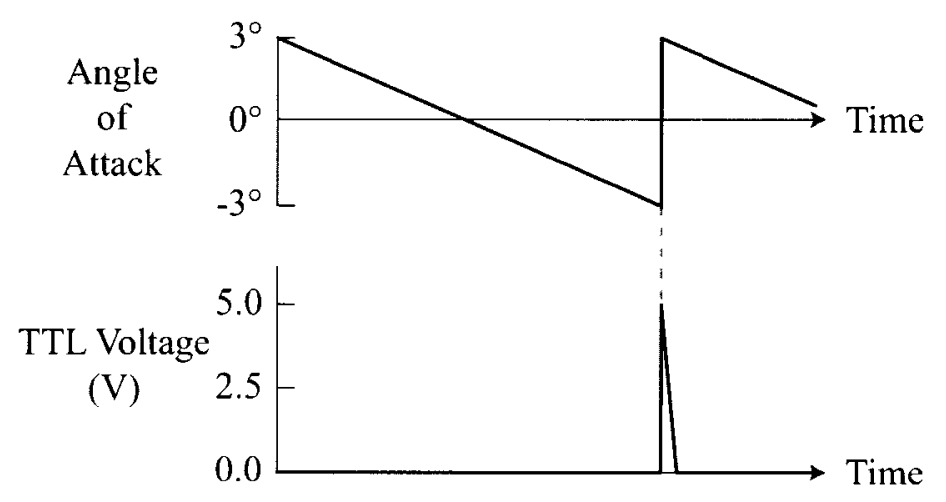

Figure 4.9: Comparison between the TTL signal voltage to the PIV system and the angle of attack of the vortex generating blade through one vortex generation cycle. 


\subsection{Particle Image Velocimetry Apparatus}

The PIV system was assembled and integrated into the wind tunnel at the test section. The laser and camera were both fixed to an anti-vibrational mount. The mount consists of a wooden box filled with $50 \mathrm{~kg}$ of sand resting upon a rubber mat in order to damp out any vibration in the floor from the blower or other vibration sources. A $19 \mathrm{~mm}$ thick aluminum slab with adjustable feet for levelling was fastened to the top of the box with a spring loaded bolt. The camera mount was attached to the slab of aluminum with two vertical stands. The camera mount includes two adjustable rails, allowing for the camera to move parallel $\left(x_{t s}\right.$-direction) with the flow and perpendicularly $\left(y_{t s}\right.$-direction) from the test section side wall. An adjustable jack, mounted on top of the two rails, allows for vertical motion, in the $z_{t s}$-direction, of the camera. The laser is mounted on an anti-vibration breadboard which was fastened to the aluminum slab at a right angle, resulting in the laser being directed upward. This entire assembly rests to the side of the test section such that the camera points towards the test section, perpendicular to the flow, and the laser is directly under the test section with the laser sheet illuminating a sheet parallel to the flow down the centre of the test section (see Figure 4.10).

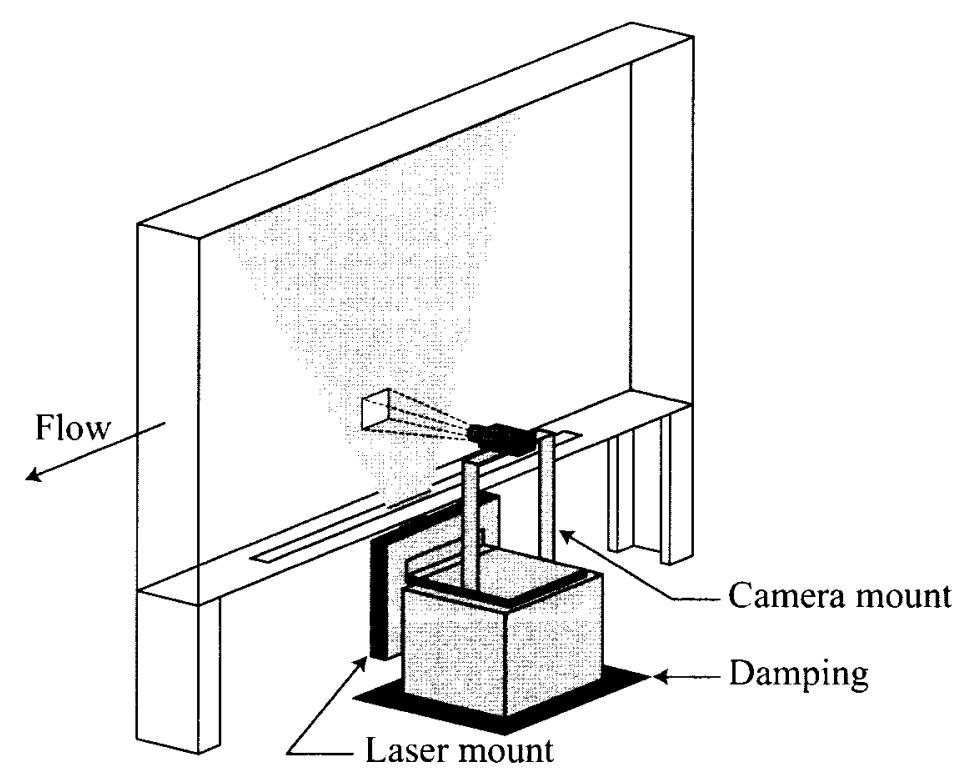

Figure 4.10: Mounting schematic of the laser and camera on the anti-vibrational table at the test section of the wind tunnel. 
The PIV system used was a NewWave Gemini 15 PIV system, consisting of a dual cavity pulsed laser system capable of performing flow measurements at $15 \mathrm{~Hz}$. The lasers used were water cooled, optically pumped Nd:YAG crystal rods with a maximum energy of $120 \mathrm{~mJ}$ per pulse at $\lambda=1064 \mathrm{~nm}$. The laser light was then frequency doubled to $\lambda=532 \mathrm{~nm}$ in a second harmonic generator such that the light was in the visible frequency range.

A 80N35D-FlowMap 1500 processor and related software was used to control and synchronize the PIV system with the camera and external triggers. A 80C50 Kodak Megaplus ES 1.0 camera containing a class 3 CCD chip and equipped with a Nikkon $60 \mathrm{~mm}$ objective lens was used as the imaging portion of the system. The light sheet forming optics (80X62), along with a light-sheet thickness adjuster (80X63), was fastened to the laser unit output to provide the planar illumination in the wind tunnel. The thickness of the light sheet at the measurement area was adjusted to be $1 \mathrm{~mm}$. The laser was set to illuminate at highest intensity possible with both lasers providing the same brightness.

\subsubsection{Tracer Particles}

Seeding of the tracer particles was provided after the test section to allow for a greater distribution of the particles and a more uniform cloud at the entrance to the test section. Also, if any larger, undesired, particles do enter the wind tunnel, they will likely deposit during the first cycle due to inertial effects. The aerosol was created from standard olive oil $\left(\rho=0.92 \mathrm{~g} / \mathrm{cm}^{3}\right)$ using an array of laskin nozzles (ATI, Model TDA-6C, Owings Mills, MD). The olive oil aerosol, after passing through a size-reducing particle impactor, was distributed into the wind tunnel via a streamlined tube, such that the least amount of flow disturbance was introduced, through $5 \mathrm{~mm}$ holes drilled into the tail of the airfoil shape. The streamlined tube used had a chord length of $33 \mathrm{~mm}$ and a width of $13 \mathrm{~mm}$. It spanned the entire $373 \mathrm{~mm}$ height of the diffuser, which is located downstream of the test section, and was installed vertically along the centre-line of the wind tunnel (see Figure 4.11).

The mean particle size that was created by the aerosol generator was approximately $16 \mu \mathrm{m}$ in diameter, which was, unfortunately, rather large for gaseous-based PIV measurements. This was likely due to the absence of an impactor plate within the aerosol generator, 


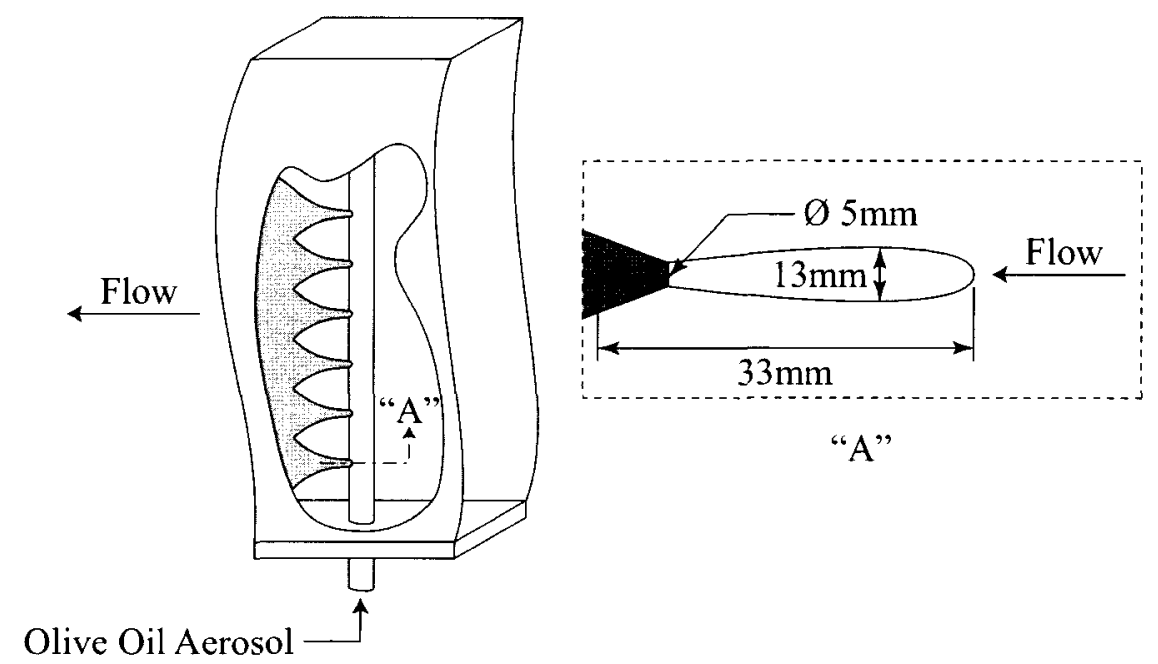

Figure 4.11: Schematic of the seeding rake used in downstream of the test section. Olive oil aerosol that is pumped into the streamlined tube is dispersed through $5 \mathrm{~mm}$ holes.

a component that is standard to most PIV aerosol generators. To circumvent this problem, a particle impactor unit was designed and implemented between the aerosol generator outlet and the wind tunnel inlet. The particle impactor unit consisted of six jet-forming nozzles which were directed downward on to a slightly downwards angled plate (such that deposited olive oil would flow away) at close proximity. The results in the deposition of the larger particle in the olive oil mist and a desired net reduction of the average tracer particle size. After the installation of the particle impactor, size measurements made of particles which reached the exit of the test section (corresponding to at least one complete cycle through the wind tunnel) showed a particle diameter of less than $1 \mu \mathrm{m}$ (see Figure 4.12 for size distribution). The size was determined by sampling the seeded wind tunnel flow using a Particle Size Distribution Analyzer, Model 3603, from TSI Inc. This size of seeding particles is sufficiently small to track the flow for the velocities used in this study.

\subsection{Test Conditions}

The measurements conducted during this study were chosen to demonstrate the capabilities of the PIV system in the wind tunnel at various flow conditions. A convergence analysis was completed, which indicated the number of measurements required to reach a statistically 


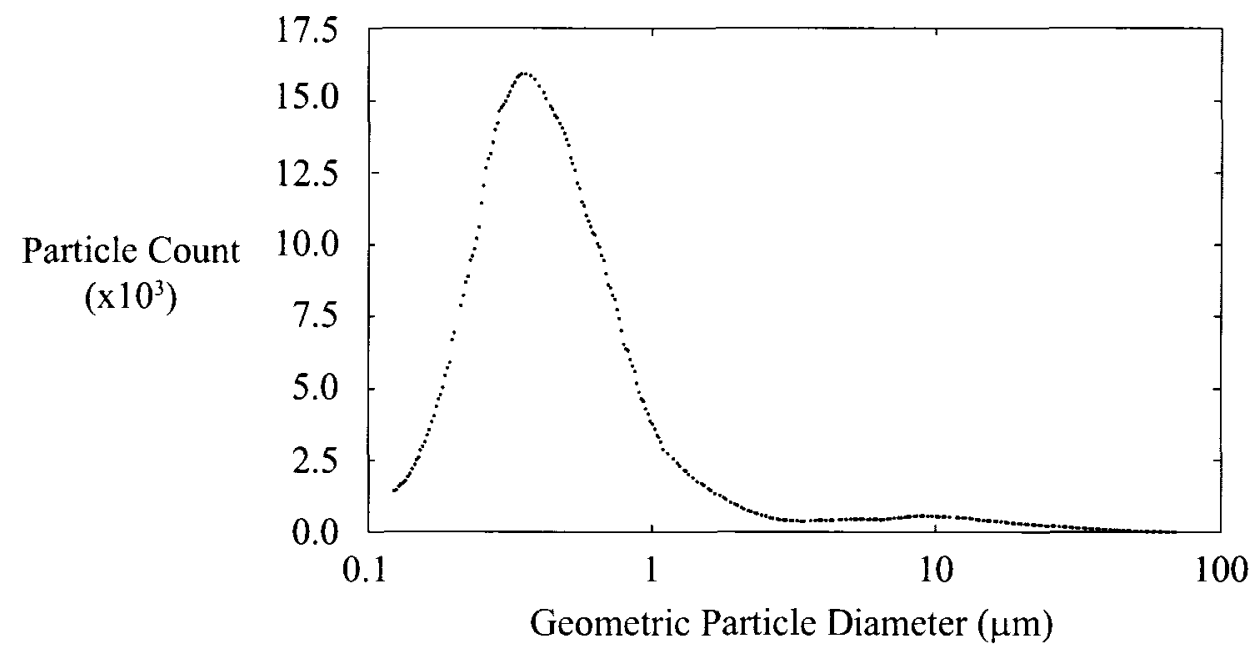

Figure 4.12: Distribution of the olive oil seeding particles diameter. The mean geometric diameter of the particles is $0.495 \mu \mathrm{m}$.

valid average flow. Numerous sensitivity analyses were conducted to refine the different parameters of the experiment, processing and post-processing procedures. The air flow around a NACA 0012 airfoil (test rotor) with no vortex present in the flow was measured, at high and low resolutions, to indicate the PIV systems ability to measure both full field and boundary layer flows. Measurements of the vortex generator wake (held at constant pitch) were conducted, demonstrating the turbulence measuring capabilities of the PIV system. A vortex was introduced into the otherwise undisturbed flow (no test rotor present) and time lapse measurements were taken of its convection downstream. The distance downstream that it was observed was the length of the test rotor, with room for approach and follow through. An airfoil-vortex interaction was also observed, with the same test conditions as above, only with test rotor present at the appropriate position in the path of the vortex.

\subsubsection{Convergence Analysis}

The convergence analysis consisted of taking 10 sets of measurements, each set containing a different number of measurements. The number of data points in each set increased exponentially from 50 to 1000 . The measurements were of the flow about the nose of the NACA 0012 airfoil, a location with a greater-than-average velocity variation, and therefore more likely to cause the most amount of variance in observed velocities from one measurement 
to the next. The array of average velocity vectors of one measurement set is subtracted from the array of another (consisting of a slightly smaller number of measurements) and the difference is the residual. Ideally, the residual should approach zero as the number of measurements used in the average flow field increases. The point at which a greater number of measurements contributing to the average flow field does not reflect a significant change in the residual velocity is taken to be the number of measurements where the flow field is statistically valid.

The convergence analysis yielded an ideal number of measurements of slightly less than 300 , which corresponded to an average residual of $0.05 \%$ for all vectors in the flow field (See Figure 4.13a). Considering only the maximum residual in each vector map, 300 measurements corresponds to a maximum residual of about $0.5 \%, 10$ times the average residual value (See Figure $4.13 \mathrm{~b}$ ).

The residuals of $0.05 \%$ for averaged residuals and $0.5 \%$ for maximum residuals being well below the error expected in PIV measurements, this is sufficient for a statistically valid average. Thus, a set of measurements which was used for averaging included at least 300 measurements such that it was statistically valid.

\subsection{Data Analysis Procedure}

The image pair correlation technique and post processing procedure will be discussed in this section, outlining the parameters used for each case explored. As with the data acquisition, the analysis was accomplished using the FlowManager software, version 3.62 from Dantec Dynamics. All of the data presented underwent the following analysis procedure, regardless of the type of measurement. Further processing was completed depending on the type of measurement that was being analysed.

\subsubsection{Common Analysis Procedures}

For all measurements which included a portion of the test rotor, or were near the edge of the light sheet, the masking technique discussed in Section 2.5 was used to remove these areas 


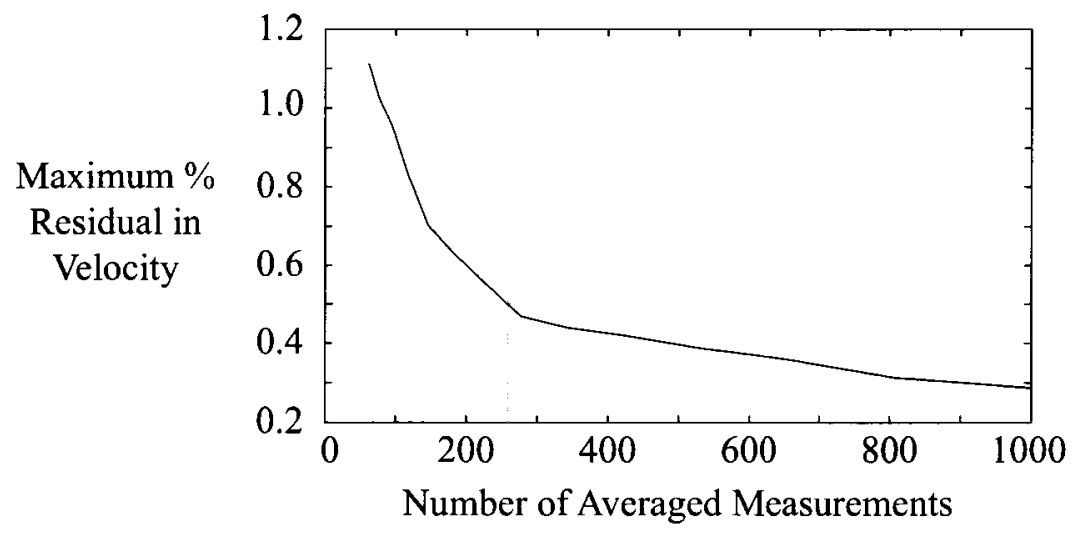

(a)

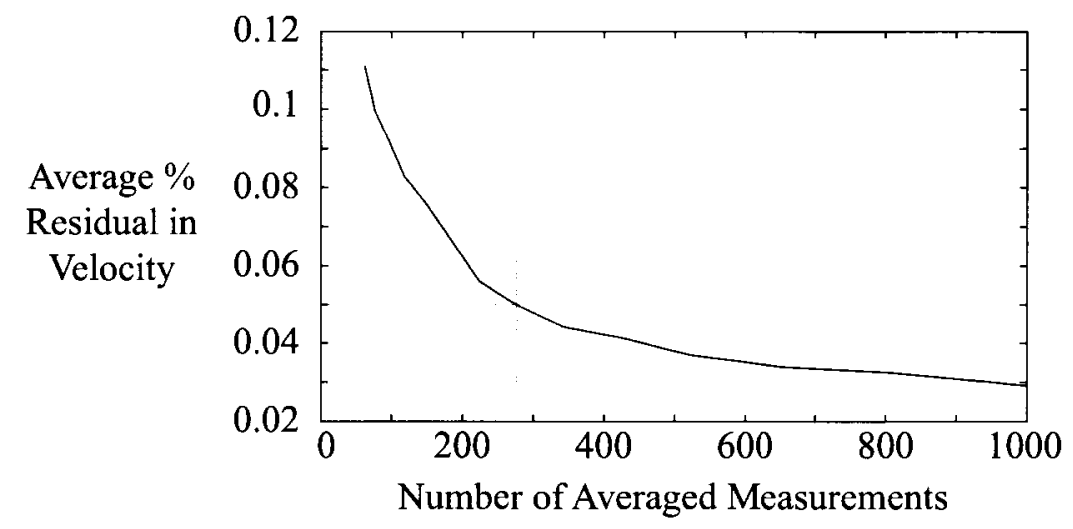

(b)

Figure 4.13: (a) The maximum percent residual in the flow field versus the number of averages used. (b) The average percent residual over the entire flow field versus the number of averages used. On each plot, the grey lines indicate the point at which the percent residual falls below $0.05 \%$ for the average percent residual, corresponding to $0.5 \%$ for the maximum percent residual. Below these residuals (or above the corresponding number of measurements required for such a residual) represents a statistically valid average measurement.

from the correlation process. The mask was applied to the image pairs before correlation and areas which were masked where set to black such that they would not produce any meaningful correlation with any non-masked area.

The masked image pairs were correlated using the recursive local correlation technique outlines in Section 2.6.1, which is referred to in the software by Dantec as the adaptive correlation analysis technique. As was described earlier, this correlation technique involves multiple iterations of cross-correlations, each iteration reducing the interrogation region 
size and using the vector map found in the previous iteration as an offset guide. For the measurements made in this work, a scheme of three iterations resulting in a final interrogation window size of $32 \mathrm{px} \times 32 \mathrm{px}$, from an initial $256 \mathrm{px} \times 256 \mathrm{px}$, was used. The integration window side lengths were halved after each iteration. The vector maps calculated during each iterations are also validated in a three sub-iterations process. The vector maps are validated using the moving average validation technique, as described in Section 2.5. A threshold value of $k=0.06$ was used to validate the vectors. The threshold value was chosen after a sensitivity analysis was performed to optimize the effectiveness of the process.

To increase the vector map resolution and efficient use of image space, as mentioned in Section 2.5, an overlap of $75 \%$ was used in both directions during correlation. Since the FlowManager software employs the FFT method of cross correlation, a top-hat window function and a low-pass gaussian filter function were used to counteract the noise increasing effect, as discussed in Section 2.4.5. The size of the window function used was $k=0.5$ and the threshold value used for the low-pass filter was $k=3.0$. These specific values, like threshold for the validation process of the adaptive correlation process, were chosen through the sensitivity analysis.

The validated, iteratively refined vector maps were then filtered using the average filter described in Section 2.5. The neighbourhood used to calculate the average was a $3 \times 3$ vector area. 


\section{Chapter 5}

\section{Results and Discussion}

The following contour and vector plots describe the flow which was measured in the wind tunnel. The locations of the origins in the plots are arbitrary, and the axes are displayed for scaling purposes only. The testing, processing and post processing parameters are given for each test case. These results indicate the various types of measurements which are available given the current state of the wind tunnel and PIV system.

All of the measurements were done with the same parameters, those which were determined to be optimal through the sensitivity testing. A summary of these parameters are shown below in Table 5.1.

\subsection{Vortex Generator Wake with No Test Rotor Present}

The flow field in the wake of the vortex generating airfoil was measured to determine the amount of flow disturbance which the test rotor will encounter as a result of the presence of the vortex generator upstream. Due to the slight turbulent nature of the flow in the wake of the blade, the capability of the PIV system to capture turbulence information can also be investigated. This can be compared to numerical simulations of the same flow conditions, or hot wire anemometry measurements in the same wind tunnel, and conclusions drawn on the turbulence measuring abilities of the PIV system. Parameters such as scaling factor of the measurements (field of view), tracer particle size and the $\Delta t$, to name a few, can be altered and optimized for turbulence measurements once highly accurate data from a HWA 
Table 5.1: Parameters used in the measurement sets for this study. See Section 2.4 for a complete description of these parameters.

\begin{tabular}{ll}
\hline Parameter & Value \\
\hline \hline Final interrogation window size & $32 \mathrm{px} \times 32 \mathrm{px}$ \\
Adaptive iterations & 3 \\
Refinement iterations & 3 \\
$\Delta t$ & $\frac{N}{4 V_{\infty}}$ \\
Interrogation region overlap & $75 \% \times 75 \%$ \\
Window filter & $k=0.5$ \\
Low-pass filter & $k=3.0$ \\
Moving Average validation area & $3 \times 3$ \\
Average filter area & $3 \times 3$ \\
Data sets averaged & $300(50$ for vortex measurements)
\end{tabular}

system are available for comparison.

The turbulence is calculated from the entire set of 300 flow field measurements of the wake. The average flow (average of the 300 velocity measurements) is subtracted from each individual velocity field measurement. This results in the fluctuation components in velocity throughout the flow field. Each velocity component in the field is then squared, to get the turbulence kinetic energy. This is shown in Equation 5.1:

$$
k_{e}=0.5\left(\left(u^{\prime}\right)^{2}+\left(v^{\prime}\right)^{2}\right)
$$

This gives the turbulence kinetic energy, per unit mass, in the two dimensions of the plane which contains the measurement. The out of plane component $\left(w^{\prime}\right)$ cannot be measured by the current PIV system.

A set of five measurement positions were collected, for fan settings of $20 \mathrm{~Hz}, 40 \mathrm{~Hz}$ and $60 \mathrm{~Hz}$. The first camera position was located $2 \bar{c}$ downstream from the tail of the vortex-generating airfoil. The scale factor for this set of measurements was 11.023, which corresponds to a field of view of $100.0 \mathrm{~mm}$ wide $\times 100.8 \mathrm{~mm}$ tall. An overlap of $25 \mathrm{~mm}$ 
on each seam was allowed to ensure there were no gaps in the final data. This gives a final field of view of $425 \mathrm{~mm}$ wide and $100 \mathrm{~mm}$ tall.

Figure 5.1 illustrates the turbulence levels recorded with the PIV system in the wake of the vortex generator at fan settings of $20 \mathrm{~Hz}, 40 \mathrm{~Hz}$ and $60 \mathrm{~Hz}$. These results strictly provide only a lower bound estimating of the turbulence level present in the wind tunnel and are heavily dependent on the tracer particles' tendency to follow the flow. The actual turbulence motion would be out of phase (leading) and greater than the reported values. Nevertheless, these results do showcase the PIV system's capabilities to measure turbulence, however accurate it may be. When hot wire anemometry measurements are completed, comparisons can be made to give an indication of how accurate or inaccurate this system's turbulence measuring capabilities are.

\subsection{Flow Field for the NACA 0012 Airfoil with no Vortex Impaction}

The flow field in the test section with the NACA 0012 test rotor in place was investigated with no vortex creation or vortex generator present in the wind tunnel. This is the simple case of the flow around a NACA 0012 airfoil. The test rotor was located at $x_{t}=550 \mathrm{~mm}$ and $y_{t}=352 \mathrm{~mm}$ and at $0^{\circ}$ angle of attack. Since the flow on the upper portion of the blade should, in theory, be identical to the lower surface (since the blade is symmetric), the upper surface can be generated from the flow on the lower surface. For the Figures presented here, only the lower portion of the flow field around the airfoil is shown, due to this symmetry. Measurements were made for flow speeds corresponding to $20 \mathrm{~Hz}, 40 \mathrm{~Hz}$ and $60 \mathrm{~Hz}$ fan speeds.

A far field measurement was made and consisted of four different lateral camera positions along the blade. The scaling factor for this set of measurements was 9.549, corresponding to a field of view of $86.6 \mathrm{~mm}$ wide $\times 87.3 \mathrm{~mm}$ tall. The camera was moved along the rail to the four different measurement positions, moving $78 \mathrm{~mm}$ each time to allow for some overlap of the fields of view. 


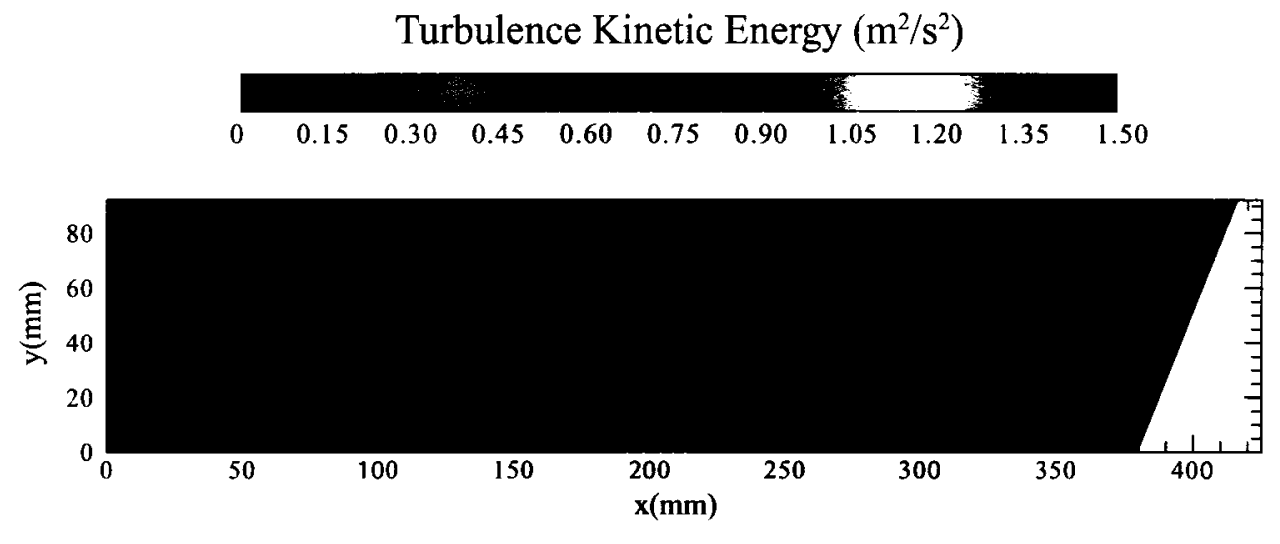

$20 \mathrm{~Hz}$ Fan Speed

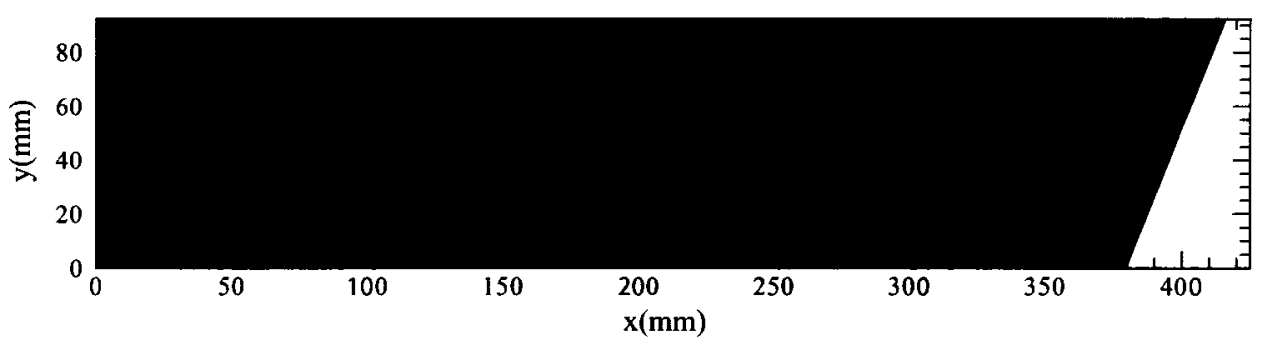

$40 \mathrm{~Hz}$ Fan Speed

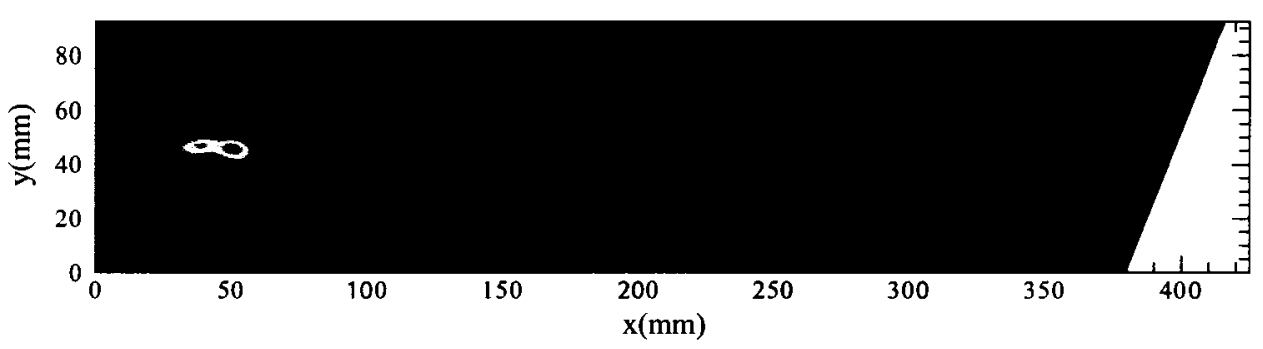

$60 \mathrm{~Hz}$ Fan Speed

Figure 5.1: The turbulence level in the wake of the vortex generator as measured using the PIV system at a $20 \mathrm{~Hz}, 40 \mathrm{~Hz}$ and $60 \mathrm{~Hz}$ fan speeds, corresponding to a free stream velocity of about $24 \mathrm{~m} / \mathrm{s}, 55 \mathrm{~m} / \mathrm{s}$ and $90 \mathrm{~m} / \mathrm{s}$. Flow direction is from right to left, position $450 \mathrm{~mm}$ corresponds to $2 \bar{c}$ downstream of the tail of the vortex generating airfoil.

The velocity contour plots represent the absolute, scalar velocities present at each position in the flow field. The scalar representation is calculated using Equation 5.2:

$$
V=\sqrt{V_{x}^{2}+V_{y}^{2}}
$$


Figures 5.2, 5.3 and 5.4 show the coarse, or wide angle, velocities measurements for the fan speeds of $20 \mathrm{~Hz}, 40 \mathrm{~Hz}$ and $60 \mathrm{~Hz}$, respectively. For these cases, there is no other disturbance in the wind tunnel, and represent the simple case of the air flow over a symmetric, NACA 0012 airfoil (shown as grey in the figure).

Velocity $(\mathrm{m} / \mathrm{s})$
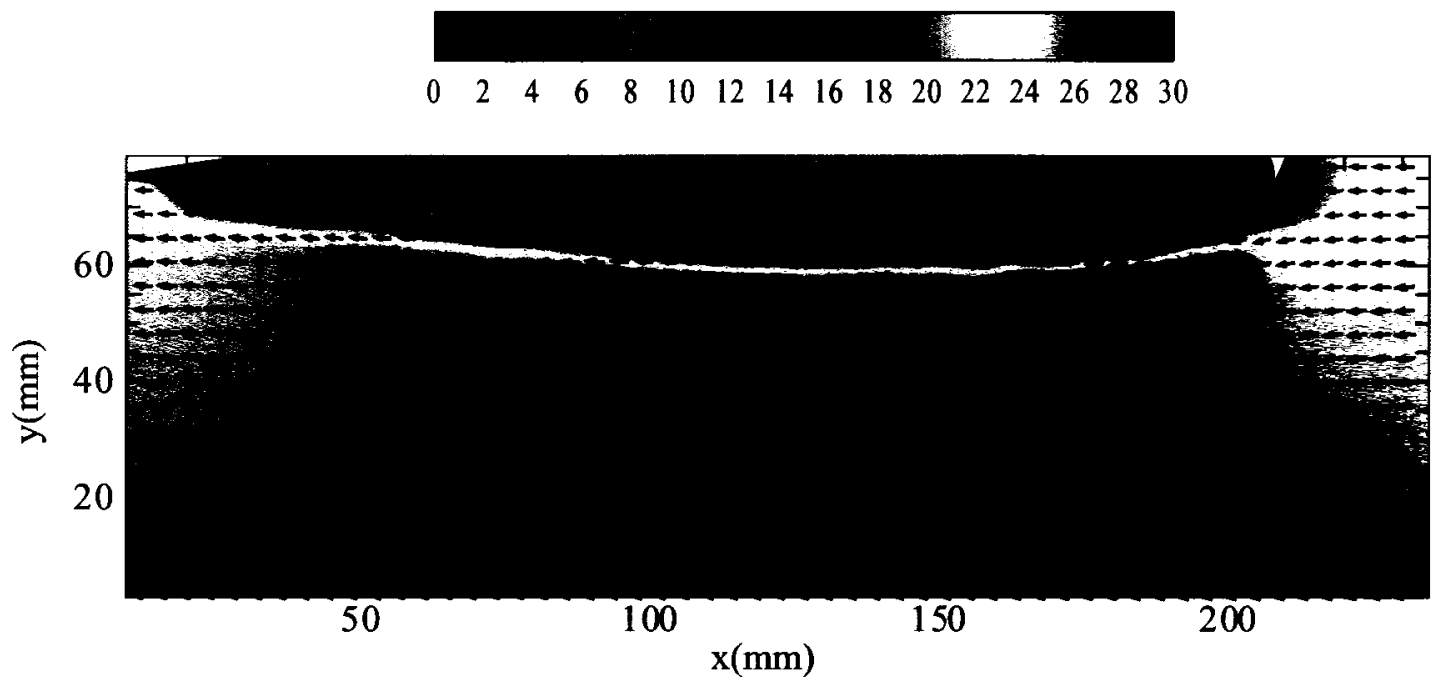

Figure 5.2: The coarse velocity flow field around the NACA 0012 airfoil with no other disturbances in the flow. The fan speed is set to $20 \mathrm{~Hz}$, corresponding to a free stream velocity of about $24 \mathrm{~m} / \mathrm{s}$.

A near field measurement was also conducted, giving finer vector resolution of the flow near the surface of the airfoil under the same conditions. This measurement required thirteen separate lateral camera positions which follow the surface of the airfoil. The scaling factor for this set of measurements was 2.075 , corresponding to a field of view of $18.8 \mathrm{~mm}$ wide $\times 19.0 \mathrm{~mm}$ tall. In this case, the camera was moved along the rail $17.5 \mathrm{~mm}$ for each new camera position, to allow for some overlap in each successive measurement. Figures $5.5,5.6$ and 5.7 show the fine, or close angle velocities for the fan speeds of $20 \mathrm{~Hz}, 40 \mathrm{~Hz}$ and $60 \mathrm{~Hz}$, respectively. The white areas in the results indicate either an area where the shadow of the test rotor obstructed the measurement, or where no measurement was made. Due to the limited field of view at this resolution, only the flow near the surface of the blade was measured, resulting in a disjointed measurement at the seams. The benefit to the high resolution measurements is a much finer measurement and insight into the boundary layer 


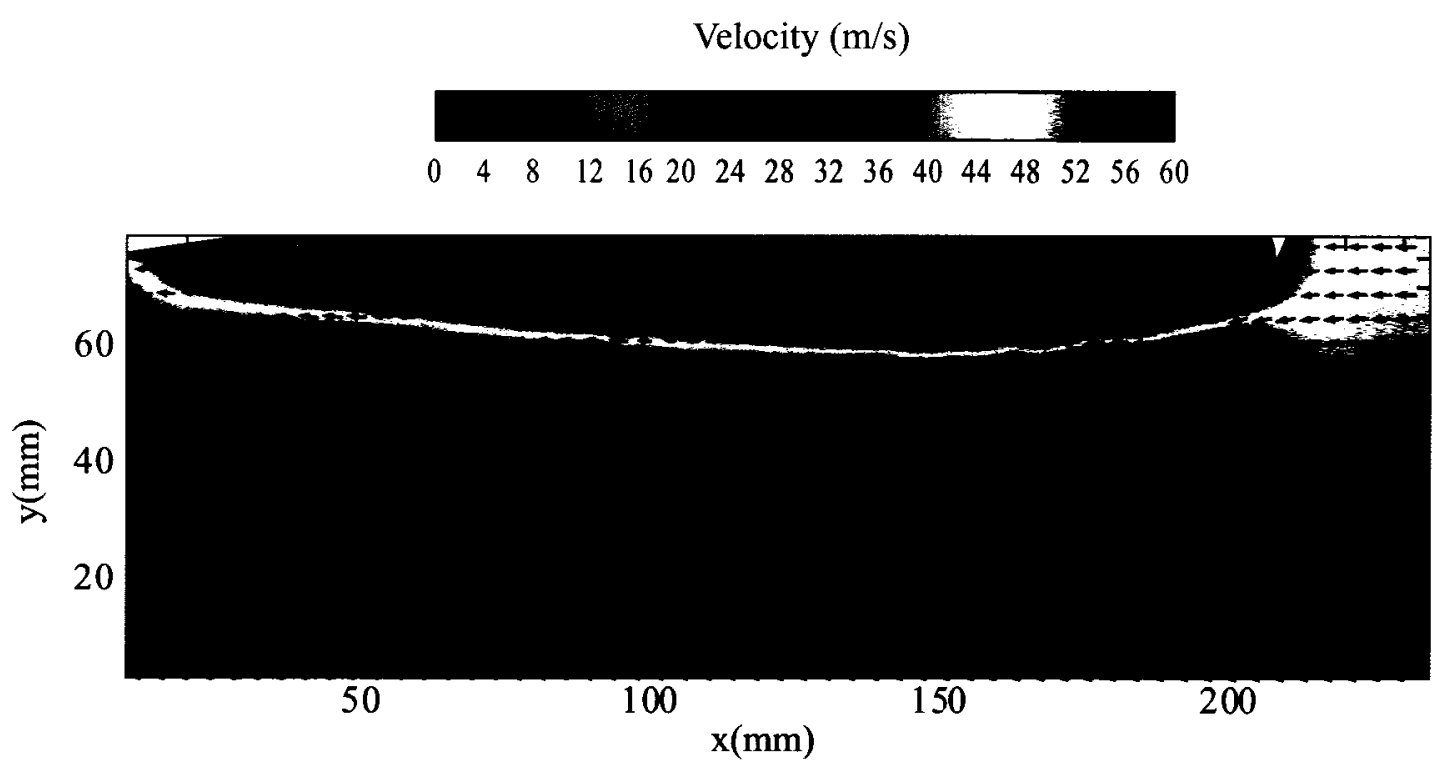

Figure 5.3: The coarse velocity flow field around the NACA 0012 airfoil with no other disturbances in the flow. The fan speed is set to $40 \mathrm{~Hz}$, corresponding to a free stream velocity of about $55 \mathrm{~m} / \mathrm{s}$.

of the airfoil flow. Since the field of view at this scaling factor is so small, however, many camera positions must be used to achieve the entire flow about the airfoil.

The stagnation point at the leading edge of the airfoil can clearly be seen in both measurement sets, and at all three free stream velocities. The region of increased velocity that is expected around any airfoil is also seen at approximately the quarter-chord point of the airfoil. At zero angle of attack, no separation is observed, but the wake downstream of the airfoil can be seen.

\subsection{Flow Field of a Free Vortex}

Measurements were completed of a vortex as it passed through the location where the test rotor was to be mounted. This represents the case where the airfoil-vortex miss distance is infinite. This case can also be used for the characterization of the vortex that the vortex generator provides. The measurements were made by appending four lateral camera positions together, corresponding to the four camera positions required to encompass the length test rotor if it were present. 


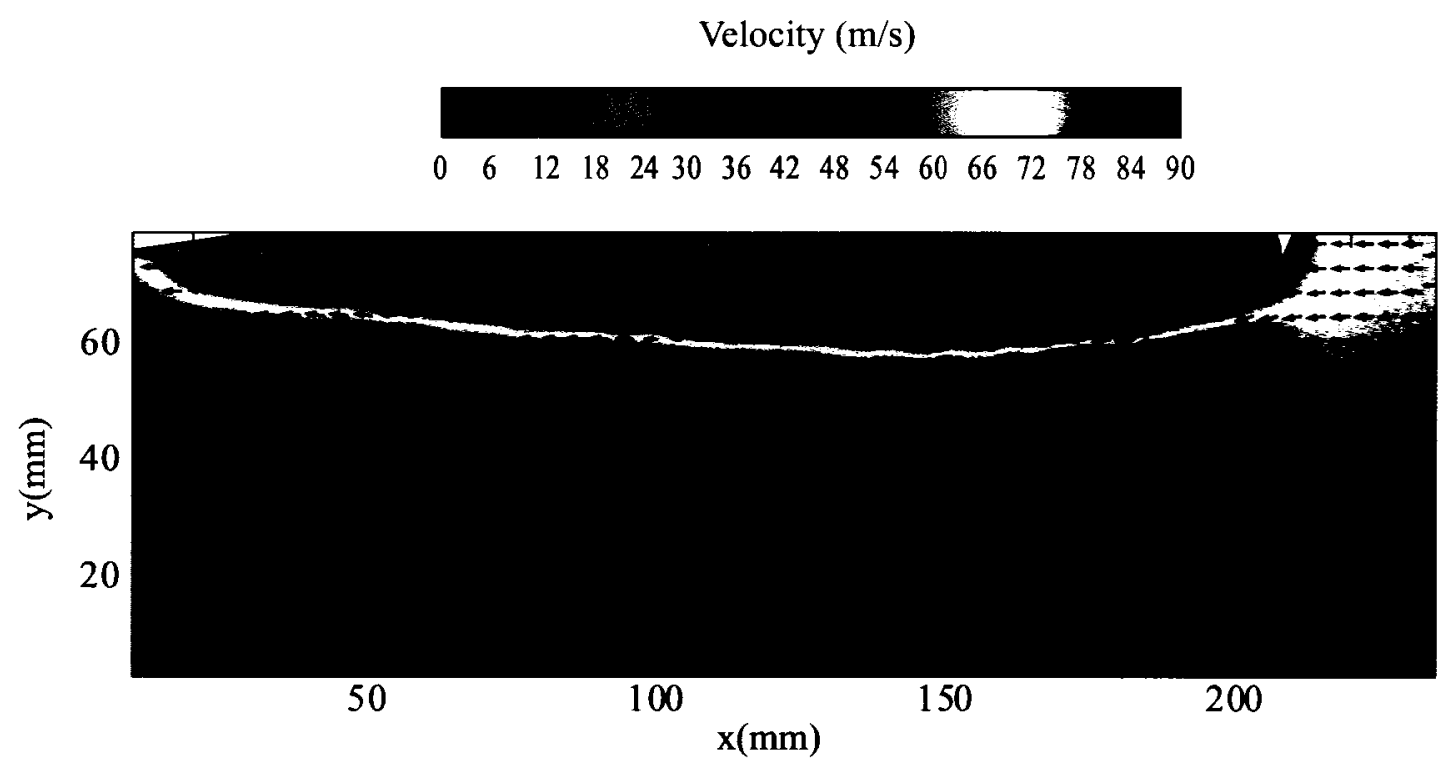

Figure 5.4: The coarse velocity flow field around the NACA 0012 airfoil with no other disturbances in the flow. The fan speed is set to $60 \mathrm{~Hz}$, corresponding to a free stream velocity of about $90 \mathrm{~m} / \mathrm{s}$.

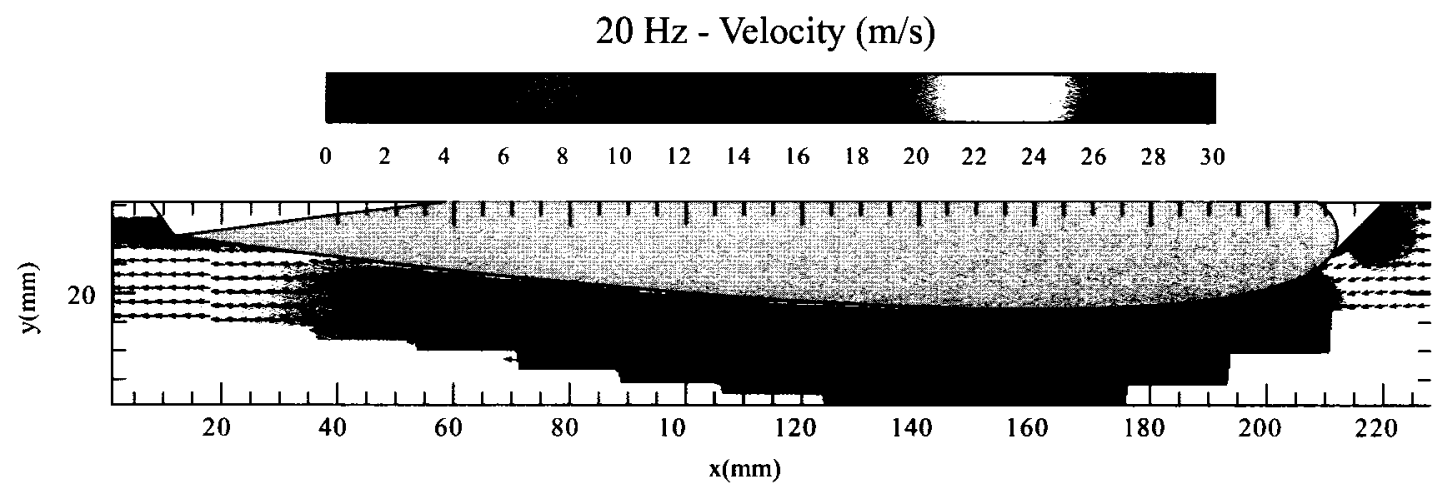

Figure 5.5: The fine velocity flow field around the NACA 0012 airfoil with no other disturbances in the flow. The fan speed is set to $20 \mathrm{~Hz}$, corresponding to a free stream velocity of about $24 \mathrm{~m} / \mathrm{s}$.

The delay between vortex creation and measurement was varied from $18 \mathrm{~ms}$ to $40 \mathrm{~ms}$ in $2 \mathrm{~ms}$ intervals, resulting in 12 frames of an animation of the passing vortex. For each delay, 50 measurements were made and averaged together. The scale factor for this set of measurements was 9.256 , giving a field of view of $84 \mathrm{px}$ wide $\times 84.6 \mathrm{px}$ tall. Figures 5.8 , 5.9 and 5.10 shows the sequence of vorticity measurements of the free vortex from $18 \mathrm{~ms}$ (time zero in the figure) to $44 \mathrm{~ms}$ after after release, which is the length of time it took to 


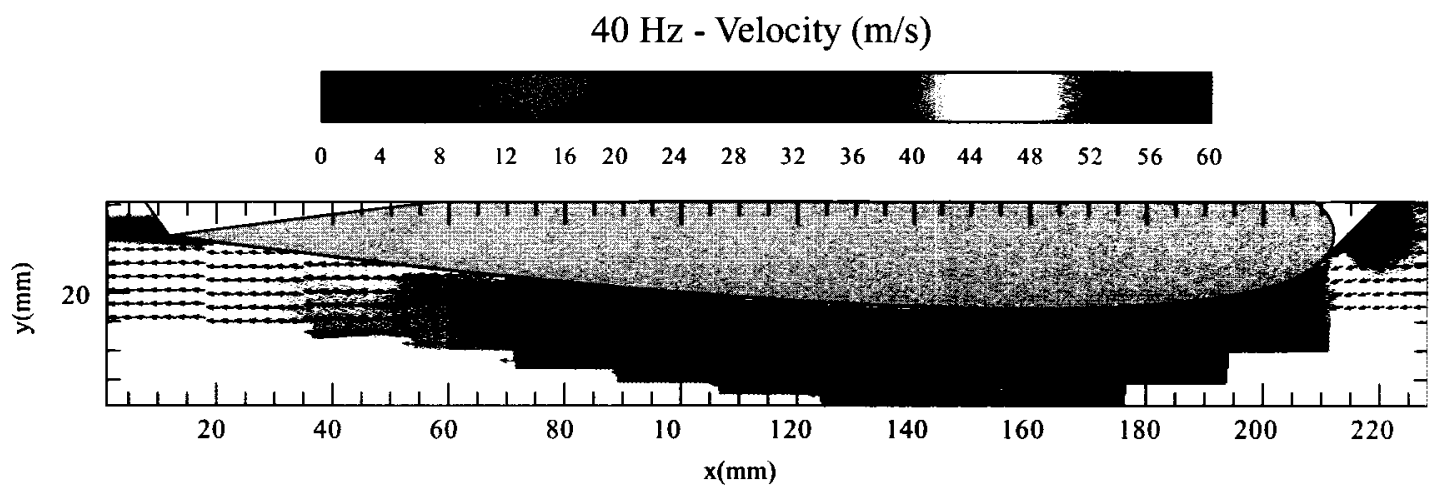

Figure 5.6: The fine velocity flow field around the NACA 0012 airfoil with no other disturbances in the flow. The fan speed is set to $40 \mathrm{~Hz}$, corresponding to a free stream velocity of about $55 \mathrm{~m} / \mathrm{s}$.

$60 \mathrm{~Hz}-$ Velocity $(\mathrm{m} / \mathrm{s})$

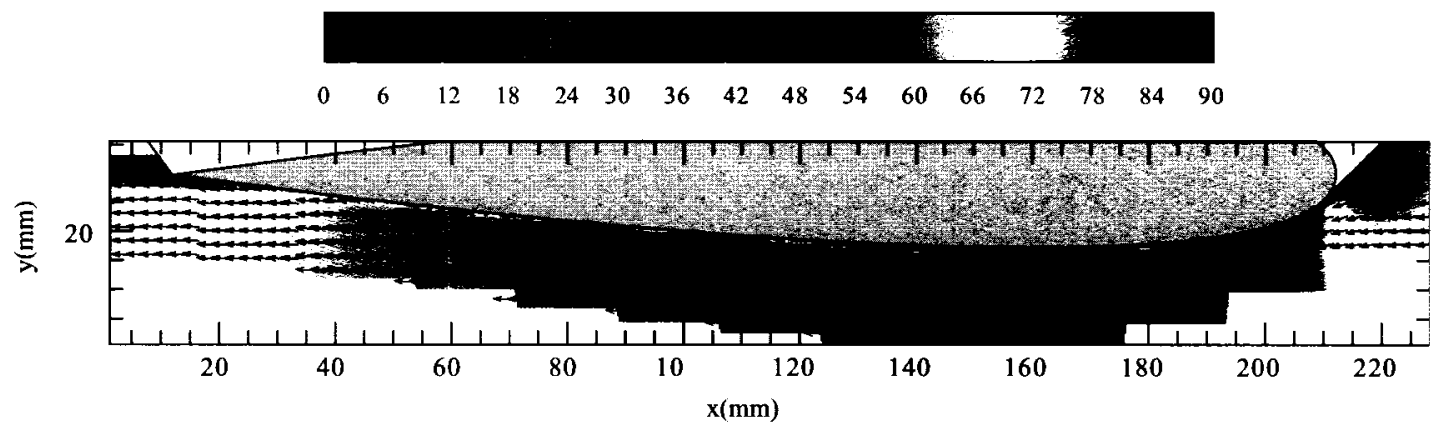

Figure 5.7: The fine velocity flow field around the NACA 0012 airfoil with no other disturbances in the flow. The fan speed is set to $60 \mathrm{~Hz}$, corresponding to a free stream velocity of about $90 \mathrm{~m} / \mathrm{s}$.

pass through the field of view of the measurement.

The vorticity was calculated using the two-dimensional curl of the velocity vector field, through Equation 5.3:

$$
\vec{\omega}=\frac{\partial V_{x}}{\partial x}-\frac{\partial V_{y}}{\partial y}
$$

where $\vec{\omega}$ is in the out of plane direction.

The velocity vectors are also shown to indicate flow direction and relative magnitudes of the average subtracted flow field. Thus, the average flow field at these conditions and the vortex generator held constant at its final position were made and the subsequently 
subtracted from the velocity fields of the individual vortex measurements to enable the visualization of the vortex in the flow with vectors. Figure 5.11 shows the absolute velocity of the flow field as the vortex passes through. This figure did not have the average flow subtracted from it, so the real velocities can be viewed.

The vortex enters the field of view from the right side and convects downstream towards the left. The time at which the measurement was taken after the vortex first entered the field of view is located beneath each frame of the sequence, which is read top to bottom, first collum then the second. An elongated clockwise area of circulation is observed downstream of the singular, counter-clockwise vortex. This can be attributed to the circulation generated and shed as the vortex generating airfoil pitched nose up. Since the pitching motion was not fast enough, the bound circulation would be shed over the full extent of the rotation, the first portion being carried downstream before the last portion was released by the airfoil. This elongated area of circulation was not used to observe a vortex, but the relatively strong and singular counter-clockwise vortex that followed was used. The mechanism for this vortex can be assumed to be the strong-and short-recoil of the vortex generating airfoil after it is suddenly stopped at its $3^{\circ}$ angle of attack final position. The vortex path is slightly downward, an indication that there is some amount of downwash present in the flow due to the final position of the vortex generating rotor. The vortex looses a substantial amount of circulation over the $26 \mathrm{~ms}$ that it was observed. From the first frame to the last frame, the maximum vorticity present at the core of the vortex fell by $50 \%$. There is also a secondary vortex present in the flow, trailing the first by $0.85 \bar{c}$, also counter-clockwise in rotation

\subsection{Flow Field for the NACA 0012 Airfoil with Vortex Im- paction}

The airfoil-vortex interaction measurement conditions were the same as above (for the free vortex), but with the test rotor was mounted in place such that a miss distance of $0.05 \bar{c}=$ $10 \mathrm{~mm}$ was observed. To achieve this, the test rotor was mounted at $x_{t}=515 \mathrm{~mm}$ and $y_{t}=$ $345 \mathrm{~mm}\left(y_{b}=2 \bar{c}=400 \mathrm{~mm}\right)$. Again, four camera positions were used to visualize the entire 
test rotor, since the field of view was $84 \mathrm{px}$ wide $\times 84.6 \mathrm{px}$ tall. The same array of time delays which were used in the free vortex case were also used for the airfoil-vortex interaction measurement. The grey areas in the figures represents the blade, where measurements are meaningless, and the white part are locations where the light sheet could not reach (in the shadow of the test rotor or at either edge of the light sheet).

Figure 5.16 shows the highest resolution of vectors which was available for this measurement, with a frame of the vortex impacting the underside of the test rotor at a miss distance of $0.1 \bar{c}$. This corresponds to the frame of Figure 5.12 at $6 \mathrm{~ms}$ after the vortex entered the field of view.

\subsection{Discussion}

The greatest flow speed at which a single, reproducible, coherent vortex could be produced by the current vortex generation unit, was the $10 \mathrm{~Hz}$ fan speed setting corresponding to a free stream velocity of $11 \mathrm{~m} / \mathrm{s}$. The free vortex measurements and airfoil-vortex measurements were conducted at this flow speed. Since the airfoil free stream flow at $10 \mathrm{~Hz}$ is very similar to that at $20 \mathrm{~Hz}$, the $10 \mathrm{~Hz}$ case was not completed for a simple NACA 0012 airfoil.

The pitching motion that the vortex generating airfoil executed produced clockwise circulation in the flow, as from theory. This was observed quite easily at the higher flow speeds, where a clockwise direction vortex was spread out into a string of small incoherent vortex elements. There was, however, and single, relatively strong, counter-rotating vortex which followed this street, at the $10 \mathrm{~Hz}$ fan setting. This vortex was repeatable and suitable for BVI testing. The source of this vortex is likely the recoil of the blade after it is suddenly stopped at its $3^{\circ}$ angle of attack position. Since the recoil is sharp and short, it does not succumb to the fate of the original vortex and is emitted as a single strong vortex.

This problem can be solved through the re-design of the current vortex generator by increasing the rate at which the airfoil pitches, and future designs of a similar vortex generator should include this. Alternatively, as was put forth by Daniel Brassard [61], a plunging type vortex generator could be developed. This design was developed for for the current 
study, but was found to not work well due to the extreme disruption that it introduced into the flow. It is the authors opinion that a much smaller vortex-generating airfoil would likely work with this mechanism, but could potentially still introduce a significant amount of flow disturbance due to the rather forceful nature of the design.

It was observed that the downwash due to the final position of the vortex-generating airfoil has an effect on the overall vortex path, and should be noted. An adjustment to the final angle of attack for the vortex generating airfoil could solve this issue, but may introduce an up-wash prior to the vortex creation. A smaller angle through which the blade pitches is also a potential solution to this problem. The vortex did not retain its vorticity long and dissipated to less than half its initial intensity by the time it traveled the chord of the test rotor. 

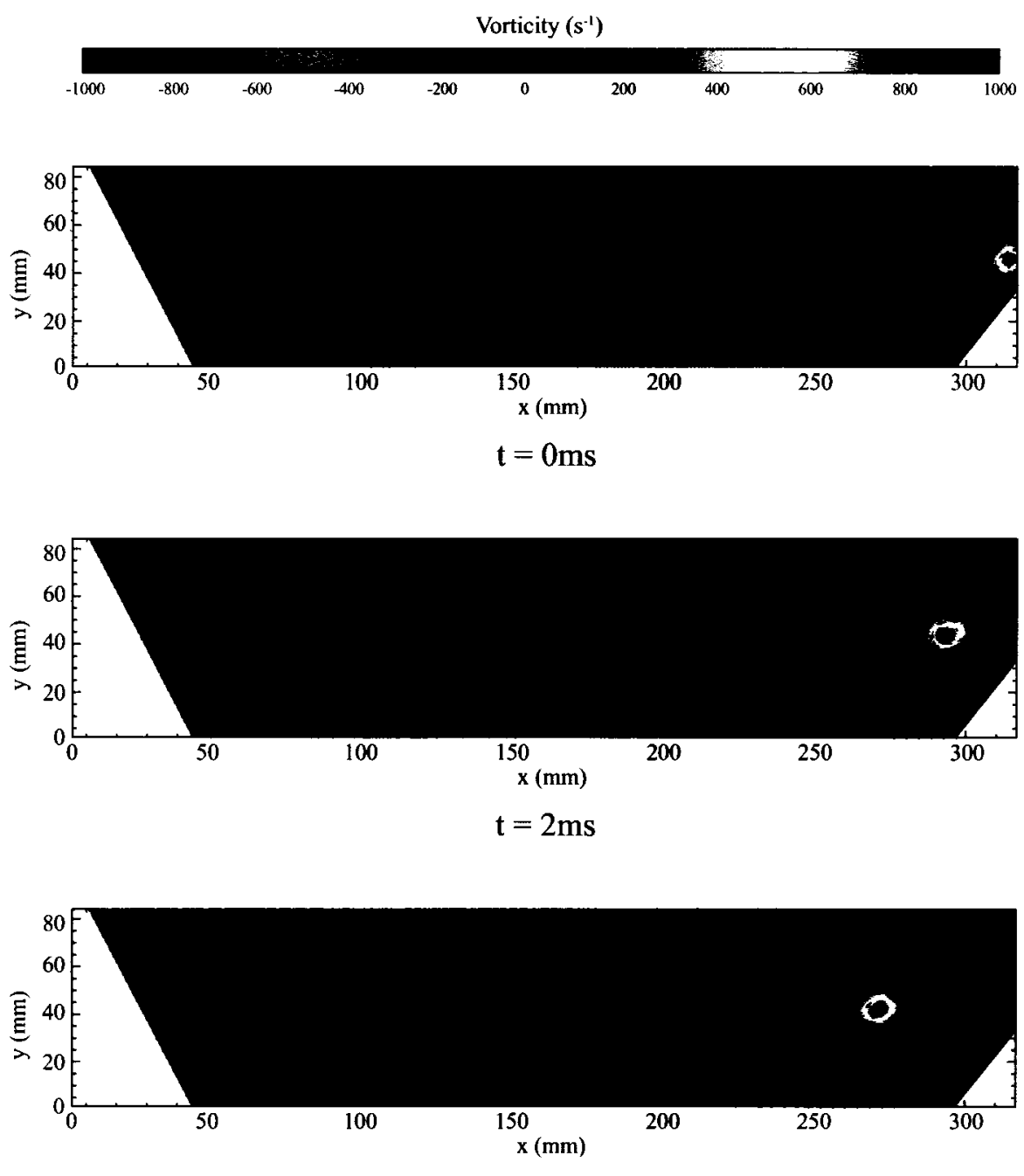

$\mathrm{t}=4 \mathrm{~ms}$

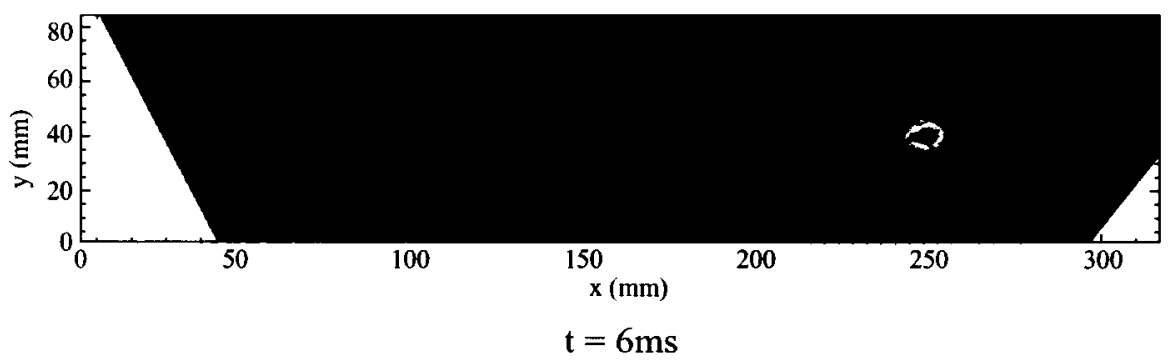

Figure 5.8: The vorticity in the flow for the free-vortex case, $0 \mathrm{~ms}$ through $6 \mathrm{~ms}$; equivalent to an approximate vortex miss-distance of $\infty$, with average-subtracted velocity vectors to demonstrate flow direction and relative magnitude. Measurement at a fan speed of $10 \mathrm{~Hz}$, which corresponds to a free-stream velocity of about $11 \mathrm{~m} / \mathrm{s}$. 

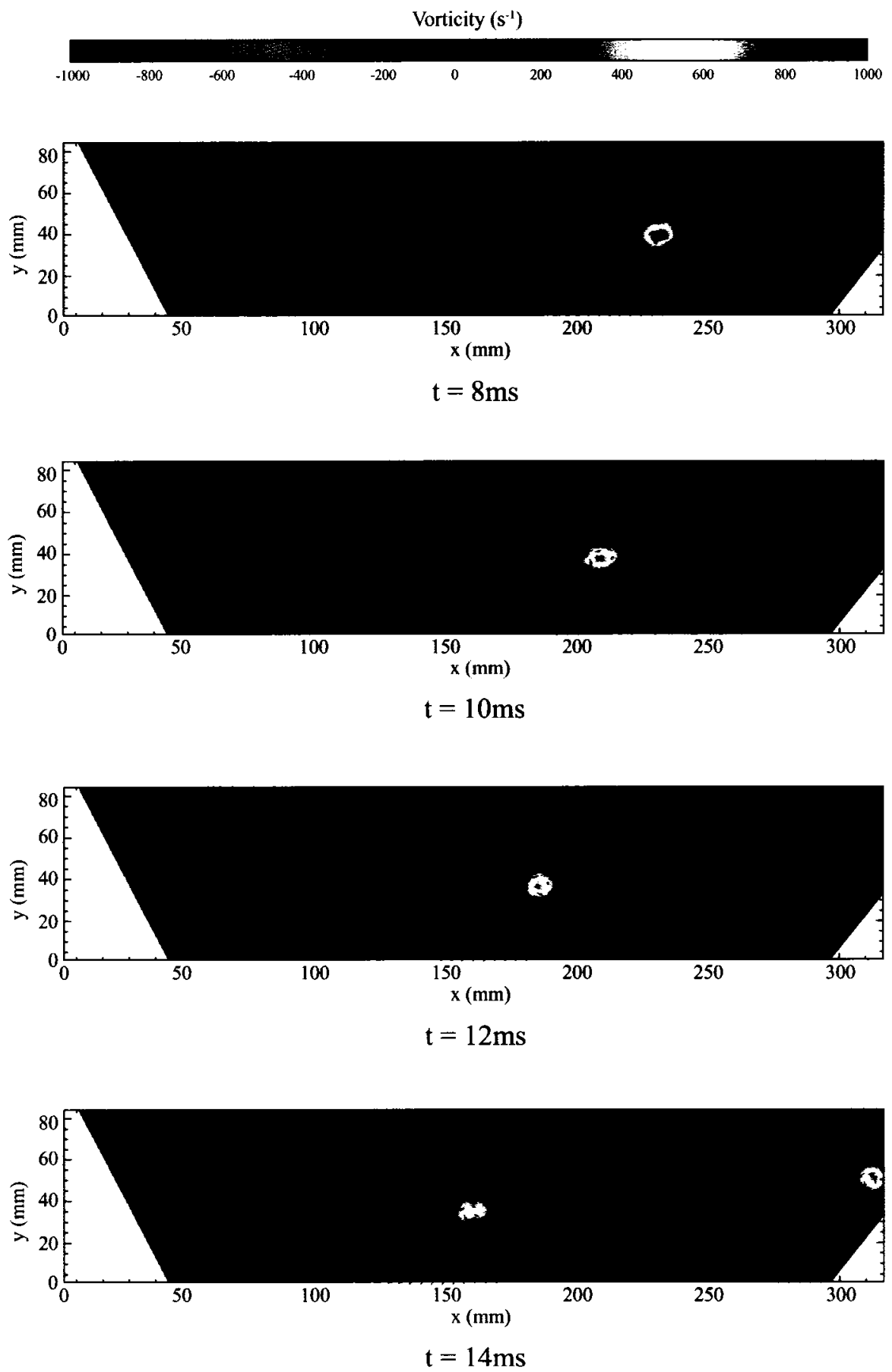

Figure 5.9: The vorticity in the flow for the free-vortex case, $8 \mathrm{~ms}$ through $14 \mathrm{~ms}$; equivalent to an approximate vortex miss-distance of $\infty$, with average-subtracted velocity vectors to demonstrate flow direction and relative magnitude. Measurement at a fan speed of $10 \mathrm{~Hz}$, which corresponds to a free-stream velocity of about $11 \mathrm{~m} / \mathrm{s}$. 

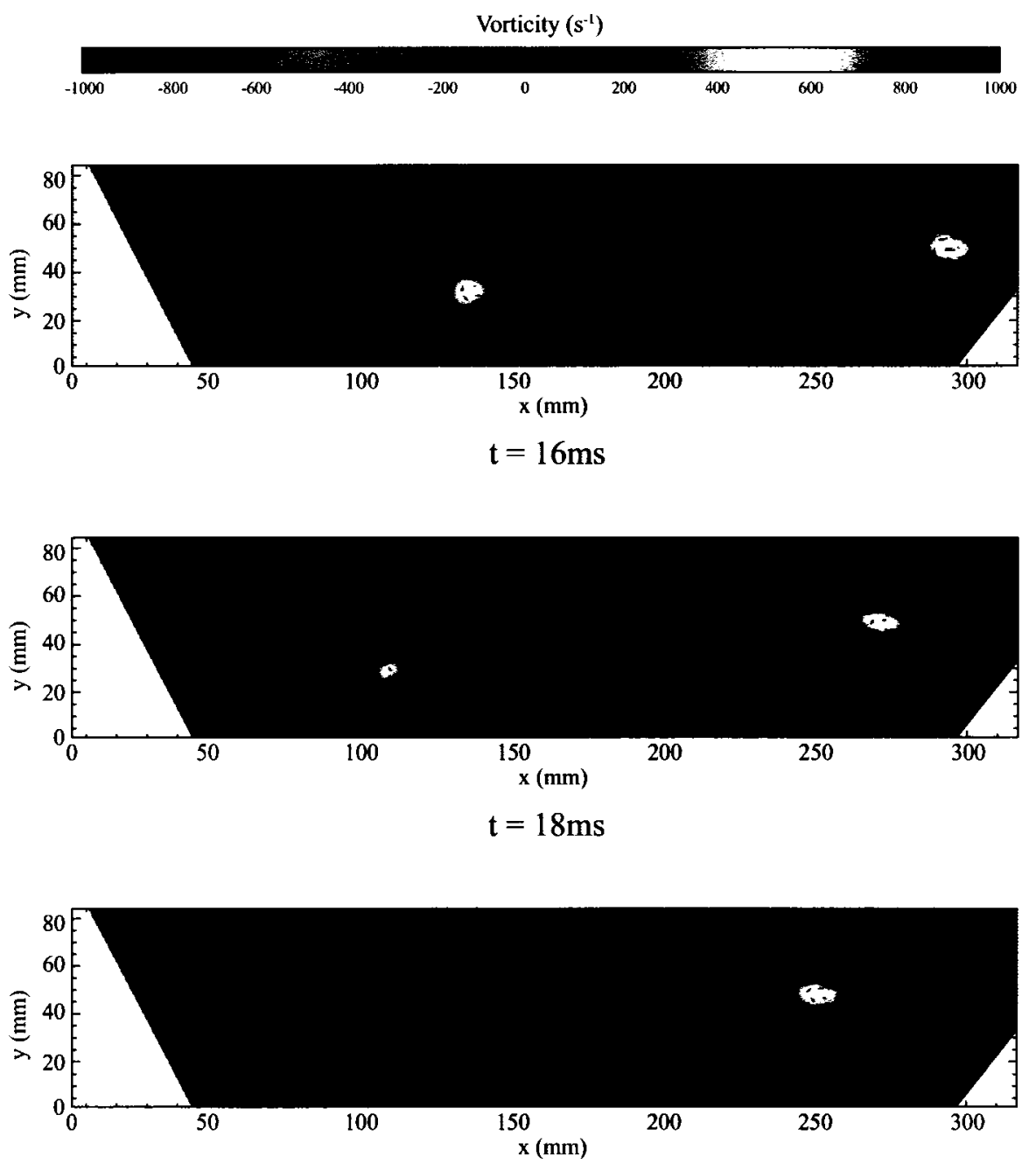

$\mathrm{t}=20 \mathrm{~ms}$

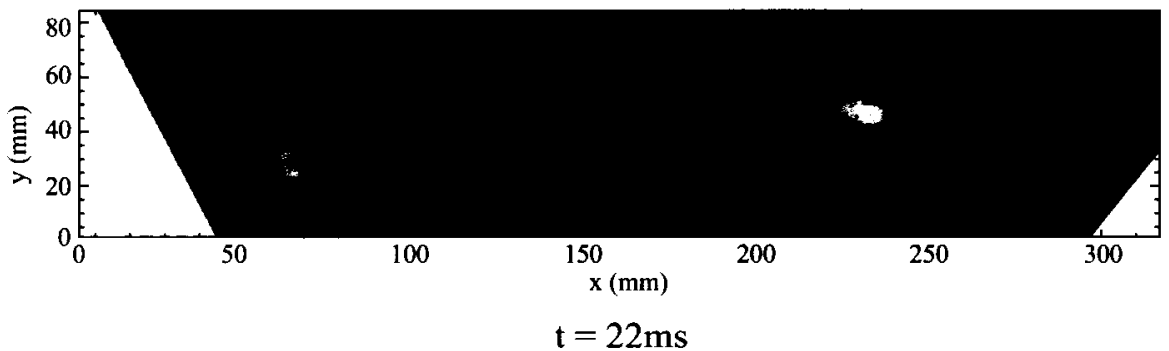

Figure 5.10: The vorticity in the flow for the free-vortex case, $16 \mathrm{~ms}$ through $22 \mathrm{~ms}$; equivalent to an approximate vortex miss-distance of $\infty$, with average-subtracted velocity vectors to demonstrate flow direction and relative magnitude. Measurement at a fan speed of $10 \mathrm{~Hz}$, which corresponds to a free-stream velocity of about $11 \mathrm{~m} / \mathrm{s}$. 
Velocity $(\mathrm{m} / \mathrm{s})$
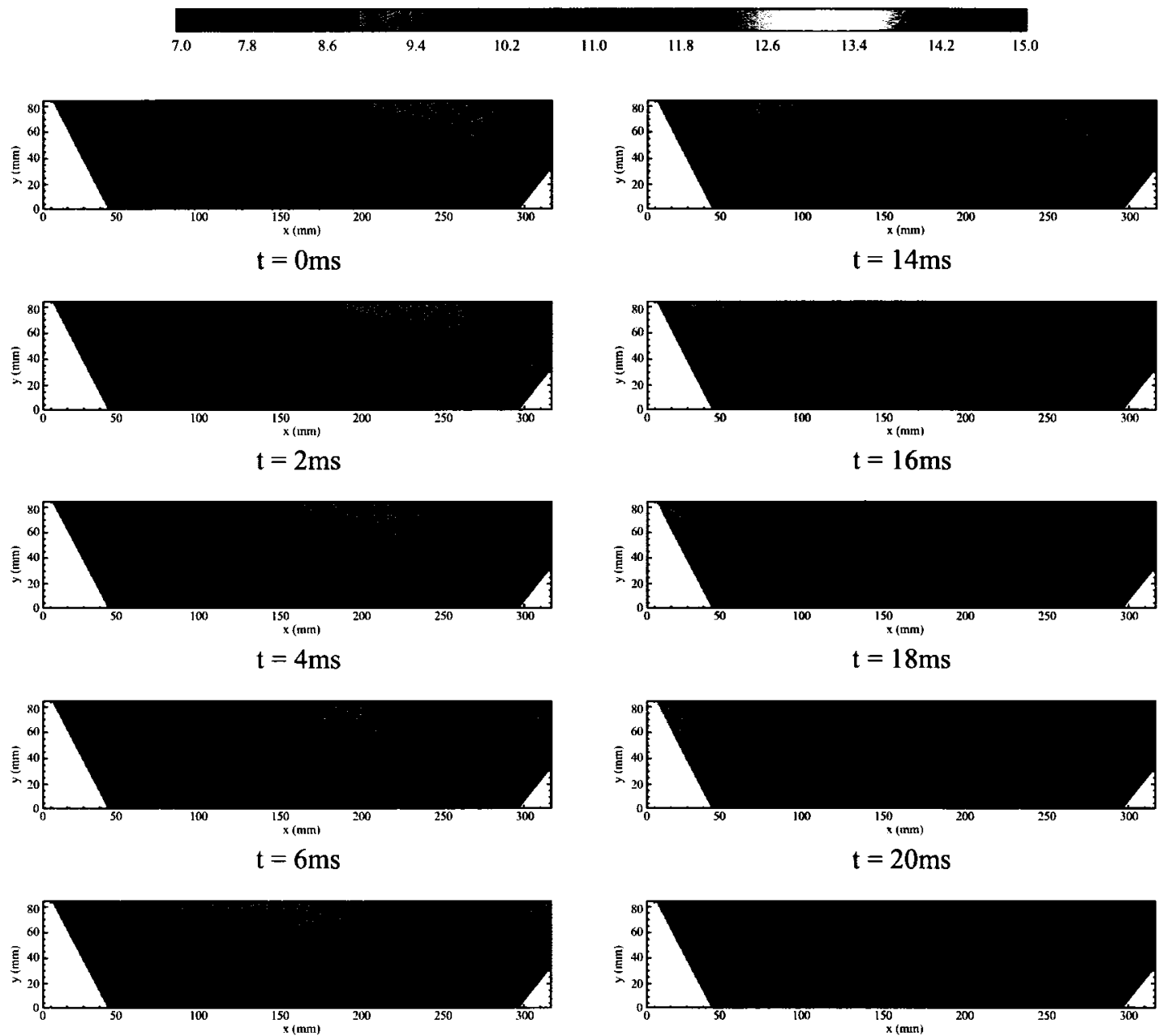

$\mathrm{t}=8 \mathrm{~ms}$

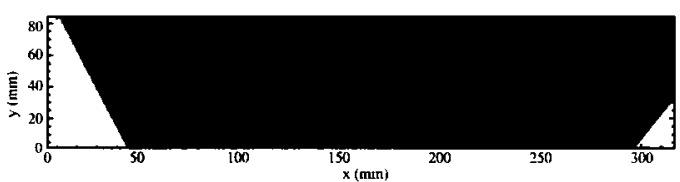

$\mathrm{t}=22 \mathrm{~ms}$

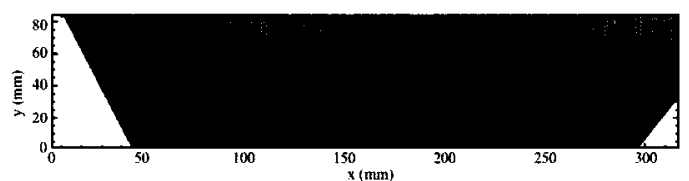

$\mathrm{t}=10 \mathrm{~ms}$

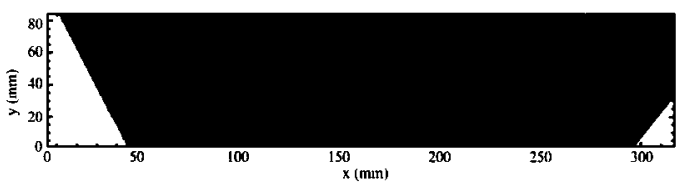

$\mathrm{t}=24 \mathrm{~ms}$

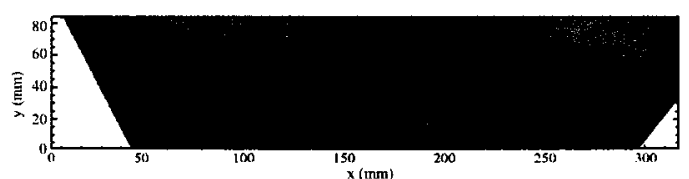

$\mathrm{t}=12 \mathrm{~ms}$

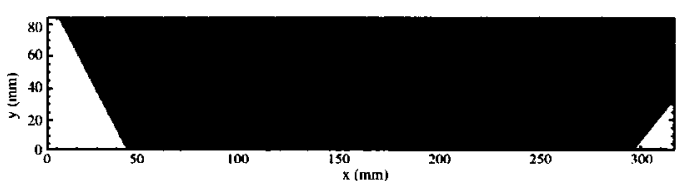

$\mathrm{t}=26 \mathrm{~ms}$

Figure 5.11: The velocity field in the flow for the free-vortex case; equivalent to an approximate vortex miss-distance of $\infty$. Measurement at a fan speed of $10 \mathrm{~Hz}$, which corresponds to a free-stream velocity of about $11 \mathrm{~m} / \mathrm{s}$. 

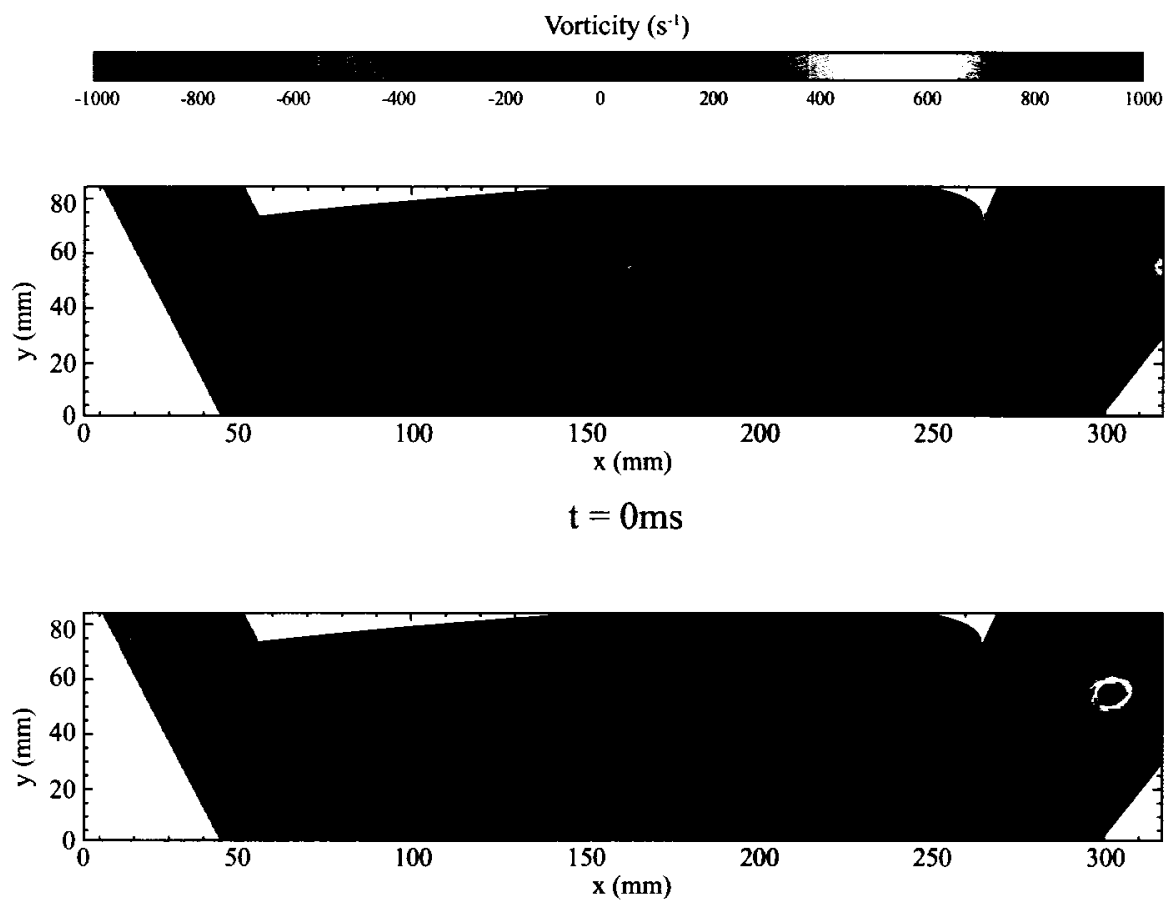

$\mathrm{t}=2 \mathrm{~ms}$

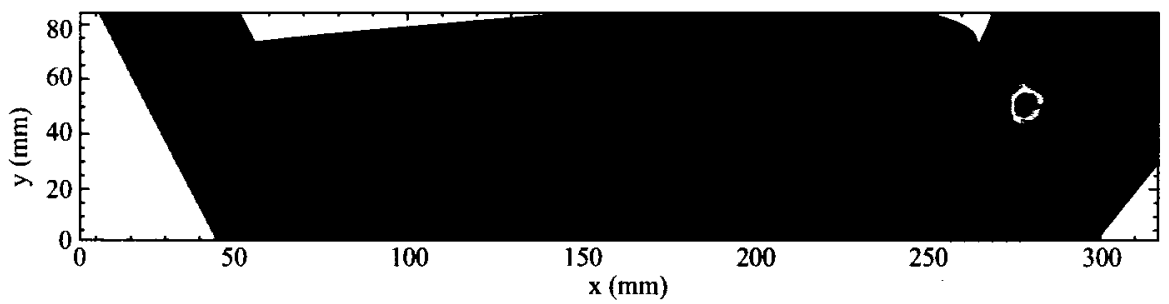

$\mathrm{t}=4 \mathrm{~ms}$

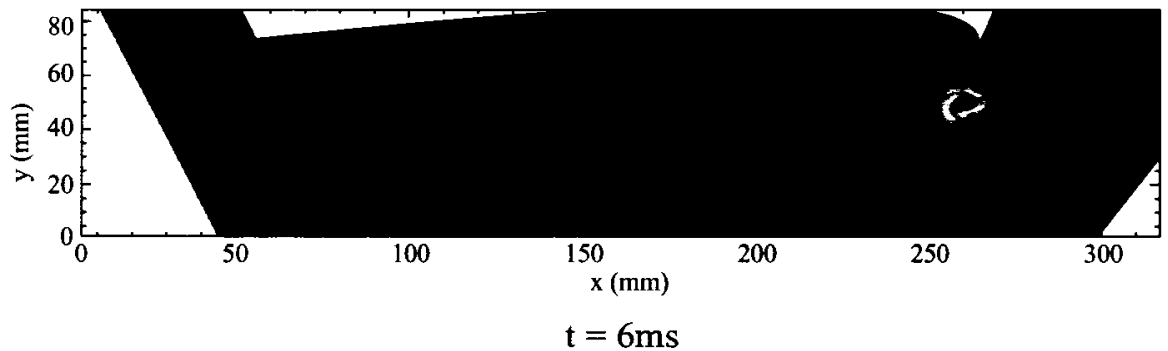

Figure 5.12: The vorticity in the flow for the vortex impaction on the NACA 0012 airfoil at zero angle of attack and a vortex miss-distance of $0.1 \bar{c}$, with average-subtracted velocity vectors to demonstrate flow direction and relative magnitude. Measurement at a fan speed of $10 \mathrm{~Hz}$, which corresponds to a free-stream velocity of about $11 \mathrm{~m} / \mathrm{s}$. 

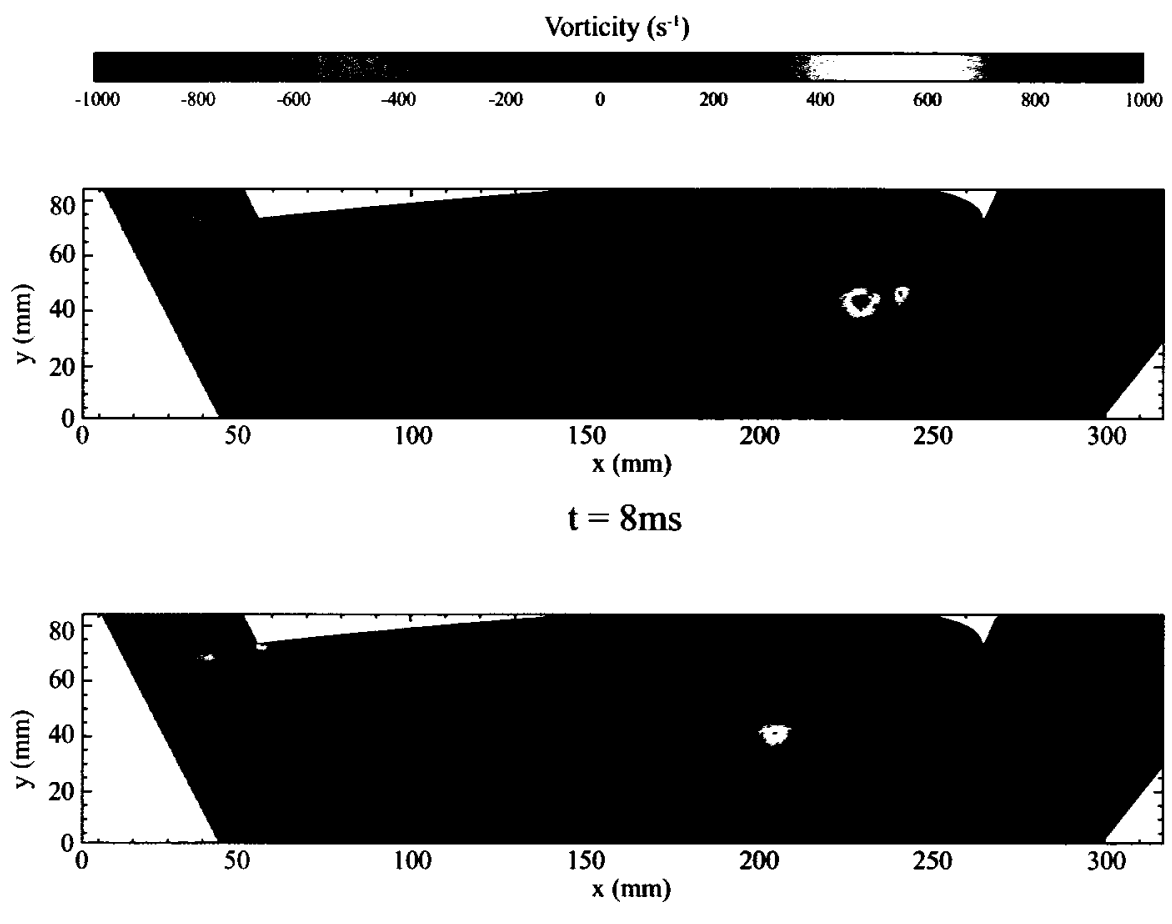

$\mathrm{t}=10 \mathrm{~ms}$

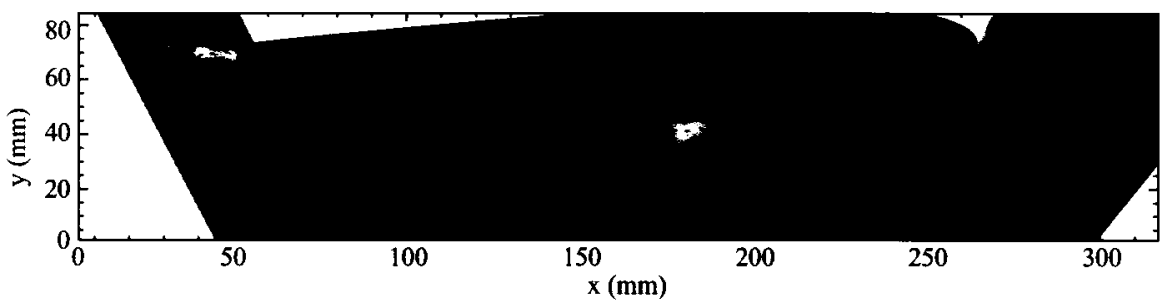

$t=12 \mathrm{~ms}$

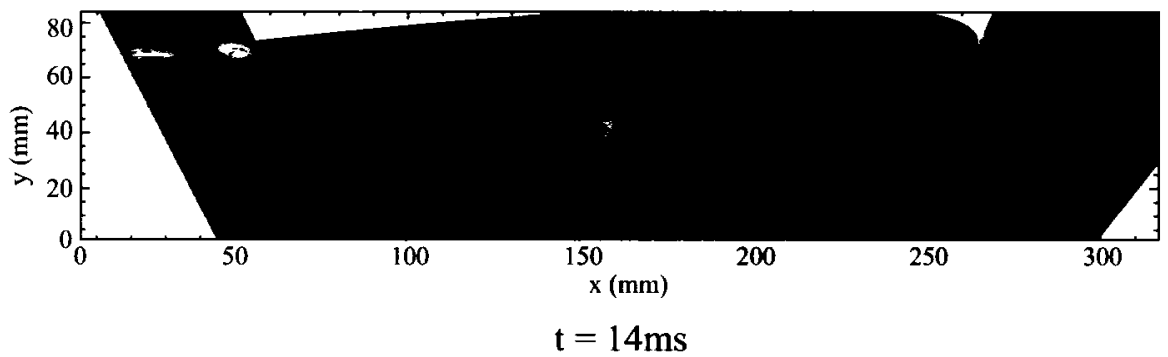

Figure 5.13: The vorticity in the flow, 0ms through $6 \mathrm{~ms}$, for the vortex impaction on the NACA 0012 airfoil at zero angle of attack and a vortex miss-distance of $0.1 \bar{c}$, with average-subtracted velocity vectors to demonstrate flow direction and relative magnitude. Measurement at a fan speed of $10 \mathrm{~Hz}$, which corresponds to a free-stream velocity of about $11 \mathrm{~m} / \mathrm{s}$. 

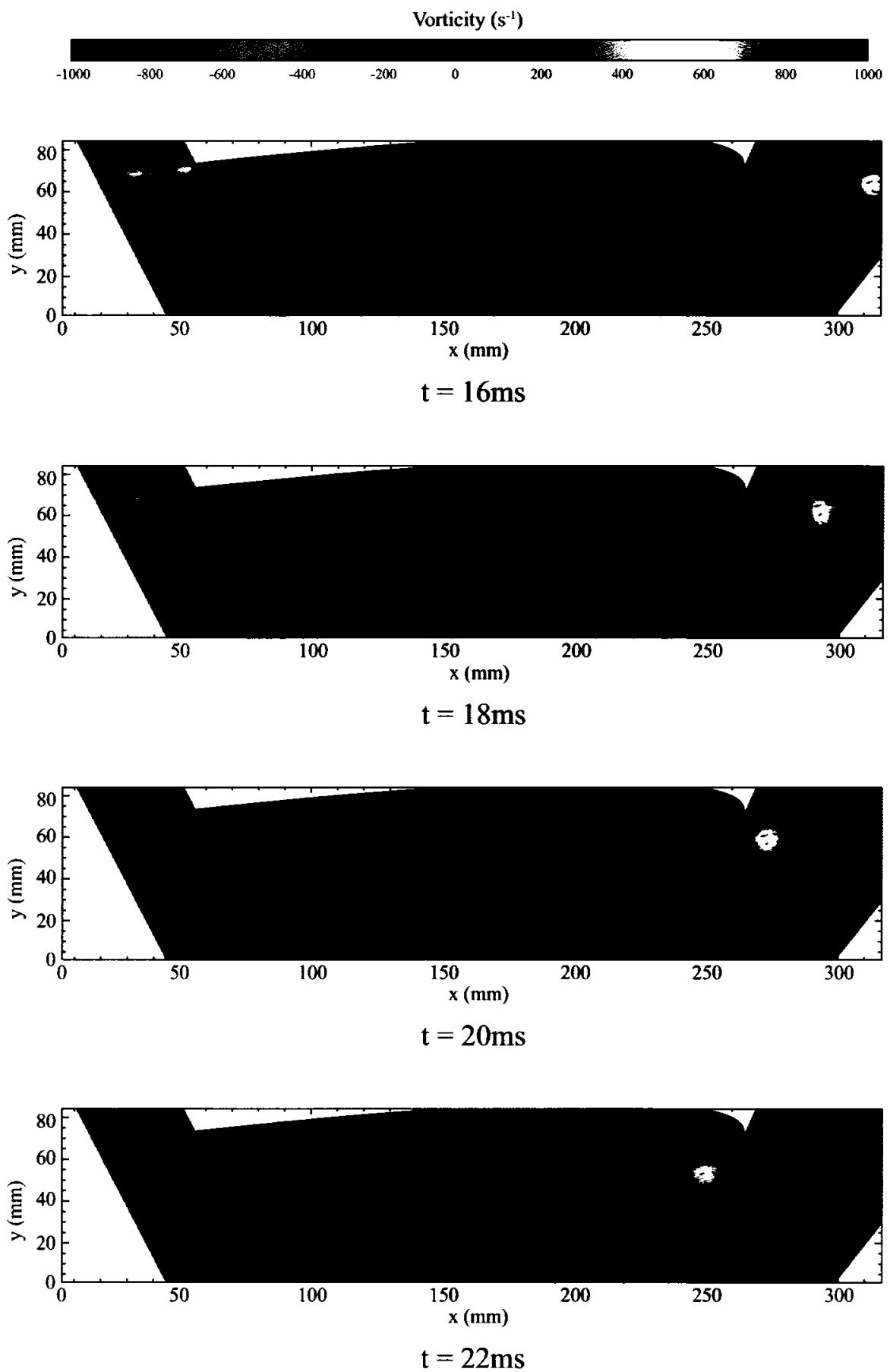

Figure 5.14: The vorticity in the flow, $8 \mathrm{~ms}$ through $14 \mathrm{~ms}$, for the vortex impaction on the NACA 0012 airfoil at zero angle of attack and a vortex miss-distance of $0.1 \bar{c}$, with average-subtracted velocity vectors to demonstrate flow direction and relative magnitude. Measurement at a fan speed of $10 \mathrm{~Hz}$, which corresponds to a free-stream velocity of about $11 \mathrm{~m} / \mathrm{s}$. 

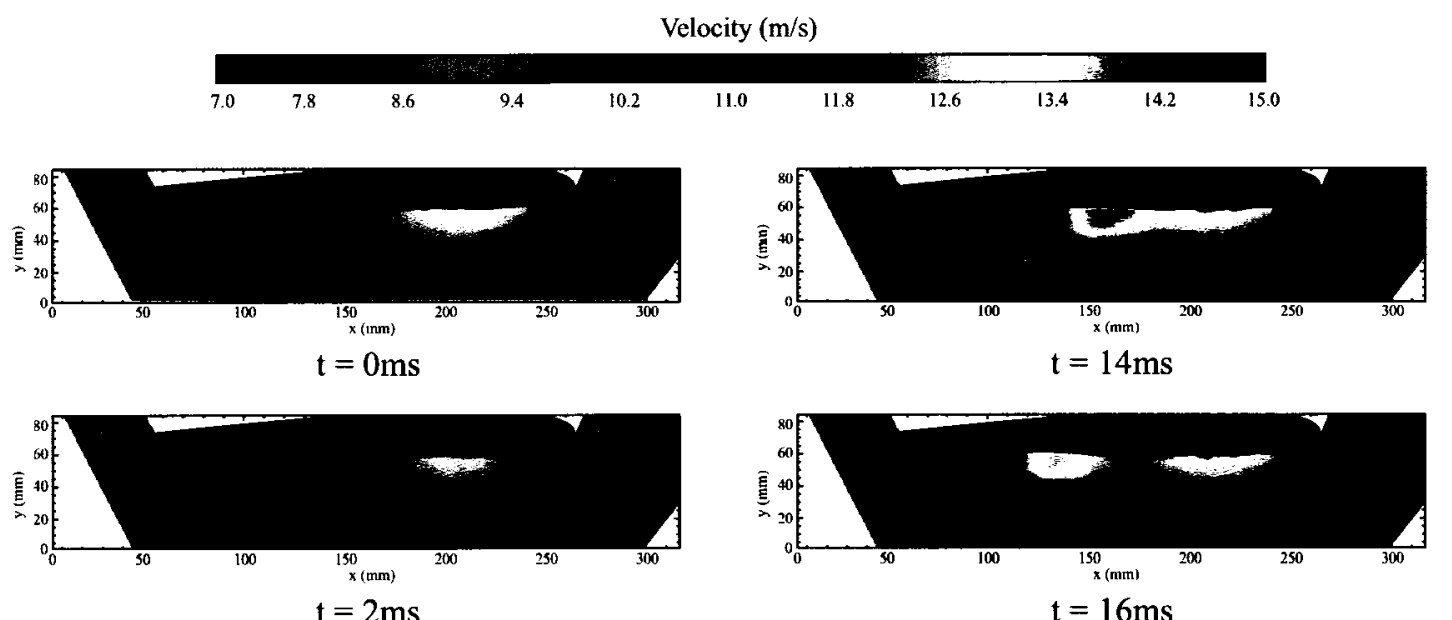

$\mathrm{t}=2 \mathrm{~ms}$
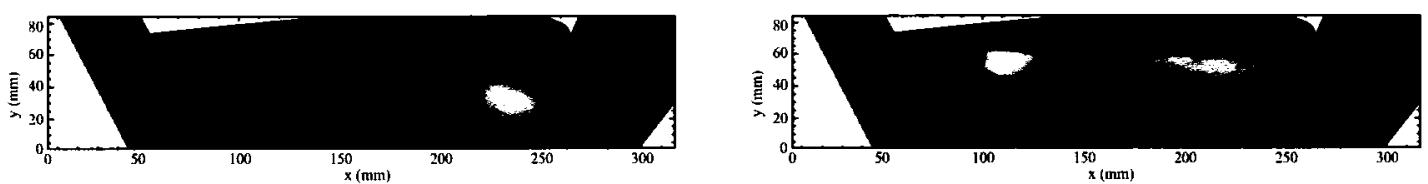

$\mathrm{t}=4 \mathrm{~ms}$
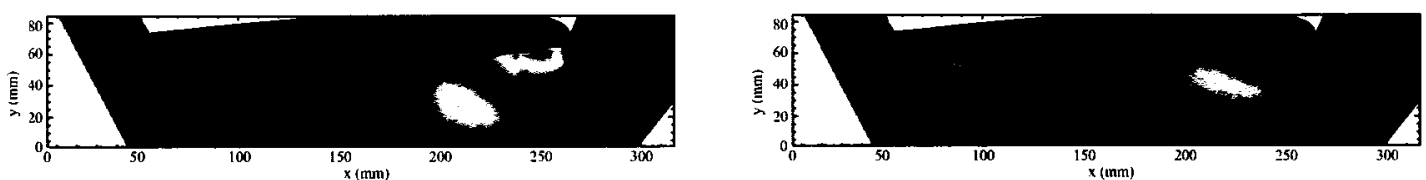

$\mathrm{t}=6 \mathrm{~ms}$

$\mathrm{t}=20 \mathrm{~ms}$
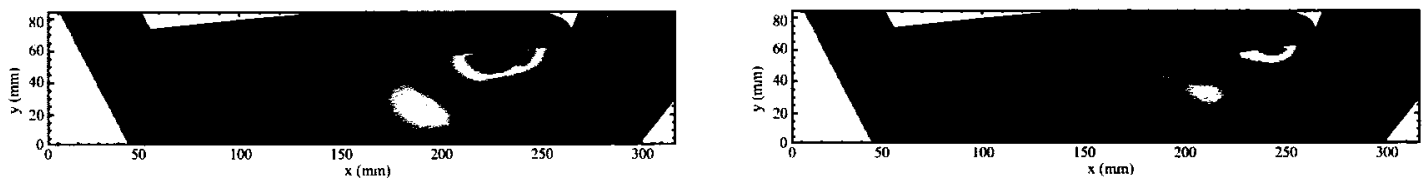

$\mathrm{t}=8 \mathrm{~ms}$

$\mathrm{t}=22 \mathrm{~ms}$
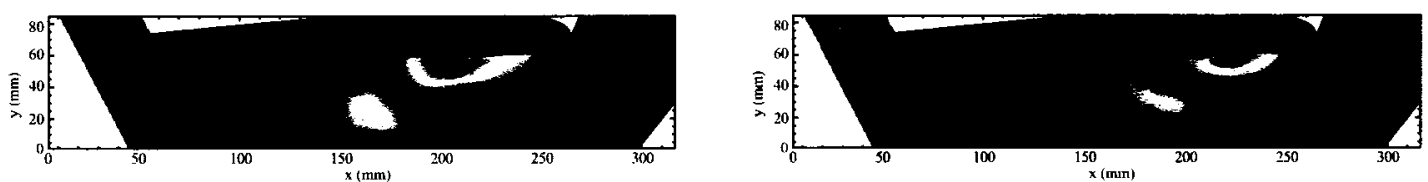

$\mathrm{t}=10 \mathrm{~ms}$

$\mathrm{t}=24 \mathrm{~ms}$

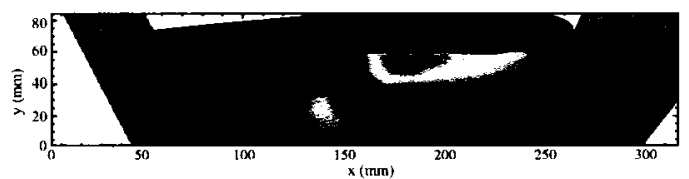

$\mathrm{t}=12 \mathrm{~ms}$

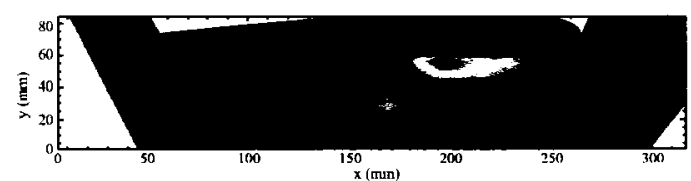

$\mathrm{t}=26 \mathrm{~ms}$

Figure 5.15: The velocity field in the flow, $16 \mathrm{~ms}$ through $22 \mathrm{~ms}$, for the vortex impaction on the NACA 0012 airfoil at zero angle of attack and vortex miss-distance of $0.1 \bar{c}$. Measurement at a fan speed of $10 \mathrm{~Hz}$, which corresponds to a free-stream velocity of about $11 \mathrm{~m} / \mathrm{s}$. 


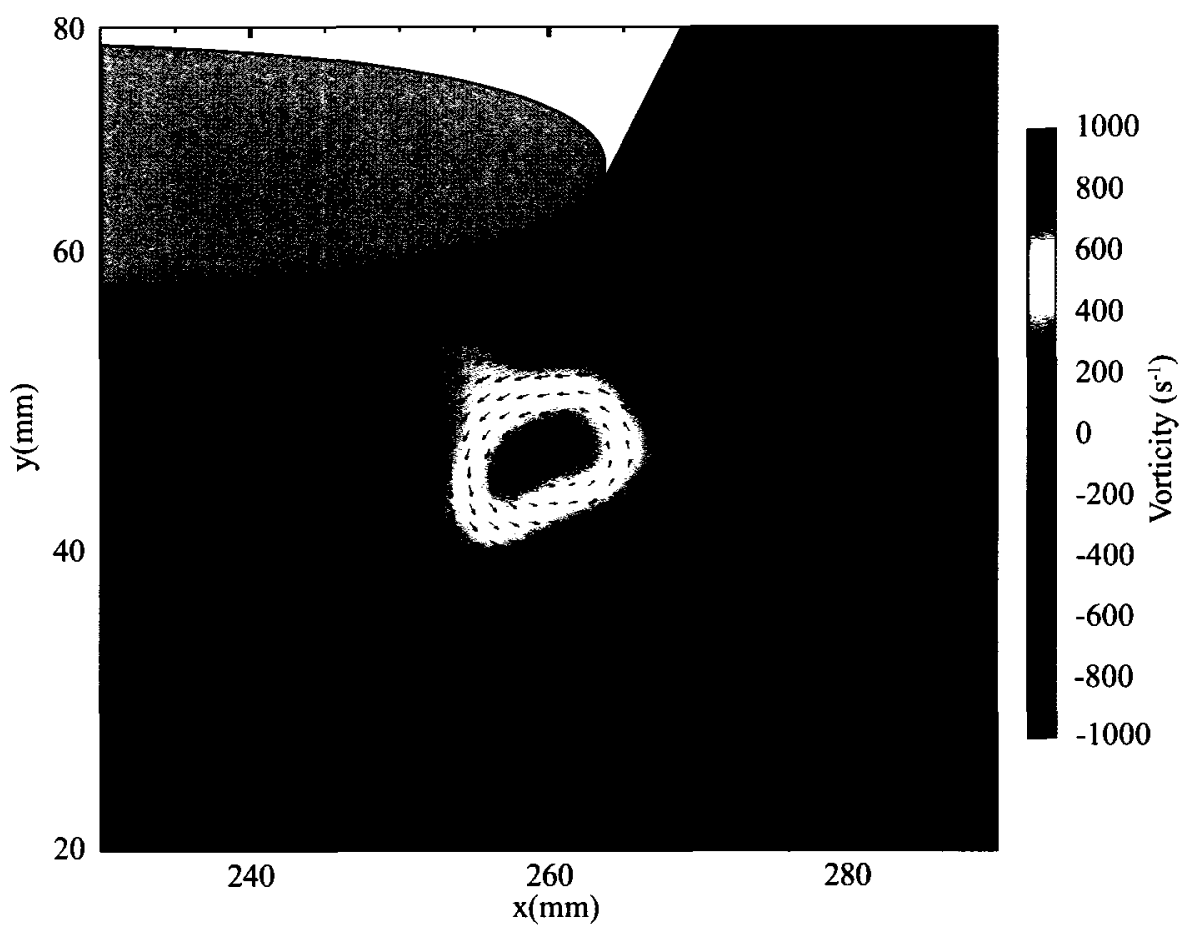

Figure 5.16: The airfoil-vortex interaction in high vector resolution. 


\section{Chapter 6}

\section{Conclusions}

A state-of-the-art particle image velocimetry system was installed and brought to functionality within a two-dimensional wind tunnel designed specifically for PIV measurements of airfoil-vortex interactions. The installation of the PIV system included the mounting of the laser and camera at the test section of the wind tunnel to allow for the measurements to be made. Also, a tracer particle generation unit was purchased and installed in the wind tunnel, with the appropriate distribution mechanism. Modifications to the aerosol tracer particle generator were made in order to reduce the particle size to an acceptable diameter. System optimization and sensitivity analyses were conducted to yield appropriate testing and analysis parameters for different flow situations.

In addition to this, two separate vortex generating units were designed, constructed and implemented in the wind tunnel. Through testing, their respective vortex creation capabilities were investigated. A test rotor, the blade which was to interact with an incoming vortex in the blade-vortex interaction, was designed and fabricated using raw materials and a computer numerically controlled mill. The test rotor was also integrated into the wind tunnel at the appropriate locations for the BVI measurements.

Measurements were conducted to showcase the PIV system's capabilities in the modified wind tunnel to successfully make BVI measurements under a variety of user controlled conditions. Since the wind tunnel will likely undergo turbulence testing with a hot wire anemometry (HWA) system in the near future, turbulence estimations were made in the wake of the vortex generating airfoil such that the turbulence measuring capabilities of the 
PIV system can be quantified when these highly accurate HWA results are available. The simple case of the free flow around a NACA 0012 airfoil were also completed, given the vast amount of data, experimental and numerical, which this can be compared to.

Finally, measurements of a free vortex in the flow were made, in order to characterize the vortex which was shed from the vortex generating unit and used in the airfoil-vortex measurements. This lead to the measurements of an airfoil-vortex interaction at one miss distance, a variable which can be altered to the experimenter's desire. 


\section{List of References}

[1] I. Grant, "Particle image velocimetry: A review," Proceedings of the Institution of Mechanical Engine, vol. 211, pp. 55-76, 1997.

[2] M. Raffel, C. Willert, and J. Kompenhans, Particle Image Velocimetry: A Practical Guide. Berlin: Springer, 1998.

[3] R. B. Green, C. J. Doolan, and R. M. Cannon, "Measurments of the orthogonal bladevortex interaction using a particle image velocimetry technique," Experiments in Fluids, vol. 29, pp. 369-379, 2000.

[4] M. Raffel, H. Richard, K. Ehrenfried, B. V. der Wall, C. Burley, P. Beaumier, K. McAlister, and K. Pengel, "Recording and evaluation methods of PIV investigations on a helicopter rotor model," Experiments in Fluids, vol. 36, pp. 146-156, 2004.

[5] T. Berenger, D. Favier, C. Maresca, and E. Berton, "Experimental and numerical investigation of rotor aerodynamics in forward flight," Journal of Aircraft, vol. 34, no. 3 , pp. 394-399, 1997.

[6] G. R. Srinivasan, "Aerodynamics of two-dimensional blade-vortex interaction," AIAA Journal, vol. 24, no. 10, pp. 1569-1576, 1986.

[7] B. W. Sim and F. H. Schmitz, "Blade vortex interaction (BVI) noise: Retreating side characteristics, sensitivity to chordwise loading and unsteady aerodynamics," in American Helicopter Society (AHS) Aerome-chanics Specialists Meeting, (Atlanta, Georgia), November 2000.

[8] J. Westerweel, "Fundamentals of digital particle image velocimetry," Measurement Science and Technology, vol. 8, pp. 1379-1392, 1997.

[9] C. E. Willert and M. Gharib, "Digital particle image velocimetry," Experiments in Fluids, vol. 10, pp. 181-193, 1991.

[10] R. D. Keane and R. J. Adrian, "Theory of cross-correlation analysis of PIV images," Applied Scientific Research, vol. 49, pp. 191-215, 1992. 
[11] O. Pust, "PIV: Direct cross-correlation compared with FFT-based cross-correlation," in $10^{\text {th }}$ International Symposium on Applications of Laser Techniques to Fluid Mechanics, (Lisbon, Portugal), July 2000.

[12] A. Melling, "Tracer particles and seeding for particle image velocimetry," Measurement Science and Technology, vol. 8, pp. 1406-1416, 1997.

[13] G. H. Smith, I. Grant, A. Liu, D. Infield, and T. Eich, "A wind tunnel examination of vortices shed from a wind generator using particle image velocimetry," in $6^{\text {th }}$ International Symposium on Applications of Laser Techniques to Fluid Mechanics, (Lisbon, Portugal), July 1992.

[14] Dantec Measurement Technology A/S, P.O. Box 121, Tonsbakken 18, DK-2740 Skovlunde, Denmark, FlowMap Particle Image Velocimetry Instrumation: Installation E User's Guide, fifth ed., 2000.

[15] J. Westerweel, D. Dabiri, and M. Gharib, "The effect of a discrete window offset on the accuracy of cross-correlation analysis of digital PIV recordings," Experiments in Fluids, vol. 23, pp. 20-28, 1997.

[16] D. P. Hart, "PIV error correction," Experiments in Fluids, vol. 29, pp. 13-22, 2000.

[17] K. Anandarajah, G. K. Hargrave, and N. A. Halliwell, "Digitl particle image velocimetry: Partial image error," Journal of Physics: Conference Series, vol. 45, pp. 174-185, 2006.

[18] J. Westerweel, "Efficient detection of spurious vectors in particle image velocimetry data," Experiments in Fluids, vol. 16, pp. 236-247, 1994.

[19] J. Westerweel and F. Scarano, "Universal outlier detection for PIV data," Experiments in Fluids, vol. 39, p. 10961100, 2005.

[20] H. T. Huang, H. E. Fiedler, and J. J. Wang, "Limitation and improvement of PIV Part I," Experiments in Fluids, vol. 15, pp. 168-174, 1993a.

[21] H. T. Huang, H. E. Fiedler, and J. J. Wang, "Limitation and improvement of PIV Part II," Experiments in Fluids, vol. 15, pp. 263-273, 1993b.

[22] J. Nogueira, A. Lecuona, and P. A. Rodríguez, "Data validation, false vectors correction and derived magnitudes calculation on PIV data," Measurement Science and Technology, vol. 8, p. 14931501, 1997.

[23] M. L. R. F. Scarano, "Iterative multigrid approach in PIV image processing with discrete window offset," Experiments in Fluids, vol. 26, pp. 513-523, 1999.

[24] D. P. Hart, "Super-resolution PIV by recursive local-correlation," Journal of Visualization, vol. 10, pp. 1-10, 1999. 
[25] R. D. Keane and R. J. Adrian, "Prospects for super-resolution with particle image velocimetry," Optical Diagnostics in Fluid and Thermal Flow, vol. 2005, p. 283295, 1993.

[26] R. D. Keane, R. J. Adrian, and J. Zhang, "Super-resolution particle-imaging velocimetry," Measurement Science and Technology, vol. 6, p. 754768, 1995.

[27] R. J. Perkins and J. C. R. Hunt, "Particle tracking in turbulent flows," Advances in Turbulence, vol. 2, p. 286291, 1989.

[28] Y. G. Guezennec and N. Kiritsis, "Statistical investigation of errors in particle image velocimetry," Experiments in Fluids, vol. 10, p. 138146, 1990.

[29] W. Wenguo, F. Weicheng, L. Guangxuan, and Q. Jun, "An improved cross-corelation method for paticle image velocimetry," Acta Mechanica Sinica, vol. 17, no. 4, pp. 332$339,2001$.

[30] R. J. Adrian, "Dynamic ranges of velocity and spatial resolution of particle image velocimetry," Measurement Science and Technology, vol. 8, pp. 1393-1398, 1997.

[31] J. Bolinder, "On the accuracy of a digital particle image velocimetry system," tech. rep., Institutionen för Värme- och Kraftteknik, 1999.

[32] J. Westerweel, "Theoretical analysis of the measurments precision in particle imave velocimetry," Experiments in Fluids (Suppl.), pp. S3-S12, 2000.

[33] D. D. Seath, J.-M. Kim, and D. R. Wilson, "Investigation of the parallel blade-vortex interaction at low speed," Journal of Aircraft, vol. 26, no. 4, pp. 328-333, 1989.

[34] H. Gläßel, V. Klöppel, and S. Rudolph, "Neural control of helicopter blade-vortex interaction noise," in SPIE $8^{\text {th }}$ International Symposium on Smart Structures and $M a-$ terials, (Newport Beach, CA), March 2001.

[35] J. L. Tangler, "Schlieren and noise studies of rotors in forward flight," in $33^{\text {rd }}$ American Helicopter Society forum, (Washington, DC), 1977.

[36] S. Lee and D. Bershader, "Head-on parallel blade-vortex interaction," AIAA Journal, vol. 32, no. 1, pp. 16-22, 1994.

[37] Y. H. Yu, "Rotor blade-vortex interaction noise," Progress in Aerospace Sciences, vol. 36, pp. 97-115, 2000.

[38] A. R. George and Sim, "Tilt-rotor blade-vortex interaction noise," tech. rep., NASA Langley Research Center, 1996.

[39] F. H. Schmitz and B. W. Sim, "Radiation and directionality characteristics of advancing Side blade-vortex interaction (BVI) noise," in $6^{\text {th }} I A A / C E A S$ Aeroacoustics Conference, (Lahaina, Hawaii), June 2000. 
[40] F. M. White, Fluid Mechanics. McGraw-Hill, $4^{\text {th }}$ ed., 1999.

[41] G. Rahier, Modeling of airfoil-vortex interaction and application to a helicopter; contribution to blade-vortex interaction noise prediction. $\mathrm{PhD}$ thesis, Office National d'Etudes et de Recherches Aerospatiales, 1997.

[42] C. Tung, Y. H. Yu, and S. L. Low, "Aerodynamic aspects of blade-vortex interaction (BVI)," in $27^{\text {th }}$ Fluid Dynamics Conference, (New Orleans, LA), June 1996.

[43] S.-H. Wong, M. Papadakis, L. S. Nizampatham, and K. A. Hoffmann, "Computational investigation of blade vortex interaction noise," in $38^{\text {th }}$ Aerospace Sciences Meeting and Exhibit, (Reno, NV), January 2000.

[44] W. S. Oh, J. S. Kim, and O. J. Kwon, "Numerical simulation of two-dimensional bladevortex interactions using unstructured adaptive meshes," AIAA Journal, vol. 40, no. 3 , pp. $474-480,2002$.

[45] M. Mamou, M. Khalid, and H. Xu, "Unsteady flows past two airfoils in tandem and airfoil-vortex interaction," Canadian Aeronautics and Space Journal, vol. 47, no. 4, pp. 357-366, 2001.

[46] S. Y. Lin and Y. F. Chen, "Numerical study of head-on blade-vortex interaction noise," in AIAA, Aerospace Sciences Meeting $\mathcal{G}$ Exhibit, 35th, (Reno, NV), January 1997.

[47] S. Lee, "Reduction of blade-vortex interaction noise through porous leading edge," AIAA Journal, vol. 32, no. 3, pp. 480-488, 1994.

[48] Y. H. Yu, "Miss distance for rotor blade-vortex interaction noise reduction," in $2^{\text {nd }}$ Aeroacoustics Conference, (State College, PA), May 1996.

[49] J. Straus, P. Renzoni, and R. E. Mayle, "Airfoil pressure measurements during a blade vortex interaction and a comparison with theory," AIAA Journal, vol. 28, no. 2, pp. 222-228, 1990.

[50] I. M. Kalkhoran and D. R. Wilson, "Experimental investigation of the parallel vortexairfoil interaction at transonic speeds," AIAA Journal, vol. 30, no. 8, pp. 2087-2092, 1992.

[51] E. R. Booth and J. C. Yu, "Experimental observations of two-dimensional blade-vortex interaction," AIAA Journal, vol. 28, no. 8, pp. 1353-1359, 1990.

[52] E. R. Booth and J. C. Yu, "Two-dimensional blade-vortex flow visualization investigation," AIAA Journal, vol. 24, no. 9, pp. 1468-1473, 1986.

[53] F. X. Caradonna and R. C. Strawn, "An experimental and computational study of rotor-vortex interactions," Vertica, vol. 12, no. 4, pp. 315-327, 1988. 
[54] C. J. Doolan, F. N. Coton, and R. A. M. Galbraith, "Measurement of three-dimensional vortices using, a hot wire anemometer," in $30^{\text {th }}$ AIAA Fluid Dynamics Conference, (Norfolk, VA), June 1999.

[55] E. Malakiel, V. Levinski, and J. Cohen, "The evolution of a localized vortex disturbance in external shear flows. part 2. comparison with experiments in rotating shear flows," Journal of Fluid Mechanics, vol. 397, pp. 351-380, 1999.

[56] M. B. Horner, R. A. M. Galbraith, F. N. Coton, J. N. Stewart, and I. Grant, "Examination of vortex deformation during blade-vortex interaction," AIAA Journal, vol. 34, no. 6, pp. 1188-1194, 1996.

[57] I. Grant, X. Pan, X. Wang, and N. Stewart, "Correction for viewing angle applied to PIV data obtained in aerodynamic blade vortex interaction studies," Experiments in Fluids, vol. 18, pp. 95-99, 1994.

[58] M. Green, K. Parker, and J. Soria, "2D DPIV of a pitching aerofoil," in $4^{\text {th }}$ Australian Conference on Laser Diagnostics in Fluid Mechanics and Combustion, pp. 53-56, 2005.

[59] I. Grant and P. Parkin, "A DPIV study of the trailing vortex elements from the blades," Experiments in Fluids, vol. 28, pp. 368-376, 2000.

[60] W. J. Devenport, M. C. Rife, S. I. Liapis, and G. J. Follin, "The structure and development of a wing-tip vortex," Journal of Fluid Mechanics, vol. 312, pp. 67-106, 1996.

[61] D. Brassard, "A 2D transverse vortex wind tunnel for PIV investigation of airfoil vortex interaction," Master's thesis, Carleton University, Department of Mechanical and Aerospace Engineering, Ottawa, Ontario, Canada, 2006. 


\section{Appendix A}

\section{MatLab Post Processing Tools Code}

In addition to the available post processing tools packaged with Dantec's FlowMap software, tools for handling raw PIV data were programmed using MatLab. These tools allowed for simple importing and plotting (various formats) of the raw PIV vector velocity data which was exported from the PIV measurement software along with averaging and spurious vector identification. Also, since the software does not allow for the creation of compilations of numerous measurement areas into one large measurement (such as combining many close measurements of the airfoils surface into one large measurement), the tools were generated with MatLab with creating such figures from numerous raw data files in mind. The MatLab code snippets are included below: 


\section{Loading Raw Data Files}

function vect_map $=$ read_dat (file_name) $\% \% \% \% \% \% \% \% \% \% \% \% \% \% \% \% \% \% \% \% \% \% \% \% \% \% \% \% \% \% \% \% \% \% \% \% \% \% \% \% \% \% \% \% \% \% \% \% \% \% \% \% \% \% \% \% \% \% \% \% \% \% \% \% \% \% \%$

$\%$ Wes Burwash

$\% \quad$ Carleton University BVI PIV

$\%$ Rotorcraft Group

October 5, 2006

PART OF: PIV Post Processing Tools

Function Description:

This funtion takes in a text-base PIV data file with one header row and organizes the flow field information into its respective $X, Y, U$ and $V$ components. The $\mathrm{X}, \mathrm{Y}, \mathrm{U}$ and $\mathrm{V}$ vectors are then transformed into matricies for ease of use later. The input is the file name for the data file holding the PIV data and the output is the $X, Y, U$ and $V$ matricies which describe the flow field.

vect_map = read_dat (file_name)

Input:

$\%$ file_name : The full filename of the data file you with to have Output : Matlab read in.

$\% \quad$ vect_map : A $4 \times 2 \times 2$ matrix. Essentially 4 matricies of the $\%$ location in $x$ and $y$, and the velocities in $u$ and $v$ $\% \quad$ Import the text fil

$[x, y, u, v]=$ textread (file_name, $\%$ f $\%$ f $\%$ f $\%$ ', 'headerlines',1)

$\% \quad$ Assemble the $X, Y$ grid after determining the size of the flow field

$\%$ region.

tot_rows $=$ length $(x)$;

$\mathrm{a}=0$;

$\mathrm{b}=1 ;$

$i=0$;

while $(a<b)$

$i=i+1$;

$a=x(i)$;

end

$b=x(i+1)$

clear a;

clear b;

rows $=i$

clear i;

cols = tot_rows / rows;

$\mathrm{X}=\mathrm{x}(1$ :rows $)$;

$\mathrm{Y}=\mathrm{y}(1$ :rows:tot_rows $)$;

$[X, Y]=$ meshgrid $(X, Y)$;

$\% \quad$ Assemble the U, $V$ flow field grid to lay upon the $X, Y$ grid for

$\% \quad$ plotting and furter calculations

for ( $i=1$ :rows)

for $(j=1: \operatorname{cols})$

$U(i, j)=u((j-1) *$ rows $+i)$

end

$v(i, j)=v((j-1) *$ rows $+i)$

end

$\mathrm{U}=\operatorname{transpose}(\mathrm{U})$

$\mathrm{V}=\operatorname{transpose}(\mathrm{V})$

$\%$ Assemble into one variable

vect_map $(1,:,:)=X(:,:)$;

vect_map $(2,:,:)=Y(:,:)$;

vect_map $(3,:,:)=U(:,:)$;

vect_map $(4,:,:)=\mathrm{V}(:,:)$;

$\%$ End of file 


\section{Averaging a Set of Data Files}

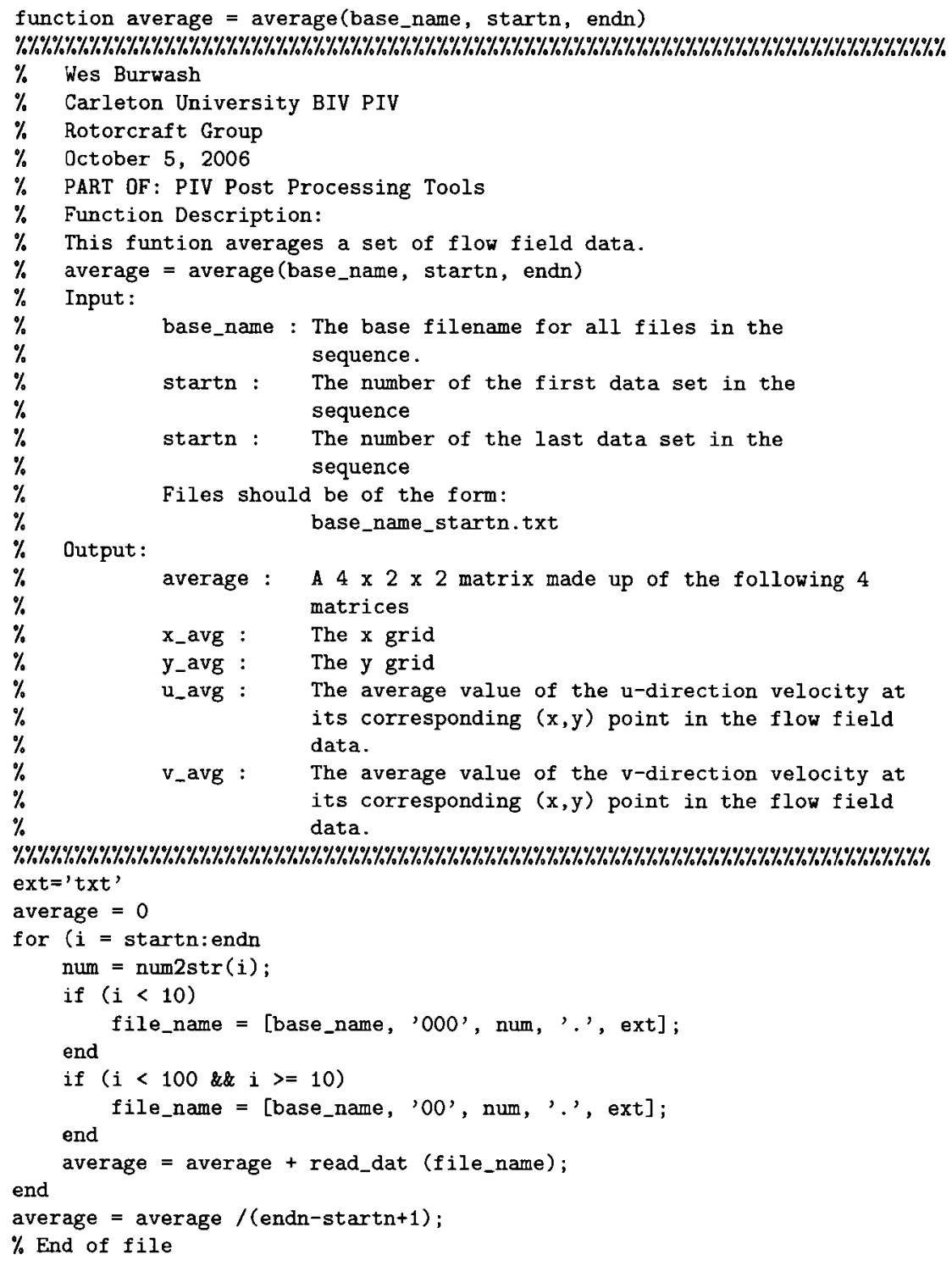




\section{Thin out Vector Field}

function [out_vect] $=$ thin (in_vect, factor) $\% \% \% \% \% \% \% \% \% \% \% \% \% \% \% \% \% \% \% \% \% \% \% \% \% \% \% \% \% \% \% \% \% \% \% \% \% \% \% \% \% \% \% \% \% \% \% \% \% \% \% \% \% \% \% \% \% \% \% \% \% \% \% \% \%$

$\%$ Wes Burwash

$\%$ Carleton University BVI PIV

$\%$ Rotorcraft Group

$\%$ October 5,2006

$\% \quad$ PART OF : PIV Post Processing Tools

$\%$ Function Description:

$\%$ This function takes in a two-dimensional matrix of flow field data and

$\%$ thins the number of elements by 'factor'. This makes for easier veiwing

$\%$ of the PIV data in vector plot form.

$\%$ [out_vect] $=$ thin2d (in_vect, factor)

$\% \quad$ Input:

$\% \quad$ in_vect : A $4 \times 2 \mathrm{D}$ matrix of values (usually coordinaltes or velocities)

$\%$ factor: The factor by which you wish to reduce the number $\% \quad$ of vectors in each direction. A factor of ' 2 ' will $\% \quad$ lead to 4 times less vectors $\left(2^{\sim} 2\right)$, while a factor of 3 will lead to nine times less $\left(3^{\wedge} 2\right)$.

$\%$ Output :

$\% \quad$ out_vect : The 'thinned' matrix with (factor)`2 less elements $\% \% \% \% \% \% \% \% \% \% \% \% \% \% \% \% \% \% \% \% \% \% \% \% \% \% \% \% \% \% \% \% \% \% \% \% \% \% \% \% \% \% \% \% \% \% \% \% \% \% \% \% \% \% \% \% \% \% \% \% \% \% \% \% \% \% \% \% \% \% \% \% \% \% \%$ $\%$ Determine size of input

[four, rows, cols] = size (in_vect);

$\%$ Thin out the elements

for $(k=1$ : four)

for ( $i=1$ :rows $/$ factor)

for $(j=1:$ cols/factor $)$ out_vect $(k, i, j)=$ in_vect $(k$, factor*i,factor $* j)$;

end

end

end

\section{Plot Velocity Vectors}

function vecplot(vector_map, factor, plot_title) \%\%\%\%\%\%\%\%\%\%\%\%\%\%\%\%\%\%\%\%\%\%\%\%\%\%\%\%\%\%\%\%\%\%\%\%\%\%\%\%\%\%\%\%\%\%\%\%\%\%\%\%\%\%\%\%\%\%\%\%\%\%\%\%\%\%\%\%\%\%\%

$\%$ Wes Burwash

$\%$ Carleton University BVI PIV

$\%$ Rotorcraft Group

$\%$ October 5, 2006

$\% \quad$ PART OF: PIV Post Processing Tools

$\%$ Function Description:

$\%$ This function plots the vector map 'vector_map' which was created by

$\%$ read_dat.m. It also employed the vector thinning function thin.m which

$\%$ required a factor by which to reduce the number of vectors displyed. The

$\% \quad$ title to be displayed is entered as 'plot_title'.

$\% \% \% \% \% \% \% \% \% \% \% \% \% \% \% \% \% \% \% \% \% \% \% \% \% \% \% \% \% \% \% \% \% \% \% \% \% \% \% \% \% \% \% \% \% \% \% \% \% \% \% \% \% \% \% \% \% \% \% \% \% \% \% \% \% \% \%$

vector_map_thin $=$ thin (vector_map, factor);

quiver (vector_map_thin $(1,:,:)$, vector_map_thin $(2,:,:)$, vector_map_thin $(3,:,:)$, vector_map_thin $(4,:,:), 0.6, ' k$ ') ; title(plot_title);

xlabel('Distance from tail (mm)');

ylabel('Distance from chord(mm)');

$\%$ axis square;

axis equal;

axis tight;

\section{Plot Absolute Velocity}

function absplot(vector_map, plot_title)

$\% \% \% \% \% \% \% \% \% \% \% \% \% \% \% \% \% \% \% \% \% \% \% \% \% \% \% \% \% \% \% \% \% \% \% \% \% \% \% \% \% \% \% \% \% \% \% \% \% \% \% \% \% \% \% \% \% \% \% \% \% \% \% \% \% \% \% \% \% \% \% \% \% \%$

$\%$ Wes Burwash

$\% \quad$ Carleton University BVI PIV 


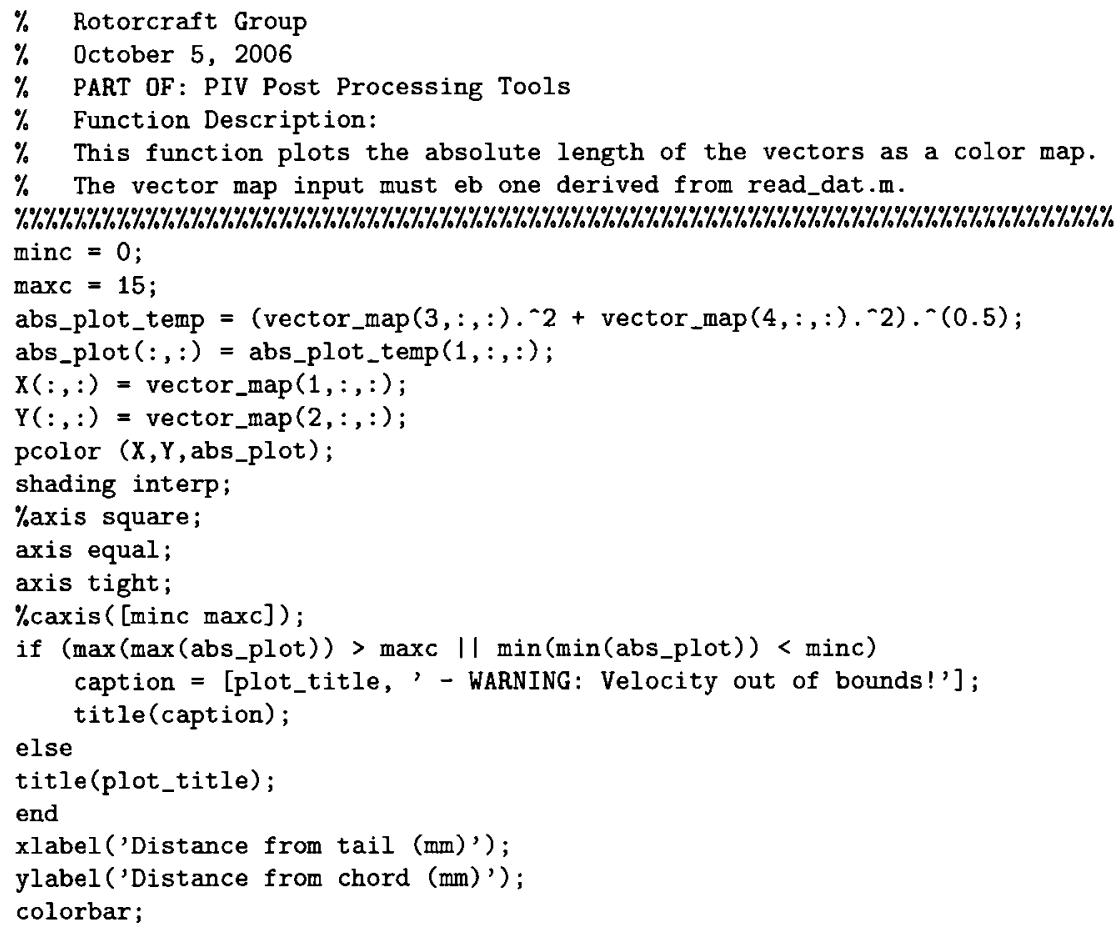


Plot Velocity Vectors withe Absolute Velocity Painted on Background

function vel_abs_plot (vectors, factor)

\%\%\%\%\%\%\%\%\%\%\%\%\%\%\%\%\%\%\%\%\%\%\%\%\%\%\%\%\%\%\%\%\%\%\%\%\%\%\%\%\%\%\%\%\%\%\%\%\%\%\%\%\%\%\%\%\%\%\%\%\%\%\%\%\%\%\%\%\%\%\%\%\%\%\%\%

$\%$ Wes Burwash

$\%$ Carleton University BVI PIV

$\%$ Rotorcraft Group

$\%$ October 5, 2006

$\% \quad$ PART OF: PIV Post Processing Tools

$\% \quad$ Function Description:

$\%$ This function plots the velocity vectors with the background as the

$\%$ absolute value of the velocities.

$\% \% \% \% \% \% \% \% \% \% \% \% \% \% \% \% \% \% \% \% \% \% \% \% \% \% \% \% \% \% \% \% \% \% \% \% \% \% \% \% \% \% \% \% \% \% \% \% \% \% \% \% \% \% \% \% \% \% \% \% \% \% \% ~$

vecplot (vectors, factor, 'Velocity (m/s)');

hold on;

absplot (vectors, 'Velocity (m/s)');

hold off;

\section{Subtract on Velocity Vector Field from Another}

function diff = subvecs $(A, B)$

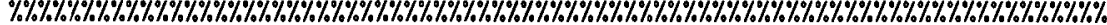

$\%$ Wes Burwash

$\%$ Carleton University BVI PIV

$\%$ Rotorcraft Group

$\%$ October 5, 2006

$\% \quad$ PART DF: PIV Post Processing Tools

$\%$ Function Description:

$\% \quad$ This function subtracts the $U$ an $V$ components of $B$ from $A$. A and B are

$\%$ both vector plots creaded by read_dat.m.

$\%$ I.E. A - B

\%\%\%\%\%\%\%\%\%\%\%\%\%\%\%\%\%\%\%\%\%\%\%\%\%\%\%\%\%\%\%\%\%\%\%\%\%\%\%\%\%\%\%\%\%\%\%\%\%\%\%\%\%\%\%\%\%\%\%\%\%\%\%\%\%\%\%\%\%\%\%\%\%\%\%\%\%\%

$\operatorname{diff}(1: 2,:,:)=A(1: 2,:,:)$

$\operatorname{diff}(3: 4,:,:)=A(3: 4,:,:)-B(3: 4,:,:)$; 


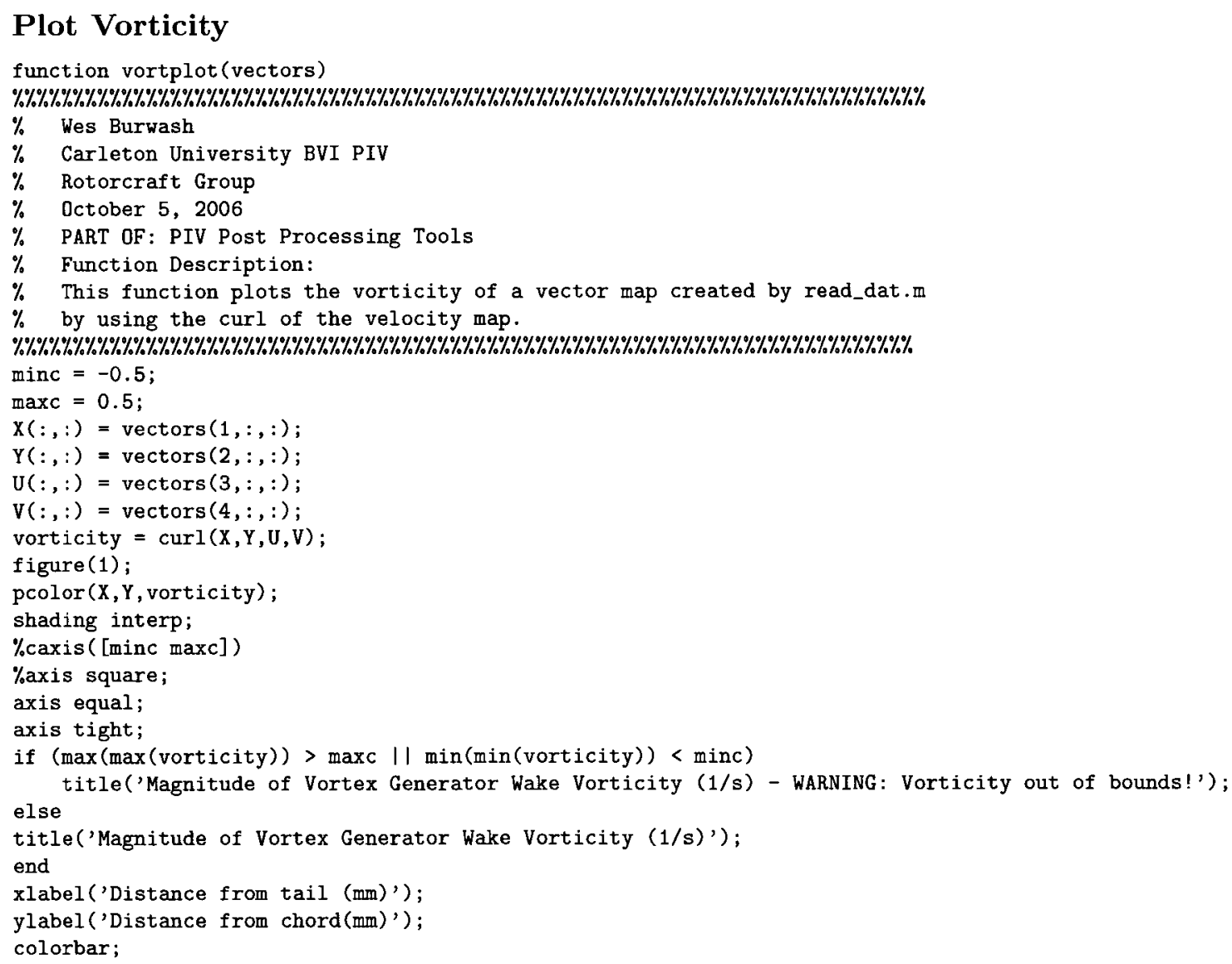

\section{Plot Velocity Vectors withe Vorticity Painted on Background}

function vel_vort_plot(vectors, factor) $\% \% \% \% \% \% \% \% \% \% \% \% \% \% \% \% \% \% \% \% \% \% \% \% \% \% \% \% \% \% \% \% \% \% \% \% \% \% \% \% \% \% \% \% \% \% \% \% \% \% \% \% \% \% \% \% \% \% \% \% \% \% \% \% \% \%$

$\%$ Wes Burwash

$\% \quad$ Carleton University BVI PIV

$\% \quad$ Rotorcraft Group

$\%$ October 5,2006

$\% \quad$ PART OF: PIV Post Processing Tools

$\% \quad$ Function Description:

$\%$ This function plots the velocity vectors with the background set as the $\%$ vorticity.

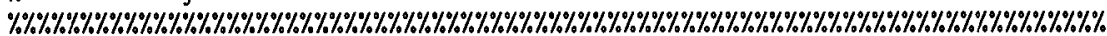

figure (1)

vortplot(vectors)

hold on;

vecplot (vectors (:, :, 1:59), factor, 'Vorticity (1/s) with Velocity Vectors');

hold of $\mathrm{f}$ 


\section{Calculate Dynamic Pressure}

function press = dpress (vects, fanspd) \%\%\%\%\%\%\%\%\%\%\%\%\%\%\%\%\%\%\%\%\%\%\%\%\%\%\%\%\%\%\%\%\%\%\%\%\%\%\%\%\%\%\%\%\%\%\%\%\%\%\%\%\%\%\%\%\%\%\%\%\%\%\%\%\%\%\%\%\%\%\%\%\%\%\%\%\%

$\%$ Wes Burwash

$\%$ Carleton University BVI PIV

$\% \quad$ Rotorcraft Group

$\%$ October 5, 2006

$\% \quad$ PART OF: PIV Post Processing Tools

$\%$ Function Description:

$\%$ This function takes in a vector map of velociteis and converts it to a

$\%$ map of dynamic pressure using the relation $p=$ rho*vel $-2 / 2$. The input

$\%$ 'fanspd' is used to calculate the air density from the speed of the fan

$\% \quad$ in $\mathrm{Hz}$. TH efunction returns the dynamic pressure filed in 'press'.

$\% \% \% \% \% \% \% \% \% \% \% \% \% \% \% \% \% \% \% \% \% \% \% \% \% \% \% \% \% \% \% \% \% \% \% \% \% \% \% \% \% \% \% \% \% \% \% \% \% \% \% \% \% \% \% \% \% \%$

temp $=0.0031 *$ fanspd ${ }^{\wedge}+0.4675 *$ fanspd +292.22 ;

rho $=360.77819 *$ temp ${ }^{-}(-1.00336)$;

$\operatorname{press}(1: 2,:,:)=\operatorname{vects}(1: 2,:,:) ;$

$\operatorname{press}(3: 4,:,:)=((\operatorname{vects}(3: 4,:,:)) \cdot-2) * \operatorname{rho} / 2$;

Plot Velocity Vectors withe Dynamic Pressure Painted on Background

function ve1_press_plot (vectors, factor, fanspd) \%\%\%\%\%\%\%\%\%\%\%\%\%\%\%\%\%\%\%\%\%\%\%\%\%\%\%\%\%\%\%\%\%\%\%\%\%\%\%\%\%\%\%\%\%\%\%\%\%\%\%\%\%\%\%\%\%\%\%\%\%\%\%\%\%\%\%\%\%\%

$\%$ Wes Burwash

$\%$ Carleton University BVI PIV

$\%$ Rotorcraft Group

$\%$ October 5, 2006

$\% \quad$ PART OF: PIV Post Processing Tools

$\%$ Function Description:

$\%$ This function plots the velocity vectors with the background set as the $\%$ dynamic pressure.

$\% \% \% \% \% \% \% \% \% \% \% \% \% \% \% \% \% \% \% \% \% \% \% \% \% \% \% \% \% \% \% \% \% \% \% \% \% \% \% \% \% \% \% \% \% \% \% \% \% \% \% \% \% \% \% \% \% \% \% ~$

pressure $=\operatorname{dpress}($ vectors, fanspd);

figure (1)

absplot (pressure, 'Pressure (kg/(m*s²)) with Velocity Vectors');

hold on;

vecplot (vectors, factor, 'Pressure $\left(\mathrm{kg} /\left(\mathrm{m}^{*} \mathrm{~s}^{\wedge} 2\right)\right)$ with Velocity Vectors');

hold off; 


\section{Calculate Turbulence}

function turbulence $=$ turb (base_name, startn, endn)

\%\%\%\%\%\%\%\%\%\%\%\%\%\%\%\%\%\%\%\%\%\%\%\%\%\%\%\%\%\%\%\%\%\%\%\%\%\%\%\%\%\%\%\%\%\%\%\%\%\%\%\%\%\%\%\%\%\%\%\%\%\%\%\%\%\%\%\%\%\%\%\%\%\%

$\%$ Wes Burwash

$\% \quad$ Carleton University BVI PIV

$\% \quad$ Rotorcraft Group

$\% \quad$ October 5,2006

$\% \quad$ PART OF: PIV Post Processing Tools

$\% \quad$ Function Description:

$\%$ This function estimates the amount of tubulence present in the flow.

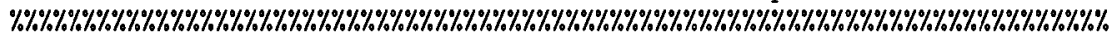
avg = average (base_name, startn, endn);

ext='txt';

out $=0$;

for ( $i=$ startn:endn)

num $=$ num2str $(i)$;

if $(i<10)$ end

file_name $=$ [base_name, '000', num, '.', ext] :

if $(i<100 \& \& i>=10)$

end

file_name $=\left[\right.$ base_name, ' $00^{\prime}$, num, ' .', ext];

end

turbulence $=$ turbulence $+\left(\right.$ subvecs $\left(\right.$ read_dat $\left.\left.\left(f i l e \_n a m e\right), a v g\right)\right) . \wedge 2$;

turbulence $=$ turbulence $/($ endn-startn+1);

\section{Flip Vector Field about Horizontal}

function flipped = flip_horiz(orig)

\%\%\%\%\%\%\%\%\%\%\%\%\%\%\%\%\%\%\%\%\%\%\%\%\%\%\%\%\%\%\%\%\%\%\%\%\%\%\%\%\%\%\%\%\%\%\%\%\%\%\%\%\%\%\%\%\%\%\%\%\%\%\%\%\%\%\%\%\%\%\%\%

$\%$ Wes Burwash

\% Carleton University BVI PIV

$\% \quad$ Rotorcraft Group

$\%$ October 5, 2006

PART OF: PIV Post Processing Tools

Function Description:

$\%$ This function takes in a vector map of velociteis and flips it about

$\%$ the horizontal axis.

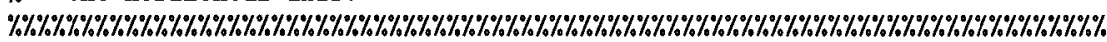

flipped $(1: 2,:,:)=\operatorname{orig}(1: 2,:,:)$;

[four, cols, rows] = size(orig);

for ( $i=1$ :rows)

end

flipped $(3: 4,:, i)=\operatorname{orig}(3: 4,:$, rows $-i+1) ;$

flipped $(3,:,:)=-f l i p p e d(3,:,:) ;$ 


\section{Flip Vector Field about Vertical}

function flipped $=$ flip_vert (orig)

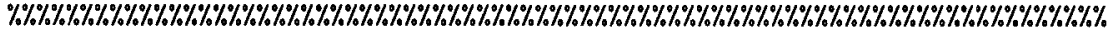

$\%$ Wes Burwash

$\%$ Carleton University BVI PIV

$\%$ Rotorcraft Group

$\% \quad$ October 5, 2006

$\% \quad$ PART OF: PIV Post Processing Tools

$\%$ Function Description:

$\%$ This function takes in a vector map of velociteis and flips it about

$\%$ the vertical axis.

$\% \% \% \% \% \% \% \% \% \% \% \% \% \% \% \% \% \% \% \% \% \% \% \% \% \% \% \% \% \% \% \% \% \% \% \% \% \% \% \% \% \% \% \% \% \% \% \% \% \% \% \% \% \% \% \% \% \% \% \% \% \% \% \% \% \%$

flipped $(1: 2,:,:)=\operatorname{orig}(1: 2,:,:)$;

[four, cols, rows] = size(orig);

for ( $i=1$ : cols)

end

flipped $(3: 4, i,:)=\operatorname{orig}(3: 4, \operatorname{col} s-i+1,:) ;$

flipped $(4,:,:)=-f l i p p e d(4,:,:)$; 